\title{
N-DETACHABLE PAIRS IN 3-CONNECTED MATROIDS III: THE THEOREM
}

\author{
NICK BRETTELL, GEOFF WHITTLE, AND ALAN WILLIAMS
}

\begin{abstract}
Let $M$ be a 3-connected matroid, and let $N$ be a 3 connected minor of $M$. A pair $\left\{x_{1}, x_{2}\right\} \subseteq E(M)$ is $N$-detachable if one of the matroids $M / x_{1} / x_{2}$ or $M \backslash x_{1} \backslash x_{2}$ is 3 -connected and has an $N$-minor. This is the third and final paper in a series where we prove that if $|E(M)|-|E(N)| \geq 10$, then either $M$ has an $N$-detachable pair after possibly performing a single $\Delta-Y$ or $Y-\Delta$ exchange, or $M$ is essentially $N$ with a spike attached. Moreover, we describe the additional structures that arise if we require only that $|E(M)|-|E(N)| \geq 5$.
\end{abstract}

\section{INTRODUCTION}

Let $M$ be a 3-connected matroid, and let $N$ be a 3-connected minor of $M$. We say that a matroid has an $N$-minor if it has an isomorphic copy of $N$ as a minor. A pair $\left\{x_{1}, x_{2}\right\} \subseteq E(M)$ is $N$-detachable if either

(a) $M / x_{1} / x_{2}$ is 3 -connected and has an $N$-minor, or

(b) $M \backslash x_{1} \backslash x_{2}$ is 3 -connected and has an $N$-minor.

This is the third paper in a series [3, 4] where we describe the structures that arise when it is not possible to find an $N$-detachable pair in $M$. We prove the following theorem:

Theorem 1.1. Let $M$ be a 3-connected matroid with $|E(M)| \geq 13$, and let $N$ be a 3-connected minor of $M$ such that $|E(N)| \geq 4$, and $|E(M)|-$ $|E(N)| \geq 5$. Then either

(i) $M$ has an $N$-detachable pair,

(ii) there is a matroid $M^{\prime}$ obtained by performing a single $\Delta-Y$ or $Y-\Delta$ exchange on $M$ such that $M^{\prime}$ has an $N$-detachable pair, or

(iii) there exists $P \subseteq E(M)$ such that at most one element of $E(M)-$ $E(N)$ is not in $P$, and $P$ is either

(a) an augmented quad 3-separator, wherein $|E(M)|-|E(N)|=5$,

(b) a twisted-cube-like 3-separator of $M$ or $M^{*}$, in which case $|E(M)|-|E(N)|=5$

(c) a skew-whiff 3-separator, in which case $|E(M)|-|E(N)| \leq 7$,

Date: February 3, 2021.

2020 Mathematics Subject Classification. 05B35.

Key words and phrases. 3-connected matroid, Splitter Theorem, simple 3-connected graph, Wheels and Whirls Theorem.

The authors were supported by the New Zealand Marsden Fund. 
(d) an elongated-quad 3-separator, wherein $|E(M)|-|E(N)| \leq 7$,

(e) a double-quad 3-separator, in which case $|E(M)|-|E(N)| \leq 9$, or

(f) a spike-like 3 -separator.

The 3-separators that appear in Theorem 1.1(iii) are defined momentarily, while $\Delta-Y$ and $Y-\Delta$ exchange are defined in Section 2 ,

By restricting attention to the case where $|E(M)|-|E(N)| \geq 10$, Theorem 1.1 implies the theorem stated in the first paper of the series [3, Theorem 1.1]. The motivation for proving such a theorem comes from a desire to find exact excluded-minor characterisations of certain minor-closed classes of representable matroids (for more details, see the introduction to [3]).

The structure of this paper is as follows. In Section 2, we review relevant preliminaries regarding connectivity and keeping an $N$-minor, and results from the first two papers in the series. In Section 3 we lay the ground work towards a proof of Theorem 1.1. The main hurdle still to overcome is addressed in Section 4, there, we consider the case where every pair whose deletion or contraction keeps an $N$-minor results in a non-trivial series or parallel class, respectively. Throughout the first two papers, we have built up a collection of particular 3-separators that can appear in a matroid with no $N$-detachable pairs. In Section 5 , we show that when $M$ has one of these particular 3-separators, $P$, and no $N$-detachable pairs, then at most one element of $M$ is not in $E(N) \cup P$. Finally, we put everything together in Section 6 to prove Theorem 6.2, which implies Theorem 1.1 .

We close this series of papers with some final remarks in Section 7 . There, we address the natural question of what additional structures might arise if we do not allow the single $\Delta-Y$ or $Y-\Delta$ exchange. We also present an analogue of Theorem 1.1 for graphic matroids, and discuss the implications for simple 3-connected graphs.

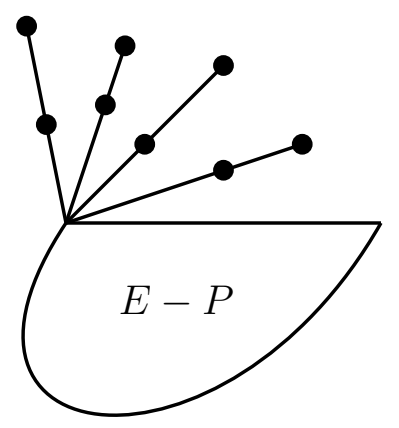

Figure 1. An example of a spike-like 3-separator $P$, which can appear in a matroid $M$ that does not contain any $N$ detachable pairs, even when $|E(M)|-|E(N)|>9$. 
Particular 3-separators. Let $M$ be a matroid with ground set $E$. We say that a 4-element set $Q \subseteq E$ is a quad if it is both a circuit and a cocircuit of $M$. We can also view $Q$ as a particular 3-separator that can give rise to matroids with no $N$-detachable pairs. For this reason, we also sometimes refer to $Q$ as a quad 3-separator.

Definition 1.1. Let $P \subseteq E$ be a $2 t$-element, rank- $(t+1)$, corank- $(t+1)$, exactly 3 -separating set of $M$, for some $t \geq 3$. If there exists a partition $\left\{L_{1}, \ldots, L_{t}\right\}$ of $P$ such that

(a) $\left|L_{i}\right|=2$ for each $i \in\{1,2, \ldots, t\}$, and

(b) $L_{i} \cup L_{j}$ is a quad for all distinct $i, j \in\{1,2, \ldots, t\}$,

then $P$ is a spike-like 3 -separator of $M$. For each $i \in\{1, \ldots, t\}$, we say $L_{i}$ is a leg of $P$.

Definition 1.2. Let $P \subseteq E$ be a 6-element, rank-4, corank-4, exactly 3separating set of $M$. If there exists a labelling $\left\{s_{1}, s_{2}, t_{1}, t_{2}, u_{1}, u_{2}\right\}$ of $P$ such that

(a) $\left\{s_{1}, s_{2}, t_{2}, u_{1}\right\},\left\{s_{1}, t_{1}, t_{2}, u_{2}\right\}$, and $\left\{s_{2}, t_{1}, u_{1}, u_{2}\right\}$ are the circuits of $M$ contained in $P$, and

(b) $\left\{s_{1}, s_{2}, t_{1}, t_{2}\right\},\left\{s_{1}, s_{2}, u_{1}, u_{2}\right\}$, and $\left\{t_{1}, t_{2}, u_{1}, u_{2}\right\}$ are the cocircuits of $M$ contained in $P$,

then $P$ is a skew-whiff 3-separator of $M$.

Definition 1.3. Let $P \subseteq E$ be a 6-element, rank-4, corank-4, exactly 3separating set such that $P=Q \cup\left\{p_{1}, p_{2}\right\}$ and $Q$ is a quad. If there exists a labelling $\left\{q_{1}, q_{2}, q_{3}, q_{4}\right\}$ of $Q$ such that

(a) $\left\{p_{1}, p_{2}, q_{1}, q_{2}\right\},\left\{p_{1}, p_{2}, q_{3}, q_{4}\right\}$, and $Q$ are the circuits of $M$ contained in $P$, and

(b) $\left\{p_{1}, p_{2}, q_{1}, q_{3}\right\},\left\{p_{1}, p_{2}, q_{2}, q_{4}\right\}$, and $Q$ are the cocircuits of $M$ contained in $P$,

then $P$ is an elongated-quad 3-separator of $M$.

Definition 1.4. Let $P \subseteq E$ be a rank-5, corank-5, exactly 3-separating set such that $P=Q_{1} \cup Q_{2}$ where $Q_{1}$ and $Q_{2}$ are disjoint quads of $M$. If there exist labellings $\left\{p_{1}, p_{2}, p_{3}, p_{4}\right\}$ of $Q_{1}$, and $\left\{q_{1}, q_{2}, q_{3}, q_{4}\right\}$ of $Q_{2}$ such that

(a) $\left\{p_{1}, p_{2}, q_{1}, q_{2}\right\},\left\{p_{1}, p_{2}, q_{3}, q_{4}\right\},\left\{p_{3}, p_{4}, q_{1}, q_{2}\right\},\left\{p_{3}, p_{4}, q_{3}, q_{4}\right\}, Q_{1}$, and $Q_{2}$ are the circuits of $M$ contained in $P$, and

(b) $\left\{p_{1}, p_{3}, q_{1}, q_{3}\right\},\left\{p_{1}, p_{3}, q_{2}, q_{4}\right\},\left\{p_{2}, p_{4}, q_{1}, q_{3}\right\},\left\{p_{2}, p_{4}, q_{2}, q_{4}\right\}, Q_{1}$, and $Q_{2}$ are the cocircuits of $M$ contained in $P$,

then $P$ is a double-quad 3 -separator with associated partition $\left\{Q_{1}, Q_{2}\right\}$.

Definition 1.5. Let $P \subseteq E$ be a rank-4, corank-4, exactly 3-separating set with $P=\left\{p_{1}, p_{2}, q_{1}, q_{2}, s_{1}, s_{2}\right\}$. Suppose that

(a) $\left\{p_{1}, p_{2}, s_{1}, s_{2}\right\},\left\{q_{1}, q_{2}, s_{1}, s_{2}\right\}$, and $\left\{p_{1}, p_{2}, q_{1}, q_{2}\right\}$ are the circuits of $M$ contained in $P$, and 
(b) $\left\{p_{1}, q_{1}, s_{1}, s_{2}\right\}, \quad\left\{p_{2}, q_{2}, s_{1}, s_{2}\right\}, \quad\left\{p_{1}, p_{2}, q_{1}, q_{2}, s_{1}\right\}, \quad$ and $\left\{p_{1}, p_{2}, q_{1}, q_{2}, s_{2}\right\}$ are the cocircuits of $M$ contained in $P$.

Then $P$ is a twisted-cube-like 3-separator of $M$.

Let $M$ be a 3-connected matroid with a 3-connected minor $N$ such that $|E(M)|-|E(N)| \geq 5$, and $M$ has no $N$-detachable pairs. For any particular

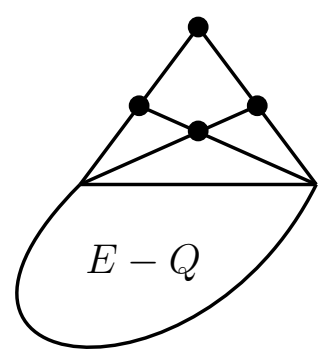

(a) A quad 3-separator $Q$.

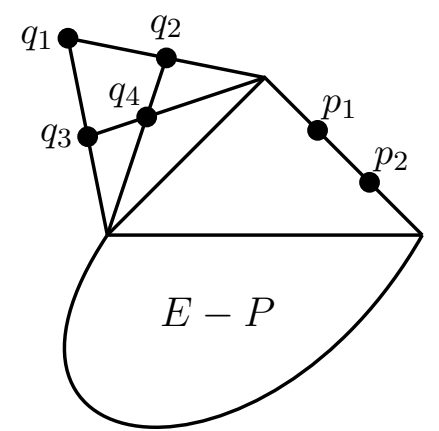

(c) An elongated-quad 3-separator.

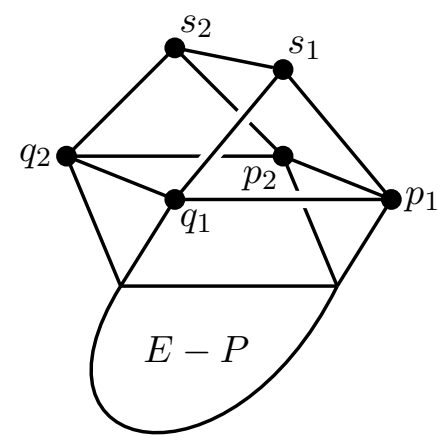

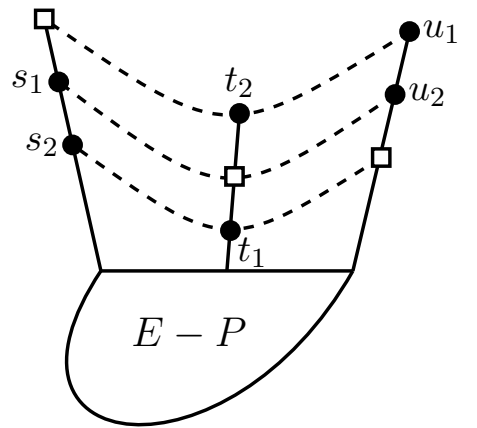

(b) A skew-whiff 3-separator.

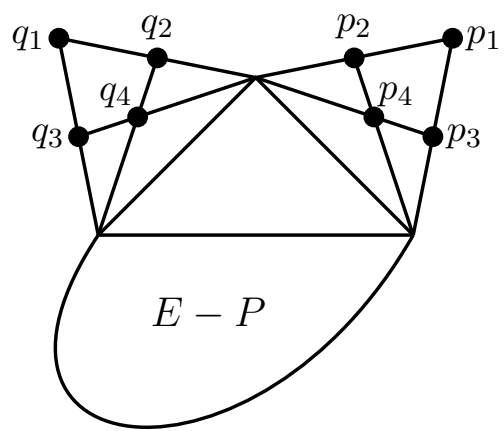

(d) A double-quad 3-separator.

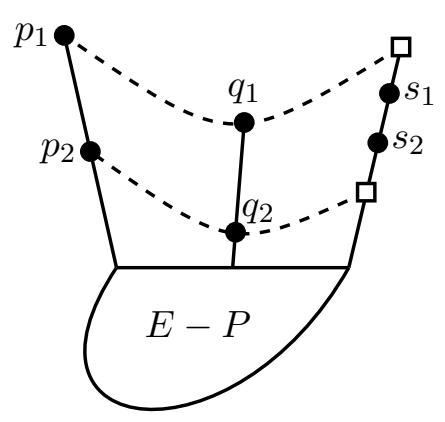

(e) A twisted-cube-like 3-separator of $M$. (f) A twisted-cube-like 3-separator of $M^{*}$.

Figure 2. A quad 3-separator $Q$, and each remaining particular 3-separator $P$ that can appear in a matroid $M$ with no $N$-detachable pairs, where $|E(M)|-|E(N)| \geq 5$ 
3-separator $P$ as just defined, $P$ can appear in $M$, and have the property that at most one element of $M$ is not in $E(N) \cup P$. Examples of such matroids with $E(M)=E(N) \cup P$ were given in [3, 4. There also exist such $M, N$, and $P$ where a single element of $E(M)$ is not in $E(N) \cup P$. Before giving an example, we require the following definitions.

Let $P$ be a particular 3 -separator. If there exists some $z \in E(M)-P$ such that $P \cup z$ is exactly 3 -separating, then we say that $P \cup z$ is an augmented 3-separator. In this case, either $z \in \operatorname{cl}(P)-P$ or $z \in \mathrm{cl}^{*}(P)-P$ (due to the soon-to-appear Lemma 2.4). We also say that an augmented 3-separator $P \cup$ $z$ is an augmentation of $P$.

When $M$ has no $N$-detachable pairs and contains an augmented 3separator $P \cup z$, it is possible that $z$, when removed in the correct way, preserves the $N$-minor. A careful reader might have wondered why we have included the quad as a particular 3-separator, given that we are only interested in the case where $|E(M)|-|E(N)| \geq 5$. The reason is that an augmented quad 3-separator $Q \cup z$ can appear in a matroid with no $N$ detachable pairs, where $E(M)=E(N) \cup(Q \cup z)$, in which case it is possible that $|E(M)|-|E(N)|=5$. We now give an example of such a matroid $M$, where $N$ is the Fano matroid.

Let $F_{7}$ be a copy of the Fano matroid with a triangle $\{a, b, c\}$, and let $F_{7}^{-}$be a copy of the non-Fano matroid with a triangle $\{x, y, z\}$, where the ground sets of $F_{7}$ and $F_{7}^{-}$are disjoint. Let $Q=E\left(F_{7}^{-}\right)-\{x, y, z\}$. Let $\left(F_{7}^{-}\right)^{\prime}$ be the matroid obtained by freely adding the element $a$ on the line $\{x, y, z\}$. Let $M^{\prime}=\left(F_{7}^{-}\right)^{\prime} \oplus_{2} F_{7}$, where $a$ is the basepoint of this 2-sum. Finally, we obtain $M$ by freely adding an element $h$ on the line $\{x, c\}$, then deleting $\{x, y, z\}$. Note that $M$ is 3-connected, $Q \cup h$ is an augmented quad 3 -separator of $M$ with $h \in \mathrm{cl}^{*}(Q)$, and $M$ has no $F_{7}$-detachable pairs. In particular, $M \backslash h$ has an $F_{7}$-minor, but $M / h$ does not.

In a similar manner, one can obtain augmentations of the other particular 3 -separators, except the twisted-cube-like 3-separator. For a twisted-cubelike 3-separator $P$, labelled as in Figure 2(e) say, the existence of an element $e \in \operatorname{cl}(P)-P$ for which $M / e$ has an $N$-minor ensures that a detachable pair like $\left\{q_{1}, p_{2}\right\}$ or $\left\{q_{2}, p_{1}\right\}$ is also $N$-detachable (and similarly for $e \in$ $\left.\mathrm{cl}^{*}(P)-P\right)$.

\section{Preliminaries}

The notation and terminology in the paper follow Oxley [6]. For a set $X$ and element $e$, we write $X \cup e$ instead of $X \cup\{e\}$, and $X-e$ instead of $X-\{e\}$. We say that $X$ meets $Y$ if $X \cap Y \neq \emptyset$. We denote $\{1,2, \ldots, n\}$ by $[n]$. The phrase "by orthogonality" refers to the fact that a circuit and a cocircuit cannot intersect in exactly one element.

Let $M$ be a matroid. For $P \subseteq E(M)$, we say that $P$ is a 5 -element plane if $M \mid P \cong U_{3,5}$. We also say $P$ is a 5-element coplane if $M^{*} \mid P \cong U_{3,5}$. The 
following two lemmas are straightforward consequences of orthogonality, and are used freely without reference.

Lemma 2.1. Suppose $M$ is a matroid with a 5-element plane $P$. If $C^{*}$ is a cocircuit of $M$ that meets $P$, then $\left|P \cap C^{*}\right| \geq 3$.

Lemma 2.2. Let $(X,\{e\}, Y)$ be a partition of the ground set of a matroid $M$. Then $e \in \operatorname{cl}(X)$ if and only if $e \notin \mathrm{cl}^{*}(Y)$.

Connectivity. Let $M$ be a matroid with ground set $E$. The connectivity function of $M$, denoted by $\lambda_{M}$, is defined as follows, for all subsets $X$ of $E$ :

$$
\lambda_{M}(X)=r(X)+r(E-X)-r(M) .
$$

A subset $X$ or a partition $(X, E-X)$ of $E$ is $k$-separating if $\lambda_{M}(X) \leq$ $k-1$. A $k$-separating partition $(X, E-X)$ is a $k$-separation if $|X| \geq k$ and $|E-X| \geq k$. A $k$-separating set $X$, or a $k$-separating partition $(X, E-X)$, is exact if $\lambda_{M}(X)=k-1$. A matroid is $n$-connected if it has no $k$-separations for all $k<n$. When a matroid is 2-connected, we simply say it is connected.

The connectivity functions of a matroid and its dual are equal; that is, $\lambda_{M}(X)=\lambda_{M^{*}}(X)$. In fact, it is easily shown that

$$
\lambda_{M}(X)=r(X)+r^{*}(X)-|X| .
$$

The next lemma is a consequence of the fact that the connectivity function is submodular. We write "by uncrossing" to refer to an application of this lemma.

Lemma 2.3. Let $M$ be a 3-connected matroid, and let $X$ and $Y$ be 3separating subsets of $E$.

(i) If $|X \cap Y| \geq 2$, then $X \cup Y$ is 3-separating.

(ii) If $|E-(X \cup Y)| \geq 2$, then $X \cap Y$ is 3-separating.

The following is well known.

Lemma 2.4. Let $M$ be a 3-connected matroid, and let $(X,\{e\}, Y)$ be a partition of $E$ such that $X$ is exactly 3-separating. Then

(i) $X \cup e$ is 3-separating if and only if $e \in \operatorname{cl}(X)$ or $e \in \mathrm{cl}^{*}(X)$, and

(ii) $X \cup e$ is exactly 3-separating if and only if either $e \in \operatorname{cl}(X) \cap \operatorname{cl}(Y)$ or $e \in \mathrm{cl}^{*}(X) \cap \mathrm{cl}^{*}(Y)$.

When Lemma 2.4(ii) holds, we say $e$ is a guts element if $e \in \operatorname{cl}(X) \cap \operatorname{cl}(Y)$, and $e$ is a coguts element if $e \in \mathrm{cl}^{*}(X) \cap \mathrm{cl}^{*}(Y)$.

Lemma 2.5. Let $M$ be a 3-connected matroid, let $(X, E-X)$ be a 3separation of $M$, and let $e \in E-X$. Then $e \notin \operatorname{cl}(X) \cap \mathrm{cl}^{*}(X)$.

A 3-separation $(X, E-X)$ of a matroid $M$ with ground set $E$ is vertical if $r(X) \geq 3$ and $r(E-X) \geq 3$. We also say a partition $(X,\{z\}, Y)$ of $E$ is a vertical 3-separation when $(X \cup z, Y)$ and $(X, Y \cup z)$ are both vertical 3-separations and $z \in \operatorname{cl}(X) \cap \operatorname{cl}(Y)$. 
A vertical 3-separation in $M^{*}$ is known as a cyclic 3 -separation in $M$. More specifically, a 3-separation $(X, E-X)$ of $M$ is cyclic if $r^{*}(X) \geq 3$ and $r^{*}(E-X) \geq 3$; or, equivalently, if $X$ and $E-X$ contain circuits. We also say that a partition $(X,\{z\}, Y)$ of $E$ is a cyclic 3 -separation if $(X,\{z\}, Y)$ is a vertical 3 -separation in $M^{*}$.

Lemma 2.6 ([11, Lemma 3.5]). Let $M$ be a 3-connected matroid and let $z \in E$. The following are equivalent:

(i) $M$ has a vertical 3 -separation $(X,\{z\}, Y)$.

(ii) $\operatorname{si}(M / z)$ is not 3 -connected.

We say that a partition $\left(X_{1}, X_{2}, \ldots, X_{m}\right)$ of $E$ is a path of $k$-separations if $\left(X_{1} \cup \cdots \cup X_{i}, X_{i+1} \cup \cdots \cup X_{m}\right)$ is a $k$-separation for each $i \in[m-1]$. Observe that a vertical, or cyclic, 3-separation $(X,\{z\}, Y)$ is an instance of a path of 3-separations.

We refer to the following as Bixby's Lemma.

Lemma 2.7 (Bixby's Lemma [1]). Let e be an element of a 3-connected matroid $M$. Then either $\operatorname{si}(M / e)$ is 3-connected, or $\operatorname{co}(M \backslash e)$ is 3-connected.

The next lemma [11] shows, in particular, that an element that is in a quad but not in a triangle (or a triad) can be contracted (or deleted, respectively) without destroying 3-connectivity.

Lemma 2.8. Let $C^{*}$ be a rank-3 cocircuit of a 3-connected matroid $M$. If $x \in C^{*}$ has the property that $\mathrm{cl}_{M}\left(C^{*}\right)-x$ contains a triangle of $M / x$, then $\operatorname{si}(M / x)$ is 3-connected.

If, rather than a quad, we have a 4-element independent cocircuit, then the following lemma applies. The proof of this lemma is similar to [3, Lemma 4.5]; we omit the details.

Lemma 2.9. Let $M$ be a 3 -connected matroid and let $C^{*}=\left\{c_{1}, c_{2}, d_{1}, d_{2}\right\}$ be a 4-element cocircuit such that $M \backslash d_{1}$ and $M \backslash d_{2}$ are 3 -connected, and neither $c_{1}$ nor $c_{2}$ is in a triangle. Then $M / c_{i}$ is 3-connected for some $i \in\{1,2\}$.

We also require the following result, proved in 3 .

Lemma 2.10 ([3, Lemma 4.4]). Let $M$ be a 3-connected matroid with $P \subseteq E$ such that $M \mid P \cong U_{3,6}$, and $X \subseteq P$ such that $|X|=4$. Suppose that $\operatorname{cl}(P)$ contains no triangles. Then there are distinct elements $x_{1}, x_{2} \in X$ such that $M \backslash x_{1} \backslash x_{2}$ is 3-connected.

\section{Retaining an $N$-minor.}

Lemma 2.11. Let $(S, T)$ be a 2-separation of a connected matroid $M$ and let $N$ be a 3-connected minor of $M$. Then $\{S, T\}$ has a member $U$ such that $|U \cap E(N)| \leq 1$. Moreover, if $u \in U$, then

(i) $M / u$ has an $N$-minor if $M / u$ is connected, and

(ii) $M \backslash u$ has an $N$-minor if $M \backslash u$ is connected. 
For a matroid $M$ with a minor $N$ and $e \in E(M)$, we say $e$ is $N$-contractible if $M / e$ has an $N$-minor, we say $e$ is $N$-deletable if $M \backslash e$ has an $N$-minor, and we say $e$ is $N$-flexible if $e$ is both $N$-contractible and $N$-deletable.

The dual of the following is proved in [2, 5].

Lemma 2.12. Let $N$ be a 3-connected minor of a 3-connected matroid $M$. Let $(X,\{z\}, Y)$ be a cyclic 3 -separation of $M$ such that $M \backslash z$ has an $N$-minor with $|X \cap E(N)| \leq 1$. Let $X^{\prime}=X-\operatorname{cl}^{*}(Y)$ and $Y^{\prime}=\mathrm{cl}^{*}(Y)-z$. Then

(i) each element of $X^{\prime}$ is $N$-deletable; and

(ii) at most one element of $\mathrm{cl}^{*}(X)-z$ is not $N$-contractible, and if such an element $x$ exists, then $x \in X^{\prime} \cap \operatorname{cl}\left(Y^{\prime}\right)$ and $z \in \mathrm{cl}^{*}\left(X^{\prime}-x\right)$.

Suppose $C$ and $D$ are disjoint subsets of $E(M)$ such that $M / C \backslash D \cong N$. We call the ordered pair $(C, D)$ an $N$-labelling of $M$, and say that each $c \in C$ is $N$-labelled for contraction, and each $d \in D$ is $N$-labelled for deletion. We also say a set $C^{\prime} \subseteq C$ is $N$-labelled for contraction, and $D^{\prime} \subseteq D$ is $N$-labelled for deletion. An element $e \in C \cup D$ or a set $X \subseteq C \cup D$ is $N$-labelled for removal.

Let $(C, D)$ be an $N$-labelling of $M$, and let $c \in C, d \in D$, and $e \in$ $E(M)-(C \cup D)$. Then, we say that the ordered pair $((C-c) \cup d,(D-d) \cup c)$ is obtained from $(C, D)$ by switching the $N$-labels on $c$ and $d$. Similarly, $((C-c) \cup e, D)$ (or $(C,(D-d) \cup e)$, respectively) is obtained from $(C, D)$ by switching the $N$-labels on $c$ (respectively, $d$ ) and $e$.

The following straightforward lemma, which gives a sufficient condition for retaining a valid $N$-labelling after an $N$-label switch, will be used freely.

Lemma 2.13. Let $M$ be a 3-connected matroid, let $N$ be a 3-connected minor of $M$ with $|E(N)| \geq 4$, and let $(C, D)$ be an $N$-labelling of $M$. Suppose $\{d, e\}$ is a parallel pair in $M / c$, for some $c \in C$. Let $\left(C^{\prime}, D^{\prime}\right)$ be obtained from $(C, D)$ by switching the $N$-labels on $d$ and $e$; then $\left(C^{\prime}, D^{\prime}\right)$ is an $N$ labelling.

Suppose that $(C, D)$ and $\left(C^{\prime}, D^{\prime}\right)$ are $N$-labellings of $M$, where $\left(C^{\prime}, D^{\prime}\right)$ can be obtained from $(C, D)$ by a sequence of $N$-label switches. We say that $\left(C^{\prime}, D^{\prime}\right)$ is switching-equivalent to $(C, D)$.

Delta-wye exchange. Let $M$ be a matroid with a triangle $T=\{a, b, c\}$. Consider a copy of $M\left(K_{4}\right)$ having $T$ as a triangle with $\left\{a^{\prime}, b^{\prime}, c^{\prime}\right\}$ as the complementary triad labelled such that $\left\{a, b^{\prime}, c^{\prime}\right\},\left\{a^{\prime}, b, c^{\prime}\right\}$ and $\left\{a^{\prime}, b^{\prime}, c\right\}$ are triangles. Let $P_{T}\left(M, M\left(K_{4}\right)\right)$ denote the generalised parallel connection of $M$ with this copy of $M\left(K_{4}\right)$ along the triangle $T$. Let $M^{\prime}$ be the matroid $P_{T}\left(M, M\left(K_{4}\right)\right) \backslash T$ where the elements $a^{\prime}, b^{\prime}$ and $c^{\prime}$ are relabelled as $a, b$ and $c$ respectively. This matroid $M^{\prime}$ is said to be obtained from $M$ by a $\Delta-Y$ exchange on the triangle $T$. Dually, a matroid $M^{\prime \prime}$ is obtained from $M$ by a $Y-\Delta$ exchange on the triad $\{a, b, c\}$ if $\left(M^{\prime \prime}\right)^{*}$ is obtained from $M^{*}$ by a $\Delta-Y$ exchange on $\{a, b, c\}$. 
$N$-grounded triangles and triads. Let $M$ be a 3 -connected matroid and let $N$ be a 3 -connected minor of $M$. We say that a triangle or triad $T$ of $M$ is $N$-grounded if, for all distinct $a, b \in T$, none of $M / a / b, M / a \backslash b, M \backslash a / b$, and $M \backslash a \backslash b$ have an $N$-minor.

Lemma 2.14 ([3, Lemma 3.1]). Let $M$ be a 3-connected matroid with a 3 -connected minor $N$ where $|E(N)| \geq 4$. If $T$ is an $N$-grounded triangle of $M$ with $x \in T$, then $x$ is not $N$-contractible.

We focus on matroids where every triangle or triad is $N$-grounded, due to the following:

Lemma 2.15 ([3, Theorem 3.2]). Let $M$ be a 3-connected matroid, and let $N$ be a 3-connected minor of $M$ with $|E(N)| \geq 4$, where $|E(M)|-|E(N)| \geq 5$. Then either

(i) $M$ has an $N$-detachable pair, or

(ii) there is a matroid $M^{\prime}$ obtained by performing a single $\Delta-Y$ or $Y-\Delta$ exchange on $M$ such that $M^{\prime}$ has an $N$-detachable pair, or

(iii) each triangle or triad of $M$ is $N$-grounded.

\section{Setup}

We work towards a proof of Theorem 1.1 in Section [6. In this section, we lay the ground work.

Lemma 3.1. Let $M$ be a 3-connected matroid, and let $N$ be a 3-connected minor of $M$, where every triangle or triad of $M$ is $N$-grounded, and $|E(N)| \geq 4$. If $|E(M)|-|E(N)| \geq 3$, then, for some $\left(M_{0}, N_{0}\right) \in$ $\left\{(M, N),\left(M^{*}, N^{*}\right)\right\}$, there exist elements $d, d^{\prime} \in E\left(M_{0}\right)$ such that $M_{0} \backslash d$ is 3-connected and $M_{0} \backslash d \backslash d^{\prime}$ has an $N_{0}$-minor.

Proof. Suppose $M$ is a wheel or a whirl. Then every element is in a triangle and a triad. So, by duality, we may assume that $M / x$ has an $N$-minor for some $x \in E(M)$, where $x$ is in a triangle $\{x, y, z\}$. Now $\{y, z\}$ is a parallel pair in $M / x$; thus, as $|E(N)| \geq 4$, the matroid $M / x \backslash y$ has an $N$-minor, up to an $N$-label switch. But then the triangle $\{x, y, z\}$ is not $N$-grounded; a contradiction.

Now, by Seymour's Splitter theorem [9], there exists an element $d$ such that $M_{0} \backslash d$ is 3 -connected and has an $N_{0}$-minor, for some $\left(M_{0}, N_{0}\right) \in$ $\left\{(M, N),\left(M^{*}, N^{*}\right)\right\}$. For notational convenience, we will assume that $\left(M_{0}, N_{0}\right)=(M, N)$. So $M \backslash d$ is 3-connected and has an $N$-minor. If $M \backslash d \backslash d^{\prime}$ has an $N$-minor for any $d^{\prime} \in E(M \backslash d)$, then the lemma holds; so assume otherwise. In particular, as $|E(M)|-|E(N)| \geq 3$, there exist distinct elements $c_{1}, c_{2} \in E(M \backslash d)$ such that $M \backslash d / c_{1} / c_{2}$ has an $N$-minor.

Suppose $M \backslash d$ is a wheel or a whirl. Then $c_{1}$ is in a triangle $\left\{c_{1}, t, t^{\prime}\right\}$ of $M \backslash d$. Since $M \backslash d / c_{1}$ has an $N$-minor and $|E(N)| \geq 4$, the matroid $M \backslash d \backslash t$ has an $N$-minor; a contradiction. So $M \backslash d$ is not a wheel or a whirl. Thus, by Seymour's Splitter Theorem, there exists an element $c \in E(M \backslash d)$ such 
that $M \backslash d / c$ is 3-connected and has an $N$-minor. As $|E(M)|-|E(N)| \geq 3$ and $M \backslash d$ has no $N$-deletable elements, there exists an element $c^{\prime}$ such that $M \backslash d / c / c^{\prime}$ has an $N$-minor. If $M / c$ is 3 -connected, then the lemma holds. So assume that $M / c$ is not 3 -connected. Since $M / c \backslash d$ is 3 -connected, we deduce that $d$ is in a parallel pair in $M / c$, so $\{c, d\}$ is in a triangle of $M$. But $M \backslash d / c$ has an $N$-minor, so this triangle is not $N$-grounded; a contradiction.

Suppose $M$ and $M \backslash d$ are 3-connected matroids, for some $d \in E(M)$, and let $X \subseteq E(M \backslash d)$ be an exactly 3-separating set in $M \backslash d$. We say that $d$ blocks $X$ if $X$ is not 3-separating in $M$. If $d$ blocks $X$, then $d \in \operatorname{cl}^{*}(X)$.

Lemma 3.2. Let $M$ be a 3-connected matroid, and let $N$ be a 3-connected minor of $M$, where every triangle or triad of $M$ is $N$-grounded, and $|E(N)| \geq 4$. Suppose that there exist elements $d, d^{\prime} \in E(M)$ such that $M \backslash d$ is 3-connected and $M \backslash d \backslash d^{\prime}$ has an $N$-minor. Then either:

(i) $M$ has an $N$-detachable pair,

(ii) $M$ has a 4-element cocircuit containing $\left\{d, d^{\prime}\right\}$, or

(iii) $M \backslash d$ has a cyclic 3 -separation $\left(Y,\left\{d^{\prime}\right\}, Z\right)$ such that $|Y \cap E(N)| \leq 1$ and $|Y| \geq 4$.

Proof. If $M \backslash d \backslash d^{\prime}$ is 3-connected, then (i) holds, so assume otherwise. Let $(S, T)$ be a 2-separation of $M \backslash d \backslash d^{\prime}$. Suppose $\operatorname{co}\left(M \backslash d \backslash d^{\prime}\right)$ is 3-connected. Then, without loss of generality, $S$ is contained in a series class in $M \backslash d \backslash d^{\prime}$, and, by Lemma 2.11, $|S \cap E(N)| \leq 1$. Now $M \backslash d \backslash d^{\prime} /(S-s)$ has an $N$-minor for any $s \in S$, since $|E(N)| \geq 4$. Let $S^{\prime}$ be a 2-element subset of $S$. Since $M \backslash d$ is 3-connected, $S^{\prime} \cup d^{\prime}$ is a triad of $M \backslash d$. As $M \backslash d \backslash d^{\prime}$ has an $N$-minor, and every triad of $M$ is $N$-grounded, $d$ blocks $S^{\prime} \cup d^{\prime}$, so $S^{\prime} \cup\left\{d, d^{\prime}\right\}$ is a 4-element cocircuit, in which case (ii) holds.

Now we may assume that $\operatorname{co}\left(M \backslash d \backslash d^{\prime}\right)$ is not 3-connected. Then $M \backslash d$ has a cyclic 3-separation $\left(Y,\left\{d^{\prime}\right\}, Z\right)$ with $|Y \cap E(N)| \leq 1$, by Lemmas 2.6 and 2.11. Suppose that $|Y|=3$. Then $Y$ is a triangle, since $Y$ contains a circuit. But there is at most one element in $Y$ that is not $N$-contractible, by Lemma 2.12, so $Y$ is not an $N$-grounded triangle. Hence $|Y| \geq 4$, and thus (iii) holds.

Lemma 3.3. Let $M$ be a 3-connected matroid, and let $N$ be a 3-connected minor of $M$ such that $|E(N)| \geq 4$, every triangle or triad of $M$ is $N$ grounded, and $|E(M)|-|E(N)| \geq 5$. Then either

(i) $M$ has an $N$-detachable pair;

(ii) up to replacing $(M, N)$ by $\left(M^{*}, N^{*}\right)$, there exists $d \in E(M)$ such that $M \backslash d$ is 3-connected and has a cyclic 3-separation $\left(Y,\left\{d^{\prime}\right\}, Z\right)$ with $|Y| \geq 4$, where $M \backslash d \backslash d^{\prime}$ has an $N$-minor with $|Y \cap E(N)| \leq 1$;

(iii) up to replacing $(M, N)$ by $\left(M^{*}, N^{*}\right), M$ has an augmented quad 3separator $Q \cup z$ such that $z \in \mathrm{cl}^{*}(Q)$ and $M \backslash z$ has an $N$-minor with $|Q \cap E(N)| \leq 1$; or

(iv) there is an $N$-labelling $(C, D)$ of $M$ such that, for every switchingequivalent $N$-labelling $\left(C^{\prime}, D^{\prime}\right)$, 
(a) $M / c$ and $M \backslash d$ are 3 -connected for every $c \in C^{\prime}$ and $d \in D^{\prime}$,

(b) each pair $\left\{c_{1}, c_{2}\right\} \subseteq C^{\prime}$ is contained in a 4-element circuit, and

(c) each pair $\left\{d_{1}, d_{2}\right\} \subseteq D^{\prime}$ is contained in a 4-element cocircuit.

Proof. Assume that neither (i), (ii) nor (iii) holds; we will show that (iv) holds. By Lemma 3.1, we may assume, up to replacing $M$ by $M^{*}$ and $N$ by $N^{*}$, that there is an element $d_{0} \in E(M)$ such that $M \backslash d_{0}$ is 3 -connected and $M \backslash d_{0}$ has at least one $N$-deletable element, $d_{0}^{\prime}$ say. Now, since neither (i) nor (ii) holds, Lemma 3.2 implies that $\left\{d_{0}, d_{0}^{\prime}\right\}$ is contained in a 4-element cocircuit $C_{0}^{*}$. More generally, we have the following:

3.3.1. If $\left\{d, d^{\prime}\right\}$ is a pair of elements such that $M \backslash d$ is 3-connected and $M \backslash d \backslash d^{\prime}$ has an $N$-minor, then $\left\{d, d^{\prime}\right\}$ is contained in a 4-element cocircuit.

We next show the following:

3.3.2. Let $d$ and $d^{\prime}$ be distinct elements of $M$ such that $M \backslash d$ is 3-connected, and $M \backslash d \backslash d^{\prime}$ has an $N$-minor. Then $M \backslash d^{\prime}$ is 3-connected.

Subproof. Suppose $M \backslash d^{\prime}$ is not 3-connected. Since $d^{\prime}$ is $N$-deletable, it is not in a triad, so $\operatorname{co}\left(M \backslash d^{\prime}\right)$ is not 3-connected. Let $\left(Y,\left\{d^{\prime}\right\}, Z\right)$ be a cyclic 3-separation of $M$. We may assume that $|Y \cap E(N)| \leq 1$, by Lemma 2.11. If $Y \cup d^{\prime}$ is not coclosed, then there is some $z \in Z$ such that $\left(Y \cup z,\left\{d^{\prime}\right\}, Z-z\right)$ is a cyclic 3-separation, and $|(Y \cup z) \cap E(N)| \leq 1$ since $|E(N)| \geq 4$. So we may assume that $Y \cup d^{\prime}$ is coclosed. If $Y$ contains a triangle $T$, then $t \in T$ is $N$ contractible in $M \backslash d^{\prime}$, by Lemma 2.12, so $T$ is not an $N$-grounded triangle; a contradiction. So $Y$ does not contain a triangle, which implies that $|Y| \geq 4$. Since $M \backslash d$ is 3-connected, $\left(Y,\left\{d^{\prime}\right\}, Z-d\right)$ or $\left(Y-d,\left\{d^{\prime}\right\}, Z\right)$ is a path of 3-separations in $M \backslash d$, where $d \in Z$ or $d \in Y$ respectively. As $|Z-d| \geq 2$ and $|Y-d| \geq 2$, the element $d^{\prime}$ is a coguts element in either case. Thus, if $r_{M \backslash d}^{*}(Z-d) \geq 3$ and $r_{M \backslash d}^{*}(Y-d) \geq 3$, then this path of 3 -separations is a cyclic 3-separation.

Suppose $d \in Z$. As $d^{\prime} \in \mathrm{cl}_{M \backslash d}^{*}(Z-d)$, it follows that $r_{M \backslash d \backslash d^{\prime}}^{*}(Z-d)=$ $r_{M \backslash d}^{*}(Z-d)-1$. Since $M \backslash d \backslash d^{\prime}$ has an $N$-minor with $|Y \cap E(N)| \leq 1$, and $|E(N)| \geq 4$, we have $r_{M \backslash d \backslash d^{\prime}}^{*}(Z-d) \geq 2$, thus $r_{M \backslash d}^{*}(Z-d) \geq 3$. We deduce that $\left(Y,\left\{d^{\prime}\right\}, Z-d\right)$ is a cyclic 3 -separation. Since $|Y| \geq 4$, (ii) holds; a contradiction.

Now suppose $d \in Y$. If $r_{M \backslash d}^{*}(Y-d) \geq 3$, then $\left(Y-d,\left\{d^{\prime}\right\}, Z\right)$ is a cyclic 3 -separation, and, since $Y-d$ does not contain a triangle, $|Y-d| \geq 4$, so (ii) also holds in this case. Suppose $r_{M \backslash d}^{*}(Y-d)=2$. Now $Y \cup d^{\prime}$ is a corank-3 set in $M$ consisting of at least five elements. Let $Y^{\prime}=\mathrm{cl}^{*}\left(Y \cup d^{\prime}\right)-\left\{d, d^{\prime}\right\}$. Since $r_{M \backslash d \backslash d^{\prime}}^{*}\left(Y^{\prime}\right)=1$, for any $y \in Y^{\prime}$ the matroid $M \backslash d \backslash d^{\prime} /\left(Y^{\prime}-y\right)$ has an $N$-minor. Therefore, any triad contained in $\operatorname{cl}^{*}\left(Y \cup d^{\prime}\right)$ would not be $N$-grounded, so this set contains no triads. In particular, we observe that $Y \cup d^{\prime}$ is a coplane in $M$ consisting of at least five elements.

Suppose $|Y| \geq 5$. Then there exists $P \subseteq Y \cup d^{\prime}$ such that $M^{*} \mid P=$ $U_{3,6}$. Let $X=P-\left\{d, d^{\prime}\right\}$. Recall that $M / p_{1} / p_{2}$ has an $N$-minor for all 
distinct $p_{1}, p_{2} \in X$, and $\mathrm{cl}^{*}(P)$ does not contain any triads. It follows, by Lemma 2.10, that $M$ has an $N$-detachable pair. So we may assume that $|Y|=4$. Since $Y$ does not contain any triangles or triads, $Y$ is a quad in $M$. So $Y \cup d^{\prime}$ is an augmented quad 3-separator, thus (iii) holds; a contradiction.

Let $C_{0}^{*}-\left\{d_{0}, d_{0}^{\prime}\right\}=\left\{c_{0}, e\right\}$. Since $\left\{c_{0}, e\right\}$ is a series pair in $M \backslash d_{0} \backslash d_{0}^{\prime}$, both $M \backslash d_{0} \backslash d_{0}^{\prime} / c_{0}$ and $M \backslash d_{0} \backslash d_{0}^{\prime} / e$ have $N$-minors. So neither $c_{0}$ nor $e$ is contained in a triangle. Both $M \backslash d_{0}$ and $M \backslash d_{0}^{\prime}$ are 3-connected, by 3.3.2, Thus, Lemma 2.9 implies that either $M / c_{0}$ or $M / e$ is 3-connected. Without loss of generality, $M / c_{0}$ is 3 -connected.

Let $(C, D)$ be an $N$-labelling of $M$ with $d_{0} \in D$ and $c_{0} \in C$. For any $d_{1} \in D-d_{0}$, the pair $\left\{d_{0}, d_{1}\right\}$ is contained in a 4 -element cocircuit by 3.3 .1 , and $M \backslash d_{1}$ is 3 -connected by 3.3.2. Now, for any $d_{2} \in D-d_{1}$, the matroid $M \backslash d_{1} \backslash d_{2}$ has an $N$-minor, so $\left\{d_{1}, d_{2}\right\}$ is contained in a 4-element cocircuit, by 3.3.1. Thus every pair of elements in $D$ is contained in a 4-element

cocircuit. A dual argument shows that $M / c$ is 3-connected for every $c \in C$ and every pair $\left\{c_{1}, c_{2}\right\} \subseteq C$ is contained in a 4 -element circuit.

Let $\left(C^{\prime}, D^{\prime}\right)$ be an $N$-labelling of $M$ that is switching-equivalent to $(C, D)$. It remains to show that these properties also hold for $\left(C^{\prime}, D^{\prime}\right)$. It is sufficient to show they hold when $\left(C^{\prime}, D^{\prime}\right)$ is obtained from $(C, D)$ by a single $N$ label switch. Suppose the switch is on an element $d \in D$; that is, $D^{\prime}=$ $(D-d) \cup e$ for some $e \in E(M)-D$. Let $d^{\prime} \in D-d$. Then $M \backslash d^{\prime} \backslash e$ has an $N$-minor, and $M \backslash d^{\prime}$ is 3-connected. By 3.3.2, $M \backslash e$ is 3-connected. So $\left\{d^{\prime}, e\right\}$ is contained in a 4-element cocircuit by 3.3.1, for every $d^{\prime} \in D-d$. The same argument applies for an $N$-label switch on an element in $C$. So (iv) holds, thus completing the proof.

\section{When EVERY $N$-DELETABle PAIR IS IN A 4-ELEMENT COCIRCUIT}

Next, we focus on the case where Lemma 3.3)(iv) holds. In this section, we prove Proposition 4.1. Before stating this proposition, we define some 12element matroids that can have no $N$-detachable pairs, for an appropriately chosen minor $N$.

Definition 4.1. A matroid $M$ is a quad-flower if $|E(M)|=12$ and $r(M)=$ $r^{*}(M)=6$, and there is a partition $\left(Q_{1}, Q_{2}, Q_{3}\right)$ of $E(M)$ such that $Q_{1} \cup Q_{2}$, $Q_{1} \cup Q_{3}$, and $Q_{2} \cup Q_{3}$ are double-quad 3-separators.

We note that a quad-flower $M$ is 3-connected, but neither $M \backslash x \backslash y$ nor $M / x / y$ is 3 -connected, for every pair $\{x, y\} \subseteq E(M)$. Moreover, for a 3connected minor $N$ of $M$, it is possible that $E(N)$ meets each of the three quads that partition $E(M)$.

Definition 4.2. A matroid $M$ is a nest of twisted cubes if $|E(M)|=12$ and $r(M)=r^{*}(M)=6$, and there exists a labelling $\bigcup_{i \in[6]}\left\{e_{i}, e_{i}^{\prime}\right\}$ of $E(M)$ such that $\left\{e_{1}, e_{2}, e_{3}^{\prime}, e_{4}^{\prime}\right\},\left\{e_{3}, e_{4}, e_{5}^{\prime}, e_{6}^{\prime}\right\},\left\{e_{5}, e_{6}, e_{1}^{\prime}, e_{2}^{\prime}\right\}$, $\left\{e_{4}, e_{5}, e_{1}^{\prime}, e_{3}^{\prime}\right\},\left\{e_{1}, e_{3}, e_{6}^{\prime}, e_{2}^{\prime}\right\},\left\{e_{6}, e_{2}, e_{4}^{\prime}, e_{5}^{\prime}\right\}$, 
$\left\{e_{2}, e_{5}, e_{3}^{\prime}, e_{6}^{\prime}\right\},\left\{e_{1}, e_{4}, e_{2}^{\prime}, e_{5}^{\prime}\right\},\left\{e_{3}, e_{6}, e_{1}^{\prime}, e_{4}^{\prime}\right\}$, $\left\{e_{5}, e_{1}, e_{4}^{\prime}, e_{6}^{\prime}\right\},\left\{e_{4}, e_{6}, e_{2}^{\prime}, e_{3}^{\prime}\right\},\left\{e_{2}, e_{3}, e_{5}^{\prime}, e_{1}^{\prime}\right\}$,

$\left\{e_{2}, e_{4}, e_{6}^{\prime}, e_{1}^{\prime}\right\},\left\{e_{6}, e_{1}, e_{3}^{\prime}, e_{5}^{\prime}\right\}$, and $\left\{e_{3}, e_{5}, e_{2}^{\prime}, e_{4}^{\prime}\right\}$ are circuits; and

$\left\{e_{1}, e_{2}, e_{5}^{\prime}, e_{6}^{\prime}\right\},\left\{e_{3}, e_{4}, e_{1}^{\prime}, e_{2}^{\prime}\right\},\left\{e_{5}, e_{6}, e_{3}^{\prime}, e_{4}^{\prime}\right\}$,

$\left\{e_{4}, e_{5}, e_{6}^{\prime}, e_{2}^{\prime}\right\},\left\{e_{1}, e_{3}, e_{4}^{\prime}, e_{5}^{\prime}\right\},\left\{e_{6}, e_{2}, e_{1}^{\prime}, e_{3}^{\prime}\right\}$,

$\left\{e_{2}, e_{5}, e_{1}^{\prime}, e_{4}^{\prime}\right\},\left\{e_{1}, e_{4}, e_{3}^{\prime}, e_{6}^{\prime}\right\},\left\{e_{3}, e_{6}, e_{2}^{\prime}, e_{5}^{\prime}\right\}$,

$\left\{e_{5}, e_{1}, e_{2}^{\prime}, e_{3}^{\prime}\right\},\left\{e_{4}, e_{6}, e_{5}^{\prime}, e_{1}^{\prime}\right\},\left\{e_{2}, e_{3}, e_{4}^{\prime}, e_{6}^{\prime}\right\}$,

$\left\{e_{2}, e_{4}, e_{3}^{\prime}, e_{5}^{\prime}\right\},\left\{e_{6}, e_{1}, e_{2}^{\prime}, e_{4}^{\prime}\right\}$, and $\left\{e_{3}, e_{5}, e_{6}^{\prime}, e_{1}^{\prime}\right\}$ are cocircuits.

A nest of twisted cubes is a 12-element matroid that, for an appropriately chosen 3 -connected minor $N$, has no $N$-detachable pairs. Indeed, let $M$ be a nest of twisted cubes, with labelling as defined above. Then it can be readily checked that $M$ is self-dual under the isomorphism that maps $e_{i}$ to $e_{i}^{\prime}$ and $e_{i}^{\prime}$ to $e_{i}$, for each $i \in[6]$, and $M$ has the property that $M \backslash x \backslash y$ is 3-connected for distinct $x, y \in E(M)$ if and only if $\{x, y\}=\left\{e_{i}, e_{i}^{\prime}\right\}$ for some $i \in[6]$. Moreover, $M$ is 4 -connected. Let $N=U_{2,4}$. Then $M$ has an $N$-minor, but neither $M / e_{i} / e_{i}^{\prime}$ nor $M \backslash e_{i} \backslash e_{i}^{\prime}$ has an $N$-minor for any $i \in[6]$.

Proposition 4.1. Let $M$ be a 3-connected matroid, and let $N$ be a 3connected minor of $M$ such that $|E(N)| \geq 4$, every triangle or triad of $M$ is $N$-grounded, and $|E(M)|-|E(N)| \geq 5$. Let $(C, D)$ be an $N$-labelling of $M$ such that, for every switching-equivalent $N$-labelling $\left(C^{\prime}, D^{\prime}\right)$,

(a) $M / c$ and $M \backslash d$ are 3 -connected for every $c \in C^{\prime}$ and $d \in D^{\prime}$,

(b) each pair $\left\{c_{1}, c_{2}\right\} \subseteq C^{\prime}$ is contained in a 4-element circuit, and

(c) each pair $\left\{d_{1}, d_{2}\right\} \subseteq D^{\prime}$ is contained in a 4-element cocircuit.

Then, up to replacing $(M, N)$ by $\left(M^{*}, N^{*}\right)$, one of the following holds:

(i) there is some $P \subseteq E(M)$ such that $E(M)-E(N) \subseteq P$ and $P$ is a spike-like 3-separator, a double-quad 3-separator, an elongated-quad 3-separator, or a skew-whiff 3-separator;

(ii) there exists $d \in E(M)$ such that $M \backslash d$ is 3-connected and has a cyclic 3-separation $\left(Y,\left\{d^{\prime}\right\}, Z\right)$ with $|Y| \geq 4$, where $M \backslash d \backslash d^{\prime}$ has an $N$ minor with $|Y \cap E(N)| \leq 1$;

(iii) $M$ has an augmented quad 3-separator $Q \cup z$ such that $M$ has an $N$-minor with $|(Q \cup z) \cap E(N)| \leq 1$;

(iv) $|E(M)|=12$, and $M$ is either a quad-flower or a nest of twisted cubes; or

(v) $|E(M)| \leq 10$.

For the entirety of this section we work under the hypotheses of Proposition 4.1. We start with a lemma that provides sufficient conditions for (ii) to hold. We will use this frequently in what follows.

Lemma 4.2. If there exists a pair $\left\{d, d^{\prime}\right\} \subseteq D^{\prime}$, for some $N$-labelling $\left(C^{\prime}, D^{\prime}\right)$ that is switching-equivalent to $(C, D)$, and a set $X \subseteq E(M)-\left\{d, d^{\prime}\right\}$ such that $|X| \geq 4, X$ is 3-separating in $M \backslash d, X$ contains a circuit, $\left|X-\left(C^{\prime} \cup D^{\prime}\right)\right| \leq 2$, and $d^{\prime} \in \mathrm{cl}_{M \backslash d}^{*}(X)$; then (ii) of Proposition 4.1 holds. 
Proof. Let $Y=X \cup\left\{d, d^{\prime}\right\}$ and $Z=E(M)-Y$. We claim that $\left(X,\left\{d^{\prime}\right\}, Z\right)$ is a cyclic 3-separation of $M \backslash d$. Indeed, $X \cup d^{\prime}$ is 3-separating in $M \backslash d$ since $d^{\prime} \in \mathrm{cl}_{M \backslash d}^{*}(X)$ and $M \backslash d$ is 3-connected. So $\left(X,\left\{d^{\prime}\right\}, Z\right)$ is a path of 3-separations in $M \backslash d$ where $d^{\prime}$ is a coguts element. Since $|E(N)| \geq 4$ and $\left|Y-\left(C^{\prime} \cup D^{\prime}\right)\right| \leq 2$, Lemma 2.11 implies that $|X \cap E(N)| \leq 1$. If $r_{M \backslash d}^{*}(Z)=2$, then $r_{M \backslash d \backslash d^{\prime}}^{*}(Z)=1$, so $|Z \cap E(N)| \leq 1$. But this contradicts that $|E(N)| \geq 4$. As $X$ contains a circuit, $\left(X,\left\{d^{\prime}\right\}, Z\right)$ is a cyclic 3 -separation of $M \backslash d$ as claimed, so Proposition 4.1(ii) holds.

Towards a proof of Proposition 4.1, we first handle the case where an $N$-deletable or $N$-contractible pair is contained in a quad.

Lemma 4.3. Suppose $\left(C^{\prime}, D^{\prime}\right)$ is an $N$-labelling of $M$ that is switchingequivalent to $(C, D)$, and there is a pair $\left\{d, d^{\prime}\right\} \subseteq D^{\prime}$ or $\left\{c, c^{\prime}\right\} \subseteq C^{\prime}$ that is contained in a quad. Then, up to replacing $(M, N)$ by $\left(M^{*}, N^{*}\right)$, either

(i) there exists $d_{0} \in E(M)$ such that $M \backslash d_{0}$ is 3-connected and has a cyclic 3-separation $\left(Y,\left\{d_{0}^{\prime}\right\}, Z\right)$ with $|Y| \geq 4$, where $M \backslash d_{0} \backslash d_{0}^{\prime}$ has an $N$-minor with $|Y \cap E(N)| \leq 1$;

(ii) $M$ has an augmented quad 3-separator $Q \cup z$ such that $M$ has an $N$-minor with $|(Q \cup z) \cap E(N)| \leq 1$;

(iii) there is some $P \subseteq E(M)$ such that $E(M)-E(N) \subseteq P$ and $P$ is a spike-like 3-separator, an elongated-quad 3-separator, or a doublequad 3-separator of $M$; or

(iv) $|E(M)| \in\{10,12\}$, and there is a partition $\left(Q_{1}, Q_{2}, Z\right)$ of $E(M)$ such that $Q_{1} \cup Q_{2}$ is a double-quad 3-separator, and $Q_{1} \cup Z$ and $Q_{2} \cup Z$ are both double-quad 3-separators or elongated-quad 3-separators.

Proof. Suppose that (i) does not hold; we will show that (ii), (iii), or (iv) holds. For notational convenience, in what follows we use $(C, D)$, rather than $\left(C^{\prime}, D^{\prime}\right)$, to refer to an $N$-labelling that is switching-equivalent to $(C, D)$.

4.3.1. Let $Q$ be a quad with $|Q \cap(C \cup D)| \geq 2$. If $e \in \operatorname{cl}(Q)-Q$ and $e \in C$, or $e \in \mathrm{cl}^{*}(Q)-Q$ and $e \in D$, then $Q \cup e$ is an augmented quad 3-separator such that (ii) holds.

Subproof. Let $e \in \operatorname{cl}(Q)-Q$ where $e$ is $N$-labelled for contraction. Clearly $Q \cup e$ is an augmented quad 3 -separator. Since $|Q \cap(C \cup D)| \geq 2$ and $|E(N)| \geq$ 4, Lemma 2.11implies that $M / e$ has an $N$-minor such that $|Q \cap E(N)| \leq 1$, so (ii) holds. Similarly, if some $e \in \operatorname{cl}^{*}(Q)-Q$ is $N$-labelled for deletion, then $Q \cup e$ is an augmented quad 3-separator such that (ii) holds.

We may assume, by taking the dual if necessary, that a pair of elements in $D$ is contained in a quad $Q_{1}$. Suppose that $|D|=2$. Let $\left\{d, d^{\prime}\right\} \subseteq D \cap Q_{1}$. Since $|E(M)|-|E(N)| \geq 5$, we have $|C| \geq 3$. Let $c_{1}, c_{2}, c_{3}$ be distinct elements in $C$. For distinct $i, j \in[3]$, the pair $\left\{c_{i}, c_{j}\right\}$ is in a 4-element circuit $C_{i, j}$. Consider the set $X=\left(C_{1,2} \cup C_{1,3} \cup C_{2,3}\right)-\left\{c_{1}, c_{2}, c_{3}\right\}$ in $M / c_{1} / c_{2} / c_{3}$. If $r_{M / c_{1} / c_{2} / c_{3}}(X)=3$, then $X$ consists of three disjoint parallel pairs, so $|D| \geq$ 3 ; a contradiction. So $r_{M / c_{1} / c_{2} / c_{3}}(X) \leq 2$. Then, for some $\{i, j, k\}=[3]$, the 
set $X^{\prime}=\left(C_{i, j} \cup C_{i, k}\right)-\left\{c_{1}, c_{2}, c_{3}\right\}$ satisfies $r_{M / c_{1} / c_{2} / c_{3}}\left(X^{\prime}\right)=1$. If $\left|X^{\prime}\right| \geq 3$, then $\left|X^{\prime} \cap D\right| \geq 2$ and $|D| \geq 3$; a contradiction. So $\left|X^{\prime}\right|=2$; that is, $C_{i, j}=$ $\left\{c_{i}, c_{j}, d_{0}, e\right\}$ and $C_{i, k}=\left\{c_{i}, c_{k}, d_{0}, e\right\}$ for some $d_{0} \in\left\{d, d^{\prime}\right\}$. Now $C_{i, j} \cup C_{i, k}$ is a 5 -element plane that meets $Q_{1}$. By orthogonality, $\left|Q_{1} \cap\left(C_{i, j} \cup C_{i, k}\right)\right| \geq 3$, so $Q_{1} \subseteq \operatorname{cl}_{M}\left(C_{i, j} \cup C_{i, k}\right)$. It now follows from 4.3.1 that $Q_{1} \cup c$ is an augmented quad 3-separator for some $c \in\left\{c_{1}, c_{2}, c_{3}\right\}$, so (ii) holds.

We may now assume that $|D| \geq 3$.

4.3.2. Let $d, d^{\prime}$, and $d^{\prime \prime}$ be distinct elements in $D$, and suppose $\left\{d, d^{\prime}\right\}$ is contained in a quad $Q, d^{\prime \prime} \in E(M)-Q$, and $\left\{d, d^{\prime \prime}\right\}$ is contained in a 4element cocircuit $C^{*}$. Then either $\left|Q \cap C^{*}\right|=2$, or (ii) holds.

Subproof. By orthogonality, $\left|Q \cap C^{*}\right| \neq 1$, so $\left|Q \cap C^{*}\right| \in\{2,3\}$. Suppose $\left|Q \cap C^{*}\right|=3$, in which case $C^{*}-Q=\left\{d^{\prime \prime}\right\}$. Now $d^{\prime \prime} \in \operatorname{cl}^{*}(Q)-Q$ and $d^{\prime \prime}$ is $N$-deletable, so (ii) holds, by 4.3.1.

Recall that there is a pair of elements of $D$ contained in a quad $Q_{1}$. We claim that, up to an $N$-label switch, there are distinct elements $d_{0}, d_{1}, d_{2} \in D$ with $\left\{d_{0}, d_{1}\right\} \subseteq Q_{1}$ and $d_{2} \in E(M)-Q_{1}$. Suppose $\left\{d_{0}, d_{1}\right\}$ is a pair of elements contained in $D \cap Q_{1}$, and $D-Q_{1}=\emptyset$. Since $|D| \geq 3$, there exists an element $d^{\prime} \in D-\left\{d, d_{1}\right\}$, so $d^{\prime} \in Q_{1}$. Let $Q_{1}-\left\{d_{0}, d_{1}, d^{\prime}\right\}=\{c\}$. As $c$ is a coloop in $M \backslash d_{0} \backslash d_{1} \backslash d^{\prime}$, we may assume that $c \in C$. Then, as $|C \cup D| \geq 5$, there exists an element $c^{\prime} \in C-Q_{1}$. Now $\left\{c, c^{\prime}\right\}$ is contained in a 4-element circuit $C_{1}$. By orthogonality and 4.3.1, we may assume that $\left|C_{1} \cap Q_{1}\right|=2$, otherwise (ii) holds. Let $C_{1}-Q_{1}=\left\{c^{\prime}, d_{2}\right\}$. Since $M \backslash d_{0} \backslash d^{\prime} / c / c^{\prime}$ has an $N$-minor, and $d_{2}$ is in a parallel pair in this matroid, we may assume, up to a possible $N$-label switch, that $d_{2} \in D$, and $\left|\left\{d_{0}, d^{\prime}, d_{1}\right\} \cap D\right| \geq 2$. Up to relabelling $d_{0}, d^{\prime}, d_{1}$, we may assume that $d_{0}, d_{1}, d_{2} \in D$ (and $\left\{d_{0}, d_{1}\right\} \subseteq Q_{1}$ ). This proves the claim.

Now let $d_{0}, d_{1}$, and $d_{2}$ be distinct elements of $D$ with $\left\{d_{0}, d_{1}\right\} \subseteq Q_{1}$ and $d_{2} \notin Q_{1}$. In what follows we also assume that (ii) does not hold. If $C_{1}^{*}$ is the 4-element cocircuit containing $\left\{d_{0}, d_{2}\right\}$, then $\left|Q_{1} \cap C_{1}^{*}\right|=2$ by 4.3 .2 , First we rule out the case where $d_{1} \in C_{1}^{*}$. More generally, we prove the following:

4.3.3. Let $d, d^{\prime}, d^{\prime \prime}$ be distinct elements in D. If $\left\{d, d^{\prime}\right\}$ is contained in a quad $Q$, and $\left\{d, d^{\prime \prime}\right\}$ is contained in a 4-element cocircuit $C^{*}$ where $d^{\prime \prime} \notin Q$, then $d^{\prime} \notin C^{*}$.

Subproof. Suppose $d^{\prime} \in C^{*}$. By 4.3.2, $Q \cap C^{*}=\left\{d, d^{\prime}\right\}$. Pick $y$ so that $C^{*}-Q=\left\{y, d^{\prime \prime}\right\}$. Since $M \backslash d \backslash d^{\prime} \backslash d^{\prime \prime}$ has an $N$-minor, and $y$ is a coloop in this matroid, $y$ is $N$-flexible and, in particular, we may assume that $y \in D$, so $C^{*} \subseteq D$. Observe that $Q$ is 3-separating in $M \backslash d^{\prime \prime}$, and $y \in \mathrm{cl}_{M \backslash d^{\prime \prime}}^{*}(Q)$. It follows, by Lemma 4.2, that (i) holds; a contradiction.

We next handle the case where the 4 -element cocircuit containing $\left\{d_{0}, d_{2}\right\}$ is a quad. More generally, we prove the following: 
4.3.4. If there is an element $d^{\prime \prime} \in D-Q_{1}$ such that $\left\{d, d^{\prime \prime}\right\}$ is contained in a quad $Q_{2}$, and $d_{1} \notin Q_{2}$, then $Q_{1} \cup Q_{2}$ is a spike-like 3 -separator where $d_{0}$, $d_{1}$, and $d^{\prime \prime}$ are in different legs.

Subproof. By 4.3.3, $d_{1} \notin Q_{2}$. Observe that $d_{0} \in Q_{1} \cap Q_{2}, d_{1} \in Q_{1}-Q_{2}$ and $d^{\prime \prime} \in Q_{2}-Q_{1}$. By 4.3.2, $\left|Q_{1} \cap Q_{2}\right|=2$. Now $\left\{d_{1}, d^{\prime \prime}\right\}$ is contained in a 4-element cocircuit $C_{1}^{*}$ and, by 4.3 .2 again, $\left|Q_{1} \cap C_{1}^{*}\right|=\left|Q_{2} \cap C_{1}^{*}\right|=2$. By 4.3.3 again, $d_{0} \notin C_{1}^{*}$. Hence, either $C_{1}^{*}=Q_{1} \triangle Q_{2}$, or $C_{1}^{*}=\left\{d_{1}, d^{\prime \prime}, x, q^{\prime}\right\}$ where $\left(Q_{1} \cap Q_{2}\right)-d_{0}=\{x\}$ and $q^{\prime} \in E(M)-\left(Q_{1} \cup Q_{2}\right)$.

We first consider the latter case. Pick $q_{1}$ and $q_{2}$ so that $Q_{1}=\left\{d_{0}, d_{1}, x, q_{1}\right\}$ and $Q_{2}=\left\{d_{0}, x, d^{\prime \prime}, q_{2}\right\}$. As $Q_{1} \cup Q_{2}$ is 3-separating, $q^{\prime} \in \operatorname{cl}^{*}\left(Q_{1} \cup Q_{2}\right)$, and, as $|E(M)| \geq 9$, it follows from Lemma 2.5 that $q^{\prime} \notin \operatorname{cl}\left(Q_{1} \cup Q_{2}\right)$. Since $\left\{q_{1}, q_{2}, q^{\prime}, x\right\}$ is a series class in $M \backslash d_{0} \backslash d_{1} \backslash d^{\prime \prime}$, we have $|E(M)|-|E(N)| \geq 6$, so $|E(M)| \geq 10$. Moreover, we may assume that $\left\{x, q^{\prime}\right\} \subseteq C$, so $\left\{x, q^{\prime}\right\}$ is contained in a 4-element circuit $C_{1}$. By orthogonality, $C_{1}$ meets $Q_{1}-x$ and $Q_{2}-x$. As $q^{\prime} \notin \operatorname{cl}\left(Q_{1} \cup Q_{2}\right)$, we deduce that $C_{1}=\left\{x, q^{\prime}, d_{0}, f\right\}$ where $f \in E(M)-\left(Q_{1} \cup Q_{2} \cup q^{\prime}\right)$.

Since $M \backslash d_{1} \backslash d^{\prime \prime} / x / q^{\prime}$ has an $N$-minor, and $\left\{d_{0}, f\right\}$ is a parallel pair in this matroid, we may assume, up to an $N$-label switch, that $\left\{d_{1}, d^{\prime \prime}, f\right\} \subseteq$ $D$. So $\left\{f, d^{\prime \prime}\right\}$ is contained in a 4 -element cocircuit $C_{2}^{*}$. The cocircuit $C_{2}^{*}$ meets $\left\{x, d_{0}, q_{2}\right\}$, by orthogonality with $Q_{2}$. If $q_{2} \in C_{2}^{*}$, then $C_{2}^{*}$ also meets $\left\{x, d_{0}, q^{\prime}\right\}$, by orthogonality with $C_{1}$. On the other hand, if $q_{2} \notin C_{2}^{*}$, then $C_{2}^{*}$ meets $\left\{x, d_{0}\right\}$, and hence intersects $Q_{1}$ in two elements, by orthogonality. In either case, $C_{2}^{*} \subseteq Q_{1} \cup Q_{2} \cup\left\{q^{\prime}, f\right\}$. Thus $f$ is in the closure and coclosure of the 3-separating set $Q_{1} \cup Q_{2} \cup q^{\prime}$; since $|E(M)| \geq 10$, this is contradictory.

Now we consider the case where $C_{1}^{*}=Q_{1} \triangle Q_{2}$. If $C_{1}^{*}$ is dependent, then $C_{1}^{*}$ is a quad, and $Q_{1} \cup Q_{2}$ is a spike-like 3 -separator, as illustrated in Figure 3. So suppose $C_{1}^{*}$ is independent. We let $Q_{1}-Q_{2}=\left\{q_{1}, d_{1}\right\}$ and $Q_{2}-Q_{1}=\left\{q_{2}, d^{\prime \prime}\right\}$. The matroid $M \backslash d_{0} \backslash d_{1} \backslash d^{\prime \prime} / q_{1} / q_{2}$ has an $N$-minor, so $\left\{q_{1}, q_{2}\right\}$ is contained in a 4 -element circuit $C_{1}$. Since $C_{1}^{*}$ is independent, $C_{1} \neq C_{1}^{*}$, so $C_{1}$ meets $\left\{d_{0}, x\right\}$, by orthogonality. But $\left\{d_{0}, x\right\} \nsubseteq C_{1}$, by 4.3.1. So $C_{1}=\left\{q_{1}, q_{2}, x^{\prime}, f\right\}$, where $x^{\prime} \in\left\{x, d_{0}\right\}$ and $f \in E(M)-\left(Q \cup q_{2}\right)$.

If $x^{\prime} \neq d_{0}$, then it follows that $M \backslash d_{0} \backslash d_{1} \backslash d^{\prime \prime} / x^{\prime} / q_{1}$ has an $N$-minor, where $\left\{q_{2}, f\right\}$ is a parallel pair in this matroid. If $f=d^{\prime \prime}$, then $q_{1} \in \operatorname{cl}\left(Q_{2}\right) \cap C$, in which case (ii) holds by 4.3.1, so we may assume $f \neq d^{\prime \prime}$. Now, up to switching the $N$-labels on $q_{2}$ and $f$, we have $\left\{d^{\prime \prime}, q_{2}\right\} \subseteq D$, in which case (i) holds by Lemma 4.2, a contradiction.

So let $C_{1}=\left\{q_{1}, q_{2}, d_{0}, f\right\}$. Now, as $M \backslash d_{1} \backslash d^{\prime \prime} / q_{1} / q_{2}$ has an $N$-minor, and $\left\{d_{0}, f\right\}$ is a parallel pair in this matroid, $M \backslash d_{1} \backslash d^{\prime \prime} \backslash f / q_{1} / q_{2}$ has an $N$-minor. So $\left\{f, d^{\prime \prime}\right\}$ is contained in a cocircuit $C_{2}^{*}$. Since $Q_{1} \cup Q_{2}$ is 3-separating, and $f \in \operatorname{cl}\left(Q_{1} \cup Q_{2}\right)$, we have $f \notin \operatorname{cl}^{*}\left(Q_{1} \cup Q_{2}\right)$, since $|E(M)| \geq 9$. In particular, $C_{2}^{*} \nsubseteq Q_{1} \cup Q_{2} \cup f$. It follows, by orthogonality between $C_{2}^{*}$ and either $Q_{2}$ or $Q_{1}$, that $q_{2} \in C_{2}^{*}$, and $C_{2}^{*}=\left\{f, q_{2}, d^{\prime \prime}, h\right\}$ for some $h \in$ $E(M)-\left(Q_{1} \cup Q_{2} \cup f\right)$. As $M \backslash d_{1} \backslash d^{\prime \prime} \backslash f / q_{1}$ has an $N$-minor, and $\left\{q_{2}, h\right\}$ is a series pair in this matroid, $M \backslash f / q_{1} / h$ has an $N$-minor. So $\left\{q_{1}, h\right\}$ is 


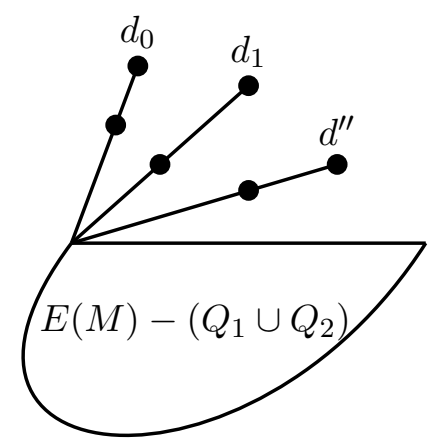

FiguRE 3. The labelling of the spike-like 3-separator that arises in 4.3.4.

contained in a circuit $C_{2}$. By orthogonality with $Q_{1}, Q_{2}$, and $C_{2}^{*}$, we have $C_{2} \subseteq Q_{1} \cup Q_{2} \cup\{f, h\}$, so $h \in \operatorname{cl}\left(Q_{1} \cup Q_{2} \cup f\right) \cap \mathrm{cl}^{*}\left(Q_{1} \cup Q_{2} \cup f\right)$. By Lemma 2.5, we deduce that $|E(M)|=9$. Since $Q_{1} \cup Q_{2}$ and $Q_{1} \cup Q_{2} \cup f$ are exactly 3-separating, $f$ is a guts element, so $E(M)-\left(Q_{1} \cup Q_{2}\right)$ is a triangle containing $\{f, h\}$. But since $M \backslash f / h$ has an $N$-minor, this triangle is not $N$-grounded; a contradiction.

When 4.3.4 holds, it remains to show that $E(M)-E(N)$ is contained in a spike-like 3 -separator.

4.3.5. Suppose $X^{\prime}$ is a spike-like 3-separator where each leg has an element that is $N$-labelled for deletion. Then $X^{\prime}$ is contained in a spike-like 3-separator $X$ such that $E(M)-E(N) \subseteq X$.

Subproof. Let $X^{\prime}$ be a spike-like 3 -separator containing elements $d_{0}, d_{1}$, and $d_{2}$, no two of which is contained in one leg, where $\left\{d_{0}, d_{1}, d_{2}\right\}$ is $N$-labelled for deletion. Let $X$ be a spike-like 3 -separator containing $X^{\prime}$ that is maximal subject to the constraint that each leg has an element that is $N$-labelled for removal. Let $L_{1}, L_{2}, \ldots, L_{t}$ be the legs of the spike-like 3 -separator $X$, where $L_{i}=\left\{d_{i}, c_{i}\right\}$ for each $i \in[t-1]$, and $L_{t}=\left\{d_{0}, x\right\}$. We may assume, up to switching $N$-labels, that $c_{1}$ and $c_{2}$ are $N$-labelled for contraction. Towards a contradiction, suppose there is either some $c^{\prime} \in E(M)-X$ that is $N$-labelled for contraction, or some $d^{\prime} \in E(M)-X$ that is $N$-labelled for deletion.

First, we claim that if there exists some $d^{\prime} \in D-X$, then $d^{\prime}$ is in a 4element cocircuit $C^{*}$ such that $C^{*} \cap X=L_{i}$ for some $i \in[t]$. Let $d^{\prime} \in D-X$. Then $\left\{d^{\prime}, d_{1}\right\},\left\{d^{\prime}, d_{2}\right\}$, and $\left\{d^{\prime}, d_{0}\right\}$ are contained in 4 -element cocircuits. Suppose that each of these cocircuits is contained in $X \cup d^{\prime}$. It follows, by orthogonality with the circuits of $X$, that $t=3$. Since $r^{*}(X)=4$, and $d^{\prime} \in$ $\mathrm{cl}^{*}(X)$, the set $\left\{d^{\prime}, d_{0}, d_{1}, d_{2}, c_{2}\right\}$ contains a cocircuit. But if $\left\{d^{\prime}, d_{1}, d_{2}, c_{2}\right\}$ is a cocircuit, then it intersects the circuit $\left\{x, d_{0}, c_{1}, d_{1}\right\}$ in a single element, contradicting orthogonality. So the cocircuit contained in $\left\{d^{\prime}, d_{0}, d_{1}, d_{2}, c_{2}\right\}$ contains $d_{0}$, and similarly we deduce that it contains $d_{1}$ and $d_{2}$. Thus either $\left\{d^{\prime}, d_{0}, d_{1}, d_{2}\right\},\left\{d_{0}, d_{1}, d_{2}, c_{2}\right\}$, or $\left\{d^{\prime}, d_{0}, d_{1}, d_{2}, c_{2}\right\}$ is a cocircuit. In the first 
case, we may assume that $d_{1}$ is $N$-labelled for contraction, while in the latter two cases, we can swap $N$-labels on $d_{1}$ and $c_{2}$; in any case, $\left\{c_{1}, d_{1}\right\}$ is $N$ labelled for contraction. Observe that $d_{1} \in \mathrm{cl}_{M / c_{1}}\left(X-L_{1}\right)$, so (i) holds by the dual of Lemma 4.2. We deduce that for some $i \in\{0,1,2\}$, the 4-element cocircuit $C_{i}^{*}$ containing $\left\{d^{\prime}, d_{i}\right\}$ is not contained in $X \cup d^{\prime}$. By orthogonality, $C_{i}^{*} \cap X=L_{i}$. This proves the claim.

We can argue similarly in the dual. That is, if there is some $c^{\prime} \in C-X$, then either $c^{\prime}$ is in a 4-element circuit $C$ such that $C \cap X=L_{i}$ for some $i \in[t]$, or $t=3$ and $c^{\prime} \in \operatorname{cl}(X)$. In the latter case, $r\left(X \cup c^{\prime}\right)=4$, so $\left\{c^{\prime}, x, c_{1}, c_{2}, d_{2}\right\}$ contains a circuit. But if $\left\{c^{\prime}, c_{1}, c_{2}, d_{2}\right\}$ is a circuit, then it intersects the cocircuit $\left\{x, d_{0}, c_{1}, d_{1}\right\}$ in a single element, contradicting orthogonality. So the circuit contained in $\left\{c^{\prime}, x, c_{1}, c_{2}, d_{2}\right\}$ contains $x$, and, similarly, it contains $c_{1}$ and $c_{2}$. So either $\left\{c^{\prime}, x, c_{1}, c_{2}\right\},\left\{x, c_{1}, c_{2}, d_{2}\right\}$, or $\left\{c^{\prime}, x, c_{1}, c_{2}, d_{2}\right\}$ is a circuit. In any case, we can perform an $N$-label switch so that $x$ is $N$-labelled for deletion, without affecting the $N$-label on $d_{0}$. Since $\left\{x, d_{0}\right\}$ is $N$-labelled for deletion, (i) holds by Lemma 4.2. So we may assume that if there is some $c^{\prime} \in C-X$, then $c^{\prime}$ is in a 4-element circuit $C$ such that $C \cap X=L_{i}$ for some $i \in[t]$.

Next we claim that if there exists some $d^{\prime} \in D-X$, there also exists some $c^{\prime} \in C-X$. Suppose $d^{\prime} \in D-X$. Without loss of generality, the 4-element cocircuit containing $\left\{d^{\prime}, d_{1}\right\}$ is not contained in $X \cup d^{\prime}$. Let $C^{*}=$ $\left\{d_{1}, c_{1}, d^{\prime}, x^{\prime}\right\}$ be this cocircuit, for some $x^{\prime} \in E(M)-\left(X \cup d^{\prime}\right)$. Now, as $\left\{d_{0}, x, d^{\prime}, x^{\prime}, d_{1}, c_{1}\right\}$ is a corank-4 set where $d_{0}, d^{\prime}$ and $d_{1}$ are $N$-labelled for deletion, we may assume $x^{\prime}$ and $c_{1}$ are $N$-labelled for contraction. As $x^{\prime} \in C-X$, this proves the claim.

Now let $c^{\prime} \in C-X$. Recall that $c^{\prime}$ is in a 4-element circuit $C$ such that $C \cap X=L_{i}$ for some $i \in[t]$. Without loss of generality, $C \cap X=$ $L_{1}$. Let $C=\left\{d_{1}, c_{1}, d^{\prime}, c^{\prime}\right\}$ for some $d^{\prime} \in E(M)-\left(X \cup c^{\prime}\right)$. Pick distinct $i, j \in\{2,3, \ldots, t\}$. By circuit elimination on $C$ and $L_{1} \cup L_{i}$, there is a circuit contained in $L_{i} \cup\left\{c_{1}, c^{\prime}, d^{\prime}\right\}$. But, by orthogonality with the cocircuit $L_{1} \cup L_{j}$, this circuit does not contain $c_{1}$. We deduce that $L_{i} \cup\left\{c^{\prime}, d^{\prime}\right\}$ is a circuit for each $i \in[t]$.

Due to the circuit $L_{1} \cup\left\{c^{\prime}, d^{\prime}\right\}$, we may assume $d^{\prime}$ is $N$-labelled for deletion up to an $N$-label switch with $d_{1}$ (note that $c_{1}$ is an element in $L_{1}$ that is $N$-labelled for contraction). Thus $\left\{d^{\prime}, d_{0}\right\}$ is contained in a 4 -element cocircuit $C^{*}$ such that $C^{*} \cap X=L_{t}$. Moreover, by orthogonality with the circuit $\left\{c_{2}, d_{2}, c^{\prime}, d^{\prime}\right\}$, the cocircuit $C^{*}$ meets $\left\{c_{2}, d_{2}, c^{\prime}\right\}$, so $C^{*}=\left\{d_{0}, x, d^{\prime}, c^{\prime}\right\}$. By cocircuit elimination with the cocircuits in $X$, and orthogonality with the circuits in $X$, it follows that $L_{i} \cup\left\{d^{\prime}, c^{\prime}\right\}$ is a cocircuit for each $i \in[t]$. Now $X \cup\left\{c^{\prime}, d^{\prime}\right\}$ is a spike-like 3 -separator, where $c^{\prime}$ is $N$-labelled for contraction, so $X$ is not maximal; a contradiction.

Now, by 4.3.4 and 4.3.5. if the 4-element cocircuit $C_{1}^{*}$ containing $\left\{d_{0}, d_{2}\right\}$ is a quad, then (iii) holds. Next we handle the case where $C_{1}^{*}$ is independent. 
We break it into two parts: first, the case where $Q_{1} \cup C_{1}^{*}$ is not 3-separating; and second, the case where $\lambda\left(Q_{1} \cup C_{1}^{*}\right)=2$.

4.3.6. If $\left\{d_{0}, d_{2}\right\}$ is contained in a 4-element independent cocircuit $C_{1}^{*}$, where $d_{1} \notin C_{1}^{*}$, then either

(I) $\lambda\left(Q_{1} \cup C_{1}^{*}\right)=2$, or

(II) $Q_{1} \cup C_{1}^{*}$ is contained in a double-quad 3-separator $Y$, and either $E(M)-E(N) \subseteq Y$, or $|E(M)| \in\{10,12\}$ and (iv) holds.

Subproof. Suppose that (I) does not hold; we will show that (II) holds. Recall that $\left\{d_{0}, d_{1}\right\}$ is contained in the quad $Q_{1}$, and $d_{2} \notin Q_{1}$. Let $X=$ $Q_{1} \cup C_{1}^{*}$, and pick $c_{1}$ and $c_{2}$ so that $X-C_{1}^{*}=\left\{c_{1}, d_{1}\right\}$ and $X-Q_{1}=\left\{c_{2}, d_{2}\right\}$. If $r(X)=4$, then $\lambda(X)=2$; so we may assume that $r(X)=5$. Since $M \backslash d_{0} \backslash d_{1} \backslash d_{2}$ has an $N$-minor, and $X-\left\{d_{0}, d_{1}, d_{2}\right\}$ has corank one in this matroid, $M \backslash d_{0} \backslash d_{1} \backslash d_{2} / c_{1} / c_{2}$ has an $N$-minor. Now $\left\{c_{1}, c_{2}\right\}$ is contained in a 4-element circuit $C_{1}$. If $C_{1} \subseteq X$, then $r(X)=4$; a contradiction. Thus, by orthogonality, $\left|Q_{1} \cap C_{1}^{*} \cap C_{1}\right|=1$.

Let $C_{1}=\left\{c_{1}, c_{2}, x^{\prime}, e\right\}$, where $x^{\prime} \in Q_{1} \cap C_{1}^{*}$ and $e \in E(M)-X$. Since $M \backslash d_{1} \backslash d_{2} / c_{1} / c_{2}$ has an $N$-minor, and $\left\{e, x^{\prime}\right\}$ is a parallel pair in this matroid, $M \backslash d_{1} \backslash d_{2} \backslash e / c_{1} / c_{2}$ has an $N$-minor. So $\left\{e, d_{1}\right\}$ is contained in a 4 -element cocircuit $C_{2}^{*}$. If $C_{2}^{*} \subseteq Q_{1} \cup C_{1}$, then $Q_{1} \cup C_{1}$ has rank and corank at most four, so $Q_{1} \cup C_{1}$ is 3-separating. Similarly, if $C_{2}^{*} \subseteq Q_{1} \cup C_{1} \cup d_{2}$, then $Q_{1} \cup C_{1} \cup d_{2}$ is 3-separating in $M$, and $d_{2} \notin \operatorname{cl}\left(Q_{1} \cup C_{1}\right)$, so $Q_{1} \cup C_{1}$ is also 3-separating. Since $M \backslash d_{1}$ is 3-connected, it follows that $\lambda_{M \backslash d_{1}}\left(\left(Q_{1}-d_{1}\right) \cup C_{1}\right)=2$, and $d_{2} \in \mathrm{cl}_{M \backslash d_{1}}^{*}\left(\left(Q_{1}-d_{1}\right) \cup C_{1}\right)$, in which case (i) holds by Lemma 4.2. Thus we may assume that $C_{2}^{*} \nsubseteq Q_{1} \cup C_{1} \cup d_{2}$. By orthogonality, we deduce that $C_{2}^{*}$ meets $\left\{x^{\prime}, c_{1}\right\}$, and $f \in C_{2}^{*}$ for some $f \in E(M)-\left(Q_{1} \cup C_{1} \cup d_{2}\right)$.

Let $Y=Q_{1} \cup C_{1} \cup\left\{f, d_{2}\right\}$. Since $M \backslash d_{1} \backslash e / c_{2}$ has an $N$-minor, and $f$ is in a series pair in this matroid, $M / c_{2} / f$ has an $N$-minor, so $\left\{c_{2}, f\right\}$ is contained in a 4-element circuit $P$. By orthogonality, $P$ meets $C_{1}^{*}-c_{2}$ and $C_{2}^{*}-f$. Since $x^{\prime}$ is the only possible element in $\left(C_{1}^{*}-c_{2}\right) \cap\left(C_{2}^{*}-f\right)$, we see that $P \subseteq Y$ when $x^{\prime} \notin P$. But if $x^{\prime} \in P$, then $P$ also meets $Q_{1}-x^{\prime}$ by orthogonality, in which case $P \subseteq Y$. So $r(Y)=5$, and it follows that $r^{*}(Y)=5$ and $\lambda(Y)=2$. In the case that $P$ meets $Q_{1}$, we have $r\left(Y-d_{2}\right)=4$, so $d_{2} \notin \operatorname{cl}\left(Y-d_{2}\right)$. It follows that $Y-\left\{d_{1}, d_{2}\right\}$ is 3 -separating in $M \backslash d_{1}$, and $d_{2} \in \mathrm{cl}_{M \backslash d_{1}}^{*}\left(Y-\left\{d_{1}, d_{2}\right\}\right)$. By another application of Lemma 4.2, (i) holds.

In the remaining case, $P=\left\{c_{2}, e, f, d_{2}\right\}$. Recall that $x^{\prime} \in Q_{1} \cap C_{1}$, and choose $x$ so that $Q_{1} \cap C_{1}=\left\{x, x^{\prime}\right\}$. If $d_{0}=x$, then $M \backslash d_{0} \backslash d_{1} \backslash d_{2} / c_{1} / x^{\prime}$ has an $N$-minor and $\left\{c_{2}, e\right\}$ is a parallel pair in this matroid, so $M \backslash d_{2} \backslash c_{2}$ has an $N$-minor. Then $Q_{1}$ is 3-separating in $M \backslash d_{2}$ with $c_{2} \in \mathrm{cl}_{M \backslash d_{2}}^{*}\left(Q_{1}\right)$, and it follows that (i) holds by Lemma 4.2. So we may assume that $d_{0}=x^{\prime}$. Recall also that $C_{2}^{*}$ meets $\left\{d_{0}, c_{1}\right\}$. In a similar vein, if $d_{0} \in C_{2}^{*}$, then $\{e, f\}$ is a series pair in $M \backslash d_{0} \backslash d_{1} \backslash d_{2} / c_{1}$, so $M \backslash d_{2} / e / c_{1}$ has an $N$-minor, and hence $M \backslash d_{2} \backslash c_{2}$ has an $N$-minor. As before, in this case (i) holds by Lemma 4.2 . So we may assume that $c_{1} \in C_{2}^{*}$. 
We work towards showing that $Y$ is a double-quad 3-separator of $M$, labelled as illustrated in Figure 4 . The pair $\left\{d_{1}, d_{2}\right\}$ is in a 4-element cocircuit $C_{3}^{*}$ that meets both $Q_{1}-d_{1}$ and $P-d_{2}$, by orthogonality. As these sets are disjoint, $C_{3}^{*} \subseteq Y$. If $c_{2} \notin C_{3}^{*}$, then $d_{2} \in \mathrm{cl}^{*}\left(Q_{1} \cup C_{2}^{*}\right)$, and it follows that $r^{*}(Y) \leq 4$; a contradiction. So $c_{2} \in C_{3}^{*}$. Now, if $c_{1} \notin C_{3}^{*}$, then $d_{1} \in \operatorname{cl}^{*}\left(C_{1}^{*}\right)$, and it follows that $r^{*}(Y) \leq 4$; a contradiction. Note also that $x \notin C_{3}^{*}$, for otherwise $r^{*}(X)=3$ and $\lambda(X)=2$. So $C_{3}^{*}=\left\{d_{1}, d_{2}, c_{1}, c_{2}\right\}$.

Recall that $\left\{c_{2}, x\right\}$ is contained in a series class of size at least three in $M \backslash d_{0} \backslash d_{1} \backslash d_{2}$, so $\left\{c_{2}, x\right\}$ is $N$-contractible, and hence is contained in a 4-element circuit $C_{2}$. By orthogonality with $Q_{1}$ and $C_{3}^{*}$, either $C_{2}$ meets $\left\{c_{1}, d_{1}\right\}$, or $C_{2}=C_{1}^{*}$. But $C_{1}^{*}$ is independent, so the former case holds. By orthogonality with $C_{2}^{*}$, we see that $C_{2} \subseteq C_{2}^{*} \cup\left\{c_{2}, x\right\}$. Moreover, $C_{2}$ meets $\{e, f\}$, otherwise $C_{2} \subseteq Q_{1} \cup c_{2}$, which contradicts 4.3.1. If $f \in C_{2}$, then $f \in \operatorname{cl}\left(Q_{1} \cup C_{1}\right)$, and it follows that $r(Y) \leq 4$; a contradiction. So $e \in C_{2}$. Now, if $c_{1} \in C_{2}$, then $x \in \operatorname{cl}\left(C_{1}\right)$, and it follows that $r\left(Q_{1} \cup C_{1}\right) \leq 3$; a contradiction. So $C_{2}=\left\{c_{2}, x, e, d_{1}\right\}$.

Now, as $M \backslash d_{0} / c_{2} / x$ has an $N$-minor, and $\left\{e, d_{1}\right\}$ is a parallel pair in this matroid, $M \backslash d_{0} \backslash e$ has an $N$-minor. So $\left\{d_{0}, e\right\}$ is contained in a 4-element cocircuit $C_{4}^{*}$. By orthogonality, this cocircuit meets $C_{2}-e=\left\{c_{2}, x, d_{1}\right\}$ and $Q_{1}-d_{0}=\left\{c_{1}, x, d_{1}\right\}$, so either $C_{4}^{*}=\left\{d_{0}, e, c_{1}, c_{2}\right\}$, or $C_{4}^{*}$ meets $\left\{x, d_{1}\right\}$. In the former case, $e \in \mathrm{cl}^{*}\left(Q_{1} \cup C_{1}^{*}\right)$, and it follows that $r^{*}(Y) \leq 4$; a contradiction. So $C_{4}^{*}$ meets $\left\{x, d_{1}\right\}$. By orthogonality with $P$, the cocircuit $C_{4}^{*}$ also meets $\left\{c_{2}, f, d_{2}\right\}$. If $f \notin C_{4}^{*}$, then $e \in \mathrm{cl}^{*}\left(Q_{1} \cup C_{1}^{*}\right)$, and it follows that $r^{*}(Y) \leq 4$; a contradiction. So $f \in C_{4}^{*}$. Now, if $d_{1} \in C_{4}^{*}$, then $r^{*}\left(C_{2}^{*} \cup Q_{1}\right)=$ 3 , so $r^{*}(Y) \leq 4$; a contradiction. We deduce that $C_{4}^{*}=\left\{d_{0}, e, f, x\right\}$.

Recall that $M \backslash d_{1} \backslash d_{2} \backslash e$ has an $N$-minor, and note that $\left\{c_{2}, f, c_{1}\right\}$ is contained in a series class in this matroid, since $r^{*}\left(C_{2}^{*} \cup C_{3}^{*}\right)=4$. Thus $M / c_{1} / f$ has an $N$-minor, so $\left\{c_{1}, f\right\}$ is contained in a 4-element circuit $C_{3}$. By orthogonality, $C_{3}$ meets $Q_{1}-c_{1}$ and $C_{4}^{*}-f$. Thus, either $C_{3}=\left\{c_{1}, f, d_{1}, e\right\}$, or $C_{3}$ meets $\left\{d_{0}, x\right\}$. In the former case, $f \in \operatorname{cl}\left(Q_{1} \cup C_{1}\right)$, and it follows that $r(Y) \leq 4$; a contradiction. So $C_{3}$ meets $\left\{d_{0}, x\right\}$, and hence, by orthogonality with $C_{1}^{*}$, the circuit $C_{3}$ also meets $\left\{c_{2}, d_{2}\right\}$. If $c_{2} \in C_{3}$, then $f \in \operatorname{cl}\left(Q_{1} \cup C_{1}\right)$, and it follows that $r(Y) \leq 4$; a contradiction. So $d_{2} \in C_{3}$. Now, if $x \in C_{3}$, then $x \in \operatorname{cl}\left(P \cup C_{1}\right)$, and $r(Y) \leq 4$; a contradiction. So $C_{3}=\left\{c_{1}, f, d_{0}, d_{2}\right\}$.

Since $M \backslash d_{1} \backslash e / c_{1} / f$ has an $N$-minor, and $\left\{d_{0}, d_{2}\right\}$ is a parallel pair in this matroid, $M \backslash d_{0} \backslash d_{1} \backslash e$ has an $N$-minor. As $r^{*}\left(Q_{1} \cup C_{4}^{*}\right)=4$, we see that $\left\{c_{1}, x, f\right\}$ is contained in a series class in $M \backslash d_{0} \backslash d_{1} \backslash e$, so $\{x, f\}$ is $N$ contractible. Now $\{x, f\}$ is contained in a 4-element circuit $C_{4}$. By orthogonality with the cocircuits $Q_{1}$ and $C_{2}^{*}$, either $C_{4}=\left\{x, f, d_{0}, e\right\}$, or $C_{4}$ meets $\left\{c_{1}, d_{1}\right\}$. In the former case, $C_{4}=C_{4}^{*}$ is a quad, and it follows that (iii) holds by 4.3.4 and 4.3.5. In the latter case, $C_{4}$ meets $\left\{c_{2}, d_{2}\right\}$, by orthogonality with the cocircuits $C_{1}^{*}$ and $C_{3}^{*}$. If $c_{1} \in C_{4}$, then $x \in \operatorname{cl}\left(P \cup C_{3}\right)$, and it follows that $r(Y) \leq 4$; a contradiction. So $d_{1} \in C_{4}$. Likewise, if $c_{2} \in C_{4}$, then $f \in \operatorname{cl}\left(Q_{1} \cup C_{1}\right)$, and $r(Y) \leq 4$; a contradiction. So $C_{4}=\left\{x, f, d_{1}, d_{2}\right\}$. 
Finally, we show that $P$ is a cocircuit. As $\left\{e, d_{2}\right\}$ is $N$-deletable, there is certainly a 4 -element cocircuit $C_{5}^{*}$ containing $\left\{e, d_{2}\right\}$. By orthogonality with the circuits $C_{1}$ and $C_{3}$, either $P=C_{5}^{*}$, or $C_{5}^{*}$ meets $\left\{c_{1}, d_{0}\right\}$. Similarly, due to the circuits $C_{2}$ and $C_{4}$, either $P=C_{5}^{*}$, or $C_{5}^{*}$ meets $\left\{d_{1}, x\right\}$. So, if $P \neq C_{5}^{*}$, then $C_{5}^{*} \subseteq Q_{1} \cup\left\{e, d_{2}\right\}$, in which case $e \in \mathrm{cl}^{*}\left(Q_{1} \cup C_{1}^{*}\right)$, implying $r^{*}(Y) \leq 4$; a contradiction. We deduce that $P$ is a cocircuit, and hence $Y$ is a double-quad 3 -separator of $M$.

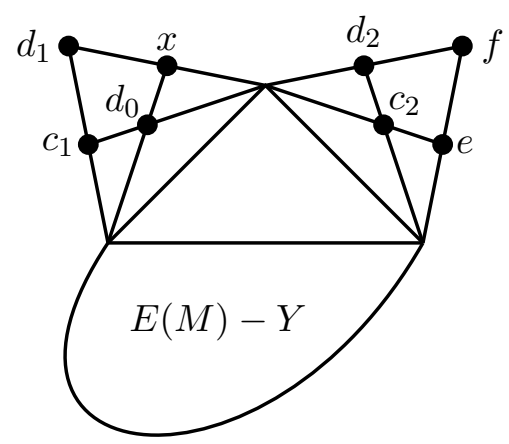

Figure 4. The labelling of the double-quad 3-separator in 4.3.6, where $\left\{d_{0}, d_{1}, d_{2}\right\} \subseteq D$ and $\left\{c_{1}, c_{2}\right\} \subseteq C$.

Suppose that $E(M)-E(N) \nsubseteq Y$. It remains to show that $|E(M)| \in$ $\{10,12\}$ and (iv) holds. Let $(C, D)$ be an $N$-labelling of $M$ with $\left\{c_{1}, c_{2}\right\} \subseteq C$ and $\left\{d_{0}, d_{1}, d_{2}\right\} \subseteq D$, and observe that there exists either some $c^{\prime} \in C-Y$ or some $d^{\prime} \in D-Y$.

First, we show that if there is some such $c^{\prime}$, then any 4-element circuit containing $c^{\prime}$ and an element of $C \cap Y$ is not contained in $Y \cup c^{\prime}$. Consider the 4-element circuit $C_{5}$ containing $\left\{c^{\prime}, c_{1}\right\}$. If this circuit does not contain $d_{1}$, then, by orthogonality, it meets $\left\{d_{0}, x\right\},\left\{d_{2}, c_{2}\right\}$, and $\{e, f\}$; a contradiction. So $d_{1} \in C_{5}$. Now $C_{5}$ meets $C_{1}^{*}$ and $P$ in at most one element, so $C_{5} \cap$ $\left(C_{1}^{*} \cup P\right)=\emptyset$, and thus $C_{5} \cap Y=\left\{c_{1}, d_{1}\right\}$. The argument is similar when considering the 4-element circuit containing $\left\{c^{\prime}, c\right\}$ for some $c \in C \cap\left(Y-c_{1}\right)$, where in each case the circuit intersects $Y$ in one of $\left\{c_{1}, d_{1}\right\},\left\{d_{0}, x\right\},\left\{c_{2}, d_{2}\right\}$, or $\{e, f\}$. A dual argument shows that if there exists some $d^{\prime} \in D-Y$, then any 4-element cocircuit containing $d^{\prime}$ and an element in $D \cap Y$ intersects $Y$ in one of $\left\{d_{1}, x\right\},\left\{c_{1}, d_{0}\right\},\left\{d_{2}, f\right\}$, or $\left\{c_{2}, e\right\}$.

Suppose there exists some $d^{\prime} \in D-Y$. Then $d^{\prime}$ is contained in a 4element cocircuit with $d_{1}$, and hence $x$. Let this cocircuit be $\left\{d^{\prime}, d_{1}, x, c^{\prime}\right\}$ for $c^{\prime} \in E(M)-Y$. Up to an $N$-label switch on $x$ and $c^{\prime}$, the element $c^{\prime}$ is $N$-labelled for contraction. So we may assume that there is some $c^{\prime} \in C-Y$.

Let $C_{5}$ be the 4-element circuit containing $\left\{c^{\prime}, c_{1}\right\}$. Recall that $C_{5}=$ $\left\{c^{\prime}, z_{1}, c_{1}, d_{1}\right\}$ for some $z_{1} \in E(M)-\left(Y \cup c^{\prime}\right)$. Let $C_{6}$ be the 4-element circuit containing $\left\{c^{\prime}, c_{2}\right\}$; then $C_{6}=\left\{c^{\prime}, z_{2}, c_{2}, d_{2}\right\}$ for some $z_{2} \in E(M)-$ $\left(Y \cup c^{\prime}\right)$. By circuit elimination and orthogonality, $C_{7}=\left\{c^{\prime}, z_{1}, d_{0}, x\right\}$ and $C_{8}=\left\{c^{\prime}, z_{2}, e, f\right\}$ are also circuits. Now $r\left(Y \cup\left\{c^{\prime}, z_{1}, z_{2}\right\}\right) \leq r(Y)+1=6$. 
Due to the circuit $C_{5}$, we may assume $\left\{z_{1}, d_{2}\right\}$ is $N$-labelled for deletion, up to an $N$-label switch. Consider the 4-element cocircuit $C_{6}^{*}$ containing $\left\{z_{1}, d_{2}\right\}$. This cocircuit contains $f$. By orthogonality with $C_{5}$, the cocircuit $C_{6}^{*}$ meets $\left\{c^{\prime}, c_{1}, d_{1}\right\}$, and by orthogonality with $C_{6}$, it meets $\left\{c^{\prime}, c_{2}, z_{2}\right\}$. So $C_{6}^{*}=\left\{z_{1}, c^{\prime}, d_{2}, f\right\}$. Similarly, due to the circuit $C_{6}$, we may assume $\left\{z_{2}, d_{1}\right\}$ is $N$-labelled for deletion. Now consider the 4-element cocircuit $C_{7}^{*}$ containing $\left\{z_{2}, d_{1}\right\}$. In a similar fashion, we deduce that $x \in C_{7}^{*}$ and, by orthogonality with $C_{5}$ and $C_{6}$, the final element of $C_{7}^{*}$ is $c^{\prime}$. So $C_{7}^{*}=\left\{z_{2}, c^{\prime}, d_{1}, x\right\}$. By cocircuit elimination and orthogonality, $C_{8}^{*}=\left\{z_{1}, c^{\prime}, c_{2}, e\right\}$ and $C_{9}^{*}=\left\{z_{2}, c^{\prime}, c_{1}, d_{0}\right\}$ are also cocircuits.

Now $z_{1} \in \mathrm{cl}^{*}\left(Y \cup c^{\prime}\right)$, implying $r^{*}\left(Y \cup\left\{c^{\prime}, z_{1}\right\}\right) \leq r^{*}(Y)+1=6$. If $z_{1}=z_{2}$, then $\left\{c^{\prime}, z_{1}, c_{1}, d_{0}, c_{2}\right\}$ spans $Y \cup\left\{c^{\prime}, z_{1}\right\}$, so $r\left(Y \cup\left\{c^{\prime}, z_{1}\right\}\right)=5$, implying $\lambda\left(Y \cup\left\{c^{\prime}, z_{1}\right\}\right) \leq 5+6-10=1$. Hence $|E(M)| \in\{10,11\}$. If $|E(M)|=10$, then $Q \cup\left\{c^{\prime}, z_{1}\right\}$ and $P \cup\left\{c^{\prime}, z_{1}\right\}$ are elongated-quad 3-separators, so (iv) holds.

Suppose that $|E(M)|=11$. Let $E(M)-\left(Y \cup\left\{c^{\prime}, z_{1}\right\}\right)=\{q\}$. If $q \notin \operatorname{cl}(Q)$, then $r(Q \cup q)=4=r(M)-1$, $\operatorname{socl}(Q \cup q)$ is a hyperplane. But $E(M)-(Q \cup q)$ is the union of cocircuits $P$ and $C_{6}^{*}$; a contradiction. So $q$ is in a circuit properly contained in $Q \cup q$. By orthogonality with $C_{1}^{*}$ and $C_{2}^{*}$, it follows that $q$ is in a triangle with $\left\{c_{1}, d_{1}\right\}$ or $\left\{d_{0}, x\right\}$. But $c_{1}$ and $x$ are $N$-contractible, so this triangle is not $N$-grounded; a contradiction.

We may now assume that $z_{1} \neq z_{2}$. So $r^{*}\left(Y \cup\left\{c^{\prime}, z_{1}, z_{2}\right\}\right) \leq r^{*}(Y)+1=6$. Now $\lambda\left(Y \cup\left\{c^{\prime}, z_{1}, z_{2}\right\}\right) \leq 6+6-11=1$, so $|E(M)| \in\{11,12\}$. If $|E(M)|=11$, then $\left\{c^{\prime}, z_{1}, z_{2}\right\}$ is a triad, but $z_{1}$ is $N$-deletable so this triad is not $N$-grounded; a contradiction. So $|E(M)|=12$, and it follows that $Z=$ $E(M)-Y$ is a quad. Pick $q$ so that $Z=\left\{q, c^{\prime}, z_{1}, z_{2}\right\}$. By (co)circuit elimination and orthogonality, it is easily checked that $\left\{q, z_{2}, c_{1}, d_{1}\right\},\left\{q, z_{2}, d_{0}, x\right\}$, $\left\{q, z_{1}, c_{2}, d_{2}\right\}$, and $\left\{q, z_{1}, e, f\right\}$ are circuits, and $\left\{q, z_{2}, d_{2}, f\right\},\left\{q, z_{2}, c_{2}, e\right\}$, $\left\{q, z_{1}, c_{1}, d_{0}\right\}$, and $\left\{q, z_{1}, d_{1}, x\right\}$ are cocircuits, so $Q \cup Z$ and $P \cup Z$ are doublequad 3-separators, and (iv) holds.

4.3.7. Suppose $\left\{d_{0}, d_{2}\right\}$ is contained in a 4-element independent cocircuit $C_{1}^{*}$, where $d_{1} \notin C_{1}^{*}$. Let $X=Q_{1} \cup C_{1}^{*}$. If $\lambda(X)=2$, then $X$ is an elongated-quad 3-separator where either $E(M)-E(N) \subseteq X$, or $|E(M)|=10$ and (iv) holds.

Subproof. Pick $c_{1}, c_{2}$ and $x$ so that $X-C_{1}^{*}=\left\{c_{1}, d_{1}\right\}, X-Q_{1}=\left\{c_{2}, d_{2}\right\}$, and $Q_{1} \cap C_{1}^{*}=\left\{d_{0}, x\right\}$. By 4.3.1 and 4.3.2, $r(X) \geq 4$ and $r^{*}(X) \geq 4$. Since $\lambda(X)=2$, we have $r(X)=r^{*}(X)=4$. As $M \backslash d_{0} \backslash d_{1} \backslash d_{2}$ has an $N$-minor, any pair of elements contained in $\mathrm{cl}^{*}(X)-\left\{d_{0}, d_{1}, x\right\}$ is $N$-contractible in this matroid, so such a pair is contained in a 4-element circuit. In particular, there are 4-element circuits $C_{1}$ and $C_{2}$ containing $\left\{c_{1}, c_{2}\right\}$ and $\left\{c_{2}, x\right\}$, respectively. By orthogonality with the cocircuit $Q_{1}$, these two circuits intersect $X$ in at least three elements. We will show that we may assume that $C_{i} \subseteq X$, for $i \in\{1,2\}$. 
First, we claim that $Q_{1} \triangle C_{1}^{*}$ is a cocircuit. As the pair $\left\{d_{1}, d_{2}\right\}$ is $N$ deletable, it is contained in a 4 -element cocircuit $C_{2}^{*}$. Suppose $C_{2}^{*} \nsubseteq X$. By orthogonality, $\left|C_{2}^{*}-X\right|=1$. Let $C_{2}^{*}-X=\{q\}$. Since $q \in \mathrm{cl}^{*}(X)$, the set $X \cup$ $q$ is 3-separating, and the pair $\left\{c_{2}, q\right\}$ is contained in a 4-element circuit $P$. If $P \subseteq X \cup q$, then $q \in \operatorname{cl}(X) \cap \operatorname{cl}^{*}(X)$, so $\lambda(X \cup q)=1$ and $|E(M)| \leq 8$; a contradiction. It follows, by orthogonality, that $P=\left\{c_{2}, d_{2}, q, f\right\}$, for some $f \in E(M)-(X \cup q)$. Recall that $M \backslash d_{0} \backslash d_{1} / c_{2} / q$ has an $N$-minor. By swapping the $N$-labels on $d_{2}$ and $f$, we deduce that $\left\{d_{1}, f\right\}$ is $N$-deletable, so this pair is contained in a 4-element cocircuit $C_{3}^{*}$. By orthogonality with the disjoint circuits $Q_{1}$ and $P$, the cocircuit $C_{3}^{*}$ is contained in $X \cup\{q, f\}$. Now $f \in \operatorname{cl}(X \cup q) \cap \mathrm{cl}^{*}(X \cup q)$, so, by Lemma 2.5, $|E(M)|=9$. Then, since $X$ is exactly 3 -separating and $q$ is a coguts element, $E(M)-X$ is a triad containing $f$, but $M \backslash f$ has an $N$-minor, so this triad is not $N$-grounded. From this contradiction we deduce that $C_{2}^{*} \subseteq X$. Since $r^{*}(X)=4$, it follows that $C_{2}^{*}=Q_{1} \triangle C_{1}^{*}$, as claimed.

Suppose $\left\{c_{1}, c_{2}, x, e\right\}$ is a circuit, for some $e \in E(M)-X$. Since $M \backslash d_{0} \backslash d_{1} \backslash d_{2} / x / c_{1}$ has an $N$-minor, and $\left\{c_{2}, e\right\}$ is a parallel pair in this matroid, $M \backslash d_{2} \backslash c_{2}$ has an $N$-minor. As $Q_{1}$ is 3 -separating in $M \backslash d_{2}$ with $c_{2} \in \mathrm{cl}_{M \backslash d_{2}}^{*}\left(Q_{1}\right)$, it follows that (i) holds by Lemma 4.2.

Now, if $C_{i} \nsubseteq X$, for some $i \in\{1,2\}$, then either $C_{1}=\left\{c_{1}, c_{2}, d_{0}, e\right\}$, or $C_{2}=\left\{c_{2}, x, d_{1}, e\right\}$, for some $e \in E(M)-X$. In either case, we have that $M \backslash d_{2} \backslash e / c$ has an $N$-minor, for some $c \in\left\{c_{1}, x\right\}$. Indeed, in the first case, $M \backslash d_{2} \backslash e / c_{1}$ has an $N$-minor by swapping the $N$-labels on $d_{0}$ and $e$; in the second, $M \backslash d_{2} \backslash e / x$ has an $N$-minor, by switching the $N$-labels on $d_{1}$ and $e$. So, in either case, $\left\{e, d_{2}\right\}$ is contained in a 4-element cocircuit $C_{3}^{*}$. If $C_{3}^{*}$ is contained in $X \cup e$, then $e \in \operatorname{cl}(X) \cap \mathrm{cl}^{*}(X)$, so $|E(M)| \leq 8$; a contradiction. It follows, by orthogonality, that $C_{3}^{*}=\left\{e, d_{2}, c_{2}, h\right\}$ for some $h \in E(M)-(X \cup e)$. Since $M \backslash d_{2} \backslash e / c$ has an $N$-minor, and $\left\{c_{2}, h\right\}$ is a series pair in this matroid, $M / c / h$ has an $N$-minor. Therefore, $\{h, c\}$ is contained in a 4-element circuit. As $\{h, c\}$ meets both $C_{3}^{*}$ and $Q_{1}$, and these cocircuits are disjoint, we see that $h \in \operatorname{cl}(X \cup e)$, by orthogonality. But as $X \cup e$ is 3-separating and $h \in \mathrm{cl}^{*}(X \cup e)$, this implies $|E(M)|=9$. Then $E(M)-X$ is a triangle containing $h$, but $M / h$ has an $N$-minor, so this triangle is not $N$-grounded; a contradiction. Hence we may assume that $C_{i} \subseteq X$ for $i \in\{1,2\}$.

We will show that $X$ is an elongated-quad 3-separator, labelled as illustrated in Figure 5. By 4.3.1, $c_{2} \notin \operatorname{cl}\left(Q_{1}\right)$, so $d_{2} \in C_{1}$ and $d_{2} \in C_{2}$. As $C_{1}^{*}$ is independent, $d_{0} \notin C_{2}$, so $C_{2}=\left\{c_{2}, x, d_{2}, q\right\}$ for $q \in\left\{c_{1}, d_{1}\right\}$. Suppose $q=c_{1}$. Then, as $M \backslash d_{0} \backslash d_{1} / c_{1} / c_{2}$ has an $N$-minor, and $\left\{x, d_{2}\right\}$ is a parallel pair in this matroid, $M \backslash d_{0} \backslash d_{1} \backslash x$ has an $N$-minor. Since $\left\{c_{1}, c_{2}, d_{2}\right\}$ is a series class in $M \backslash d_{0} \backslash d_{1} \backslash x$, the matroid $M / c_{2} / d_{2}$ has an $N$-minor. Observe that $Q_{1}$ is 3 -separating in $M / c_{2}$, and $d_{2} \in \mathrm{cl}_{M / c_{2}}\left(Q_{1}\right)$. It follows that (i) holds by the dual of Lemma 4.2, So we may assume that $C_{2}=\left\{c_{2}, x, d_{1}, d_{2}\right\}$. Recall that $\left\{c_{1}, c_{2}, d_{2}\right\} \subseteq C_{1} \subseteq X$. If $d_{0} \notin C_{1}$, then, due to the circuits $Q_{1}$ and $C_{2}$, 
the set $\left\{c_{2}, x, d_{2}\right\}$ spans $X$, so $r(X) \leq 3$; a contradiction. We deduce that $C_{1}=\left\{c_{1}, c_{2}, d_{2}, d_{0}\right\}$. Finally, as $C_{2}^{*}=\left\{d_{1}, d_{2}, c_{1}, c_{2}\right\}$ is a cocircuit, $X$ is an elongated-quad 3-separator.

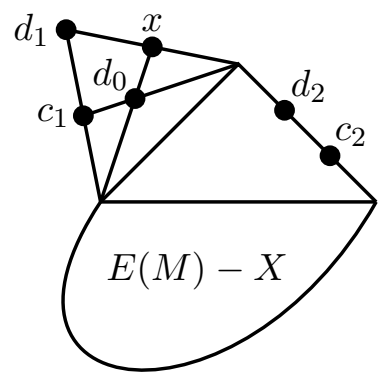

FiguRE 5. The labelling of the elongated-quad 3-separator in 4.3.7, where $\left\{d_{0}, d_{1}, d_{2}\right\} \subseteq D$ and $\left\{c_{1}, c_{2}\right\} \subseteq C$.

Suppose that $E(M)-E(N) \nsubseteq X$. It remains to show that $|E(M)|=10$ and (iv) holds. Let $(C, D)$ be an $N$-labelling of $M$ with $\left\{c_{1}, c_{2}\right\} \subseteq C$ and $\left\{d_{0}, d_{1}, d_{2}\right\} \subseteq D$, and observe that there exists either some $c^{\prime} \in C-X$ or some $d^{\prime} \in D-X$.

First, we claim that if there is some such $c^{\prime}$, and $C_{3}$ is a 4-element circuit containing $c^{\prime}$ and meeting $X$, then $C_{3} \cap X \in$ $\left\{\left\{x, d_{0}\right\},\left\{c_{1}, d_{1}\right\},\left\{c_{2}, d_{2}\right\},\left\{d_{0}, d_{1}, d_{2}\right\},\left\{d_{0}, d_{1}, c_{2}\right\}\right\}$. Let $C_{3}$ be such a circuit. By orthogonality with $Q_{1}, C_{1}^{*}$ and $C_{2}^{*}$, either $C_{3} \cap X$ is one of $\left\{x, d_{0}\right\}$, $\left\{c_{1}, d_{1}\right\}$, or $\left\{c_{2}, d_{2}\right\}$; or $C_{3}$ has one element from each of these three sets. Suppose the latter. If $\left|C_{3} \cap C\right| \geq 3$, then, after an $N$-label switch, one of $x$, $c_{1}$, or $c_{2}$ is $N$-labelled for deletion, while retaining that $d_{0}, d_{1}$, or $d_{2}$ is $N$ labelled for deletion, respectively. That is, one of $\left\{x, d_{0}\right\},\left\{c_{1}, d_{1}\right\}$, or $\left\{c_{2}, d_{2}\right\}$ is $N$-labelled for deletion, and it follows that (i) holds by Lemma 4.2. So $\left|C_{3} \cap C\right| \leq 2$. Then $C_{3}$ contains at least two of $\left\{d_{0}, d_{1}, d_{2}\right\}$. If $\left\{d_{0}, d_{1}\right\} \nsubseteq C_{3}$, then either $C_{3}=\left\{x, d_{1}, d_{2}, c^{\prime}\right\}$ or $C_{3}=\left\{d_{0}, c_{1}, d_{2}, c^{\prime}\right\}$; so either $C_{2} \cup c^{\prime}$ or $C_{1} \cup c^{\prime}$ is a 5-element plane intersecting $Q_{1}$ in two elements, respectively, which contradicts orthogonality. So $C_{3} \cap X \in\left\{\left\{d_{0}, d_{1}, c_{2}\right\},\left\{d_{0}, d_{1}, d_{2}\right\}\right\}$, thus proving the claim.

By a dual argument if there exists some $d^{\prime} \in D-X$, and $C_{3}^{*}$ is a 4-element cocircuit containing $d^{\prime}$ and meeting $X$, then $C_{3}^{*} \cap X \in$ $\left\{\left\{x, d_{1}\right\},\left\{c_{1}, d_{0}\right\},\left\{c_{2}, d_{2}\right\},\left\{x, c_{1}, c_{2}\right\},\left\{x, c_{1}, d_{2}\right\}\right\}$.

Suppose there exists some $d^{\prime} \in D-X$. Then $d^{\prime}$ is contained in a 4-element cocircuit with $d_{1}$, and hence $x$. Let this cocircuit be $\left\{d^{\prime}, d_{1}, x, c^{\prime}\right\}$ for some $c^{\prime} \in E(M)-\left(X-d^{\prime}\right)$. Up to an $N$-label switch on $x$ and $c^{\prime}$, the element $c^{\prime}$ is $N$-labelled for contraction. So we may assume that there is some $c^{\prime} \in C-X$.

Let $C_{3}$ be the 4-element circuit containing $\left\{c^{\prime}, c_{1}\right\}$. Then $C_{3} \cap X=$ $\left\{c_{1}, d_{1}\right\}$. So let $C_{3}=\left\{c^{\prime}, c_{1}, d_{1}, z_{1}\right\}$ for some $z_{1} \in E(M)-\left(X \cup c^{\prime}\right)$. By an $N$-label switch on $d_{1}$ and $z_{1}$, the element $z_{1}$ is $N$-labelled for deletion. 
So $\left\{z_{1}, d_{2}\right\}$ is contained in a 4 -element cocircuit $C_{3}^{*}$. Either $C_{3}^{*}=$ $\left\{z_{1}, d_{2}, c_{1}, x\right\}$, or $C_{3}^{*} \cap X=\left\{c_{2}, d_{2}\right\}$. In the latter case, it follows, by orthogonality with $C_{3}$ and $Q_{1}$, that $C_{3}^{*}=\left\{z_{1}, c_{2}, d_{2}, c^{\prime}\right\}$. Thus, in either case, $r^{*}\left(X \cup\left\{c^{\prime}, z_{1}\right\}\right) \leq r^{*}(X)+1=5$.

Now if $c^{\prime} \in \operatorname{cl}(X)$, then $r\left(X \cup\left\{c^{\prime}, z_{1}\right\}\right)=r(X)=4$, so $\lambda\left(X \cup\left\{c^{\prime}, z\right\}\right) \leq$ $4+5-8=1$. It follows that $|E(M)|=9$, and hence $E(M)-X$ is a triangle containing the $N$-contractible element $c^{\prime}$; a contradiction. Thus the 4-element circuit $C_{4}$ containing $\left\{c^{\prime}, c_{2}\right\}$ is not contained in $X \cup c^{\prime}$. Hence $C_{4}=\left\{c^{\prime}, c_{2}, d_{2}, z_{2}\right\}$ for some $z_{2} \in E(M)-\left(X \cup c^{\prime}\right)$. If $z_{1}=z_{2}$, then $\left\{c^{\prime}, z_{1}, c_{1}, c_{2}\right\}$ spans $X \cup\left\{c^{\prime}, z_{1}\right\}$, so $\lambda\left(X \cup\left\{c^{\prime}, z_{1}\right\}\right) \leq 4+5-8=1$; as before, this is contradictory. So $z_{1} \neq z_{2}$.

Let $C_{4}^{*}$ be the 4-element cocircuit containing $\left\{z_{2}, d_{1}\right\}$. Then $C_{4}^{*} \cap X=$ $\left\{d_{1}, x\right\}$. By orthogonality with $C_{3}$ and $C_{4}$, we see that $C_{4}^{*}=\left\{z_{2}, d_{1}, x, c^{\prime}\right\}$, so $z_{2} \in \mathrm{cl}^{*}\left(X \cup c^{\prime}\right)$. Now $r^{*}\left(X \cup\left\{c^{\prime}, z_{1}, z_{2}\right\}\right) \leq 5$, so $\lambda\left(X \cup\left\{c^{\prime}, z_{1}, z_{2}\right\}\right) \leq$ $5+5-9=1$. Hence $|E(M)|=10$. Let $Q_{2}=E(M)-\left(Q_{1} \cup\left\{c_{2}, d_{2}\right\}\right)$ and pick $q$ so that $Q_{2}=\left\{q, c^{\prime}, z_{1}, z_{2}\right\}$. It is now easily checked that $C_{3},\left\{d_{0}, x, c^{\prime}, z_{1}\right\}$, $\left\{c_{1}, d_{1}, z_{2}, q\right\}$, and $\left\{d_{0}, x, z_{2}, q\right\}$ are circuits; $C_{4}^{*},\left\{d_{0}, c_{1}, z_{2}, c^{\prime}\right\},\left\{d_{1}, x, q, z_{1}\right\}$, and $\left\{d_{0}, c_{1}, q, z_{1}\right\}$ are cocircuits; and $Q_{1}$ and $\left\{q, c^{\prime}, z_{1}, z_{2}\right\}$ are quads. So $Q_{1} \cup Q_{2}$ is a double-quad 3-separator. Moreover, $C_{4}$ and $\left\{c_{2}, d_{2}, z_{1}, q\right\}$ are circuits, and $C_{3}^{*}=\left\{c_{2}, d_{2}, z_{1}, c^{\prime}\right\}$ and $\left\{c_{2}, d_{2}, z_{2}, q\right\}$ are cocircuits; so $Q_{2} \cup$ $\left\{c_{2}, d_{2}\right\}$ is an elongated-quad 3 -separator. Thus (iv) holds. This completes the proof of 4.3 .7 .

The proof now follows from 4.3.3 4.3.7

Lemma 4.4. Suppose that for every $N$-labelling $\left(C^{\prime}, D^{\prime}\right)$ that is switchingequivalent to $(C, D)$, and for every pair $\{x, y\} \subseteq C^{\prime}$ or $\{x, y\} \subseteq D^{\prime}$, the pair $\{x, y\}$ is not contained in a quad. Then, up to replacing $(M, N)$ by $\left(M^{*}, N^{*}\right)$, either

(i) there is some $X \subseteq E(M)$ such that $E(M)-E(N) \subseteq X$ and $X$ is a skew-whiff 3-separator;

(ii) there exists $d \in E(M)$ such that $M \backslash d$ is 3-connected and has a cyclic 3-separation $\left(Y,\left\{d^{\prime}\right\}, Z\right)$ with $|Y| \geq 4$, where $M \backslash d \backslash d^{\prime}$ has an $N$ minor with $|Y \cap E(N)| \leq 1$;

(iii) $M$ is a nest of twisted cubes; or

(iv) $|E(M)| \leq 10$.

Proof. Suppose that neither (ii) nor (iii) holds; we will show that (i) or (iv) holds. Again, we will abuse notation by using $(C, D)$ to refer to an $N$-labelling that is switching-equivalent to $(C, D)$.

4.4.1. Let $C_{0}$ be a 4-element circuit containing at least two elements in $C \cup D$, and suppose there are distinct elements $d^{\prime}, d^{\prime \prime} \in D \cap\left(\operatorname{cl}^{*}\left(C_{0}\right)-C_{0}\right)$. Then (ii) holds.

Subproof. Since $C_{0}$ is not a quad, it is coindependent. Hence $C_{0}$ is 3separating in the 3-connected matroid $M \backslash d^{\prime}$, and $d^{\prime \prime} \in \mathrm{cl}_{M \backslash d^{\prime}}^{*}\left(C_{0}\right)$. It now follows from Lemma 4.2 that (ii) holds. 
Since $|E(M)|-|E(N)| \geq 5$, up to duality we may assume that $|D| \geq 3$. Let $\left\{d_{0}, d_{1}, d_{2}\right\} \subseteq D$. Then there is a 4-element cocircuit $C_{1}^{*}$ containing $\left\{d_{0}, d_{1}\right\}$, and a 4 -element cocircuit $C_{2}^{*}$ containing $\left\{d_{0}, d_{2}\right\}$. We start by showing that if $C_{1}^{*} \neq C_{2}^{*}$, then $r^{*}\left(C_{1}^{*} \cup C_{2}^{*}\right) \geq 4$. More generally, we prove the following:

4.4.2. Let $d, d^{\prime}$, and $d^{\prime \prime}$ be distinct elements in $D$, and suppose $\left\{d, d^{\prime}\right\}$ is contained in a 4-element cocircuit $C^{*}$, and $\left\{d, d^{\prime \prime}\right\}$ is contained in a 4-element cocircuit $C_{0}^{*}$, where $C^{*} \neq C_{0}^{*}$. Then $r^{*}\left(C^{*} \cup C_{0}^{*}\right) \geq 4$.

Subproof. Let $X=C^{*} \cup C_{0}^{*}$, and suppose $r^{*}(X)=3$. Note that $|X| \geq 5$. Since each $x \in X-\left\{d, d^{\prime}, d^{\prime \prime}\right\}$ is a coloop in $M \backslash d \backslash d^{\prime} \backslash d^{\prime \prime}$, and this matroid has an $N$-minor, every pair contained in $X$ is $N$-deletable. As each $x \in X$ is not contained in a triad, $X$ is a coplane. Now, for distinct $d_{0}, d_{0}^{\prime} \in X$, the set $\mathrm{cl}^{*}(X)-\left\{d_{0}, d_{0}^{\prime}\right\}$ is a series class in $M \backslash d_{0} \backslash d_{0}^{\prime}$, so each pair $\left\{c_{1}, c_{2}\right\} \subseteq X$ is $N$-contractible. Note that since $M$ is 3-connected, $r(X) \geq|X|-1$. Thus $X$ contains at most one circuit. Let $\left\{c, c^{\prime}\right\}$ be a pair of elements of $X$ not contained in such a circuit. Up to switching $N$-labels, we may assume that the pair $\left\{c, c^{\prime}\right\}$ is $N$-labelled for contraction, and the elements in $X-\left\{c, c^{\prime}\right\}$ are $N$-labelled for deletion. Thus $\left\{c, c^{\prime}\right\}$ is contained in a circuit $C_{0}$, and this circuit is not contained in $X$. By orthogonality, $\left|C_{0} \cap X\right|=3$, and hence $C_{0}$ cospans $X$. Now $X-C_{0}$ contains a pair that is $N$-labelled for deletion, and is contained in $\operatorname{cl}^{*}\left(C_{0}\right)-C_{0}$. So (ii) holds by 4.4.1 a contradiction. We deduce that $r^{*}(X) \geq 4$.

Next we show that we may assume that $d_{2} \notin C_{1}^{*}$. Suppose $D \subseteq C_{1}^{*}$. Then $C_{1}^{*}=\left\{c, d_{0}, d_{1}, d_{2}\right\}$ for some $c \in C$. Since $|E(M)|-|E(N)| \geq 5$, there exists some $c^{\prime} \in C-C_{1}^{*}$, and $\left\{c, c^{\prime}\right\}$ is contained in a 4-element circuit $C_{1}$. By orthogonality, $\left|C_{1} \cap C_{1}^{*}\right| \geq 2$. Suppose $\left|C_{1} \cap C_{1}^{*}\right|=3$. Without loss of generality, let $C_{1}=\left\{c, c^{\prime}, d_{1}, d_{2}\right\}$. Then we can swap the $N$-labels on $c$ and $d_{0}$ to deduce that there is a 4 -element circuit $C_{2}$ containing $\left\{c^{\prime}, d_{0}\right\}$. If $C_{2}$ is contained in $C_{1} \cup d_{0}$, then $r\left(C_{1}^{*}\right)=3$, so $C_{1}^{*}$ is a quad; a contradiction. We deduce, by orthogonality, that $\left|C_{2} \cap C_{1}^{*}\right|=2$. We may now assume, up to relabelling and an $N$-label switch, that $\left|C_{1} \cap C_{1}^{*}\right|=2$. Without loss of generality, let $C_{1}=\left\{x, c, c^{\prime}, d_{2}\right\}$, where $x \in E(M)-\left(C_{1}^{*} \cup c^{\prime}\right)$. Now, by switching the $N$-labels on $x$ and $d_{2}$, we obtain an element $x \in D-C_{1}^{*}$.

We may now assume that $d_{2} \notin C_{1}^{*}$. Let $X=C_{1}^{*} \cup C_{2}^{*}$. By 4.4.2, $r^{*}(X) \geq 4$, so $\left|C_{1}^{*} \cap C_{2}^{*}\right| \leq 2$. We work towards 4.4.8, which handles the case where $\left|C_{1}^{*} \cap C_{2}^{*}\right|=2$. First, in 4.4 .3 , we consider the case where $\left|C_{1}^{*} \cap C_{2}^{*}\right|=2$ and $\lambda(X)=2$.

4.4.3. Suppose $\left|C_{1}^{*} \cap C_{2}^{*}\right|=2$. If $X$ is 3-separating, then $X$ is a skew-whiff 3-separator of $M$ with $E(M)-E(N) \subseteq X$.

Subproof. Observe that $r^{*}(X)=4$, by 4.4 .2 and since $\left|C_{1}^{*} \cap C_{2}^{*}\right|=2$. As $\lambda(X)=2$ and $|X|=6$, it follows that $r(X)=4$. We claim that $d_{1} \notin C_{2}^{*}$. Suppose $d_{1} \in C_{2}^{*}$. Then $C_{1}^{*} \cap C_{2}^{*}=\left\{d_{0}, d_{1}\right\}$, so let $C_{1}^{*}-C_{2}^{*}=\left\{c_{1}, c_{2}\right\}$. Since $M \backslash d_{0} \backslash d_{1} \backslash d_{2}$ has an $N$-minor, $\left\{c_{1}, c_{2}\right\}$ is $N$-contractible. Now $\left\{c_{1}, c_{2}\right\} \subseteq$ 
$\operatorname{cl}\left(C_{2}^{*}\right)-C_{2}^{*}$, since $C_{2}^{*}$ is independent and $r(X)=4$. So (ii) holds by the dual of 4.4.1; a contradiction. This proves the claim.

So let $C_{1}^{*}=\left\{d_{1}, c_{1}, d_{0}, x\right\}$ and $C_{2}^{*}=\left\{d_{2}, c_{2}, d_{0}, x\right\}$ for distinct $c_{1}, c_{2}, x \in$ $E(M)-\left\{d_{0}, d_{1}, d_{2}\right\}$. Since the pair $\left\{c_{1}, c_{2}\right\}$ is $N$-contractible, it is contained in a 4-element circuit $C_{0}$.

To begin with, we work under the assumption that no 4-element circuit containing $\left\{c_{1}, c_{2}\right\}$ is contained in $X$. Then, by orthogonality, $C_{0}=$ $\left\{c_{1}, c_{2}, x^{\prime}, e\right\}$, where $x^{\prime} \in\left\{d_{0}, x\right\}$, and $e \in E(M)-X$. Observe that $(X, E(M)-X)$ is a 3 -separation, and $e \in \operatorname{cl}(X)-X$, so $e \notin \mathrm{cl}^{*}(X)$. Since $\left\{x^{\prime}, e\right\}$ is a parallel pair in $M / c_{1} / c_{2}$, by possibly swapping the $N$-labels on $x^{\prime}$ and $e$ we deduce that the pairs $\left\{e, d_{1}\right\}$ and $\left\{e, d_{2}\right\}$ are $N$-deletable. These pairs are contained in 4-element cocircuits $C_{3}^{*}$ and $C_{4}^{*}$ respectively. Neither of these cocircuits is contained in $X \cup e$, otherwise $e \in \mathrm{cl}^{*}(X)$; a contradiction. Moreover, each of these cocircuits meets $C_{0}$, so they do so in at least two elements, by orthogonality.

Since $C_{1}^{*}$ and $C_{2}^{*}$ are independent and $r(X)=4$, the element $c_{2}$ is in a circuit $C_{2}$ contained in $C_{1}^{*} \cup c_{2}$, and $c_{1}$ is in a circuit $C_{1}$ contained in $C_{2}^{*} \cup c_{1}$. The circuit $C_{1}$ (or $C_{2}$ ) can only intersect $C_{3}^{*}$ (or $C_{4}^{*}$, respectively) in at most one element, so, by orthogonality, $C_{3}^{*} \cap C_{1}=\emptyset$ and $C_{4}^{*} \cap C_{2}=\emptyset$. In particular, $c_{1} \notin C_{3}^{*}$ and $c_{2} \notin C_{4}^{*}$. Since $X$ does not contain any triangles, $C_{1}=X-C_{3}^{*}$ and $C_{2}=X-C_{4}^{*}$. If $x^{\prime} \in C_{3}^{*} \cap C_{4}^{*}$, then $C_{3}^{*} \cap C_{1}=\left\{d_{1}\right\}$; a contradiction to orthogonality. Without loss of generality, we may now assume that $x^{\prime} \notin C_{4}^{*}$, in which case $c_{1} \in C_{4}^{*}$.

Suppose $x^{\prime} \in C_{3}^{*}$. Let $C_{3}^{*}-\left\{e, d_{1}, x^{\prime}\right\}=\{f\}$. Since $M \backslash d_{1} \backslash d_{2} / c_{1} / c_{2}$ has an $N$-minor, and $\left\{e, x^{\prime}\right\}$ is a parallel pair in this matroid, $M \backslash d_{1} \backslash e / c_{2}$ has an $N$-minor, implying $M / c_{2} / f$ has an $N$-minor. So $\left\{c_{2}, f\right\}$ is contained in a 4-element circuit $C_{2}^{\prime}$. Observe that $C_{2}^{\prime}$ is not contained in $X \cup\{e, f\}$, for otherwise $f \in \operatorname{cl}(X \cup e) \cap \mathrm{cl}^{*}(X \cup e)$, so $\lambda(X \cup\{e, f\}) \leq 1$ and $|E(M)| \leq 9$; a contradiction. Now, by orthogonality, $C_{2}^{\prime}$ meets $C_{2}^{*}-c_{2}$ and $C_{3}^{*}-f$. As the intersection of these last two sets is $\left\{x^{\prime}\right\}$, we deduce $x^{\prime} \in C_{2}^{\prime}$. But then $C_{2}^{\prime}$ also has an element in $C_{1}^{*}-x^{\prime}$, by orthogonality; a contradiction. So $x^{\prime} \notin C_{3}^{*}$, hence $c_{2} \in C_{3}^{*}$. Now $C_{3}^{*}=\left\{e, d_{1}, c_{2}, f\right\}$ and $C_{4}^{*}=\left\{e, d_{2}, c_{1}, f^{\prime}\right\}$ for some $f, f^{\prime} \in E(M)-(X \cup e)$, and $C_{1}=\left\{x, d_{0}, c_{1}, d_{2}\right\}$ and $C_{2}=\left\{x, d_{0}, c_{2}, d_{1}\right\}$.

Recall that $C_{0}=\left\{c_{1}, c_{2}, x^{\prime}, e\right\}$, where $x^{\prime} \in\left\{d_{0}, x\right\}$. If $x^{\prime}=x$, then $M \backslash d_{0} \backslash d_{1} \backslash d_{2} / c_{1} / c_{2}$ has an $N$-minor and $\{x, e\}$ is a parallel pair in this matroid, so $M \backslash d_{0} \backslash d_{1} \backslash d_{2} \backslash x$ has an $N$-minor. But $c_{2}$ and $c_{1}$ are coloops in this matroid, so we may assume that $X \subseteq D$, in which case there is a pair of $N$-deletable elements in $\mathrm{cl}^{*}\left(C_{2}\right)-C_{2}$, so (ii) holds by 4.4.1. Hence $x^{\prime}=d_{0}$.

The pair $\left\{d_{1}, d_{2}\right\}$ is also contained in a 4 -element cocircuit $C_{5}^{*}$. By orthogonality with the circuits $C_{1}$ and $C_{2}$, either $C_{5}^{*}=C_{1}^{*} \triangle C_{2}^{*}$, or $C_{5}^{*}$ meets $C_{1}^{*} \cap C_{2}^{*}=\left\{x, d_{0}\right\}$.

We start with the former case. Recall that $C_{3}^{*}=\left\{e, d_{1}, c_{2}, f\right\}$ and $C_{4}^{*}=$ $\left\{e, d_{2}, c_{1}, f^{\prime}\right\}$. Recall also that $M \backslash d_{1} \backslash d_{2} \backslash e$ has an $N$-minor, and observe that $\left\{c_{1}, c_{2}\right\}$ and $\left\{c_{1}, f^{\prime}\right\}$ are both series pairs in this matroid, due to the cocircuits $C_{5}^{*}$ and $C_{4}^{*}$. Now $\left\{c_{1}, f^{\prime}\right\}$ is $N$-contractible, so this pair is contained in a 
4-element circuit $C_{1}^{\prime}$. This circuit is not contained in $X \cup\left\{e, f^{\prime}\right\}$, otherwise $f^{\prime} \in \operatorname{cl}(X \cup e)$ in which case $|E(M)|=9$. By orthogonality, $C_{1}^{\prime}$ meets $C_{1}^{*}-c_{1}=\left\{d_{1}, x, d_{0}\right\}$, and $C_{5}^{*}-c_{1}=\left\{d_{1}, d_{2}, c_{2}\right\}$. So $d_{1} \in C_{1}^{\prime}$. Now, by orthogonality with $C_{3}^{*}$, we see that $C_{1}^{\prime}$ contains an element in $\left\{e, c_{2}, f\right\}$. If $f \notin C_{1}^{\prime}$, then $f^{\prime} \in \operatorname{cl}(X \cup e)$; a contradiction. Suppose $f \neq f^{\prime}$. Then $C_{1}^{\prime}=\left\{c_{1}, f^{\prime}, d_{1}, f\right\}$. Recall that the pair $\left\{c_{1}, f^{\prime}\right\}$ is $N$-contractible in $M \backslash e$. As $\left\{d_{1}, f\right\}$ is a parallel pair in $M \backslash e / c_{1} / f^{\prime}$, the matroid $M \backslash e \backslash f / c_{1}$ has an $N$ minor. In turn, we see that $\left\{d_{1}, c_{2}\right\}$ is a series pair in this matroid, due to the cocircuit $C_{3}^{*}$, so the pair $\left\{c_{1}, d_{1}\right\}$ is $N$-contractible. As $\left\{c_{1}, d_{1}\right\} \subseteq \operatorname{cl}\left(C_{2}^{*}\right)-C_{2}^{*}$, it follows that (ii) holds, by 4.4.1. Now $f=f^{\prime}$, and $C_{1}^{\prime}=\left\{c_{1}, d_{1}, f, g\right\}$ for some $g \in E(M)-(X \cup\{e, f\})$. Since $M \backslash e / c_{1} / f$ has an $N$-minor, and $\left\{d_{1}, g\right\}$ is a parallel pair in this matroid, $\{e, g\}$ is contained in a 4element cocircuit. By orthogonality with $C_{0}, C_{1}$, and $C_{2}$, this cocircuit is contained in $X \cup\{e, g\}$. Hence $g \in \operatorname{cl}(X \cup\{e, f\}) \cap \mathrm{cl}^{*}(X \cup\{e, f\})$, implying $\lambda(X \cup\{e, f, g\}) \leq 1$, so $|E(M)| \in\{9,10\}$; a contradiction.

So we may assume that $C_{5}^{*}$ meets $\left\{d_{0}, x\right\}$. Suppose $d_{0} \in C_{5}^{*}$. Then, by orthogonality with $C_{0}$, the cocircuit $C_{5}^{*}$ meets $\left\{c_{1}, c_{2}, e\right\}$. If $C_{5}^{*}$ meets $\left\{c_{1}, c_{2}\right\}$, it follows that $r^{*}(X)=3$; on the other hand, if $e \in C_{5}^{*}$, then $e \in \operatorname{cl}^{*}(X)$; either case is contradictory. We deduce that $x \in C_{5}^{*}$. Let $C_{5}^{*}=\left\{d_{1}, d_{2}, x, f\right\}$. Since $M \backslash d_{1} \backslash d_{2} / c_{1} / c_{2}$ has an $N$-minor, and in this matroid $\{x, f\}$ is a series pair and $\left\{e, d_{0}\right\}$ is a parallel pair, we see that $M / c_{1} / x \backslash e$ has an $N$-minor. Now $\left\{d_{0}, d_{2}\right\}$ is a parallel pair in this matroid, due to the circuit $C_{1}$, so $M \backslash e \backslash d_{0}$ has an $N$-minor. Hence $\left\{e, d_{0}\right\}$ is contained in a 4-element cocircuit $C_{6}^{*}$. This cocircuit cannot be contained in $X \cup e$, as $e \notin \mathrm{cl}^{*}(X)$. By orthogonality with the circuits $C_{1}$ and $C_{2}$, we deduce that $x \in C_{6}^{*}$. However, by circuit elimination on $C_{1}$ and $C_{2}$, the set $X-x$ contains a circuit. By orthogonality with $C_{6}^{*}$, we deduce that $C_{1}^{*} \triangle C_{2}^{*}$ is a circuit. But this contradicts our assumption that no 4-element circuit containing $\left\{c_{1}, c_{2}\right\}$ is contained in $X$.

Now we may assume that $\left\{c_{1}, c_{2}\right\}$ is contained in a 4-element circuit $C_{0} \subseteq$ $X$. Since $C_{0}$ is not a quad, $C_{0}$ is coindependent. If $x \in C_{0}$, then $X-C_{0}$ is a pair that is $N$-labelled for deletion, and in the coclosure of the circuit $C_{0}$, in which case (ii) holds by 4.4.1. So we may assume that $x \notin C_{0}$. Now, either $C_{0}=C_{1}^{*} \triangle C_{2}^{*}$, or we may assume, by symmetry (that is, up to swapping $d_{1}$ and $\left.d_{2}\right)$, that $C_{0}=\left\{c_{1}, d_{0}, c_{2}, d_{2}\right\}$.

Suppose we are in the former case, where $C_{0}=C_{1}^{*} \triangle C_{2}^{*}$ is a circuit. Suppose $\left\{c_{1}, c_{2}, x, d_{0}\right\}$ is a circuit. Then $\left\{d_{1}, d_{2}\right\} \subseteq \mathrm{cl}^{*}\left(\left\{c_{1}, c_{2}, x, d_{0}\right\}\right)$, and the pair $\left\{d_{1}, d_{2}\right\}$ is $N$-labelled for deletion. Thus (ii) holds by 4.4.1, So $\left\{c_{1}, c_{2}, x, d_{0}\right\}$ is not a circuit.

Next, we claim that $X-x$ and $X-d_{0}$ are cocircuits. Certainly, as $C_{0}$ is coindependent and cospans $X$, each of the sets $X-x$ and $X-d_{0}$ contains a cocircuit, and these cocircuits contain $d_{0}$ and $x$, respectively. If either of these sets properly contains a cocircuit, it follows that $r^{*}(X)=3$; a contradiction. So $X-x$ and $X-d_{0}$ are cocircuits, as claimed. 
Let $C_{0}^{*}$ be the 4-element cocircuit that contains $\left\{d_{1}, d_{2}\right\}$. As $C_{2}^{*} \cup c_{1}$ contains a circuit, and this circuit is not $\left\{c_{1}, c_{2}, x, d_{0}\right\}$, the circuit must contain $d_{2}$. So $C_{0}^{*}$ meets $X-\left\{d_{1}, d_{2}\right\}$, by orthogonality. Now, if $C_{0}^{*} \subseteq X$, then $\left|C_{0}^{*}-C_{1}^{*}\right|>1$ and $\left|C_{0}^{*}-C_{2}^{*}\right|>1$, otherwise $r^{*}(X)=3$. Moreover, $C_{0}^{*} \neq C_{0}$, since $C_{0}$ is coindependent. So $C_{0}^{*} \nsubseteq X$; hence $\left|C_{0}^{*} \cap X\right|=3$. Let $C_{0}^{*}-X=\{f\}$.

Since $f \in \operatorname{cl}^{*}(X)$ and $|E(M)-(X \cup f)| \geq 2$, the set $X \cup f$ is exactly 3separating. So $f \notin \operatorname{cl}(X)$. In $M \backslash d_{0} \backslash d_{1} \backslash d_{2}$, the set $\left\{c_{1}, c_{2}, x, f\right\}$ is contained in a series class. So $\{f, x\}$ is $N$-contractible, and thus is contained in a 4 element circuit $C_{0}^{\prime}$. By orthogonality with the cocircuits $C_{1}^{*}$ and $C_{2}^{*}$, either $C_{0}^{\prime} \subseteq X \cup f$ or $d_{0} \in C_{0}^{\prime}$. Since $f \notin \operatorname{cl}(X)$, we deduce that $d_{0} \in C_{0}^{\prime}$. But now $\left\{f, x, d_{0}\right\}$ meets the cocircuit $X-x$, so, by orthogonality, $C_{0}^{\prime}$ also meets $X-\left\{x, d_{0}\right\}$, implying $f \in \operatorname{cl}(X)$; a contradiction.

Now, suppose that $C_{0}=\left\{c_{1}, d_{0}, c_{2}, d_{2}\right\}$. Since $\left\{d_{1}, d_{2}\right\}$ is $N$-labelled for deletion, $\left\{d_{1}, d_{2}\right\}$ is contained in a 4 -element cocircuit $C_{0}^{*}$ that, by orthogonality, intersects $C_{0}$ in at least two elements. Suppose $C_{0}^{*}$ is not contained in $X$. If $d_{0} \in C_{0}^{*}$, then $\left\{d_{1}, c_{1}\right\}$ is an $N$-contractible pair in the closure of the independent cocircuit $C_{2}^{*}$, so (ii) holds by the dual of 4.4.1. So $C_{0}^{*}=\left\{d_{1}, d_{2}, c^{\prime}, f\right\}$ for some $c^{\prime} \in\left\{c_{1}, c_{2}\right\}$ and $f \in E(M)-X$. Since $\left\{c_{1}, c_{2}, x, f\right\}$ is contained in a series class in $M \backslash d_{0} \backslash d_{1} \backslash d_{2}$, the pair $\{f, x\}$ is contained in a circuit $C_{0}^{\prime}$. Since $X$ and $X \cup f$ are exactly 3separating, and $f \in \mathrm{cl}^{*}(X)$, we have $f \notin \operatorname{cl}(X)$. Hence, the circuit $C_{0}^{\prime}$ is not contained in $X \cup f$. It follows, by orthogonality, that $d_{0} \in C_{0}^{\prime}$, and $C_{0}^{\prime} \cap X=\left\{x, d_{0}\right\}$. But then we get a contradiction to orthogonality using the cocircuit $C_{0}^{*}=\left\{d_{1}, d_{2}, c^{\prime}, f\right\}$. So $C_{0}^{*} \subseteq X$. Since $r^{*}(X)=4$, it follows that $C_{0}^{*}=C_{1}^{*} \triangle C_{2}^{*}$.

Let $C_{1}$ and $C_{2}$ be the 4-element circuits containing $\left\{c_{1}, x\right\}$, and $\left\{c_{2}, x\right\}$ respectively. By orthogonality with $C_{0}^{*}$ and $C_{1}^{*}$, and since $C_{2} \neq C_{2}^{*}$ as $C_{2}^{*}$ is independent, $C_{2}$ meets $\left\{c_{1}, d_{1}\right\}$. Note also that $C_{2} \neq\left\{c_{1}, c_{2}, x, d_{1}\right\}$, for otherwise the pair $\left\{d_{0}, d_{2}\right\}$ is $N$-labelled for deletion and contained in $\operatorname{cl}^{*}\left(\left\{c_{1}, c_{2}, x, d_{1}\right\}\right)$, so that (ii) holds by 4.4.1. Now, if $c_{1} \in C_{2}$, then, as $M \backslash d_{1} / c_{1} / c_{2}$ has an $N$-minor, $\left\{d_{1}, x\right\}$ is $N$-deletable. As $\left\{d_{1}, x\right\} \subseteq \mathrm{cl}^{*}\left(C_{0}\right)-$ $C_{0}$, it follows that (ii) holds, by 4.4.1, a contradiction. So $d_{1} \in C_{2}$. By a similar argument with $C_{1}$, we deduce that $C_{1}$ meets $\left\{c_{2}, d_{2}\right\}$, and $c_{2} \notin C_{1}$, so $d_{2} \in C_{1}$.

Suppose $C_{2} \nsubseteq X$. Let $C_{2}=\left\{c_{2}, x, d_{1}, e\right\}$, where $e \in E(M)-X$. Since $M \backslash d_{0} \backslash d_{2} / c_{2} / x$ has an $N$-minor, and $\left\{d_{1}, e\right\}$ is a parallel pair in this matroid, $\left\{d_{0}, d_{2}, e\right\}$ is $N$-deletable. Now $\left\{d_{0}, e\right\}$ and $\left\{d_{2}, e\right\}$ are contained in 4-element cocircuits $C_{3}^{*}$ and $C_{4}^{*}$ respectively. If $C_{3}^{*}$ or $C_{4}^{*}$ is contained in $X \cup e$, then $e \in \operatorname{cl}^{*}(X) \cap \operatorname{cl}(X)$; a contradiction. So $C_{3}^{*}$ and $C_{4}^{*}$ each contain some element in $E(M)-(X \cup e)$. By orthogonality with $C_{0}$ and $C_{2}$, the cocircuit $C_{3}^{*}$ meets $\left\{c_{1}, c_{2}, d_{2}\right\}$ and $\left\{c_{2}, x, d_{1}\right\} ;$ so $c_{2} \in C_{3}^{*}$. Now $X \cap C_{3}^{*}=\left\{d_{0}, c_{2}\right\}$. Since $C_{1}^{*} \cup d_{2}$ contains a circuit consisting of at least four elements, this circuit is $\left\{c_{1}, d_{1}, x, d_{2}\right\}$, by orthogonality. But now $C_{4}^{*}$ meets $\left\{c_{1}, d_{1}, x\right\}$ and 
$C_{0}-d_{2}=\left\{c_{1}, c_{2}, d_{0}\right\}$ and $C_{2}-e=\left\{c_{2}, x, d_{1}\right\}$, by orthogonality. As no element is in the intersection of these three sets, $C_{4}^{*} \subseteq X \cup e$; a contradiction.

We deduce that $C_{2} \subseteq X$. Recall that $c_{1} \notin C_{2}$. So let $C_{2}=\left\{c_{2}, x, d_{1}, e\right\}$ where $e \in\left\{d_{2}, d_{0}\right\}$. We will show that if $e=d_{0}$, then $X$ is a skew-whiff 3 -separator (see Figure 66). But first we consider the case where $e=d_{2}$.

Let $C_{2}=\left\{c_{2}, x, d_{1}, d_{2}\right\}$. Since $r(X)=4$ and $C_{1}^{*}$ is independent, $c_{2}$ is in a circuit that is contained in $C_{1}^{*} \cup c_{2}$. If this circuit is properly contained in $C_{1}^{*} \cup c_{2}$, then it consists of four elements, and hence it contains either $\left\{d_{1}, x\right\}$ and one of $c_{1}$ and $d_{0}$, or $\left\{c_{1}, d_{0}\right\}$ and one of $d_{1}$ and $x$. In any such case, due to the circuits $C_{0}$ and $C_{2}$, it follows that $r(X)=3$; a contradiction. So $C_{1}^{*} \cup c_{2}$ is a circuit. Similarly, we deduce that $C_{1}^{*} \cup d_{2}$ is a circuit. Now $X$ is in fact a twisted-cube-like 3-separator of $M^{*}$, but there is still the circuit $C_{1}$ containing $\left\{c_{1}, x, d_{2}\right\}$ to consider. If $C_{1} \subseteq X$, then $r(X)=3$; a contradiction. So let $C_{1}=\left\{c_{1}, x, d_{2}, g\right\}$, where $g \in E(M)-X$.

As $g \in \operatorname{cl}(X)$, and $X$ and $X \cup g$ are exactly 3-separating, we have $g \notin$ $\mathrm{cl}^{*}(X)$. Since $M \backslash d_{0} \backslash d_{1} / c_{1} / x$ has an $N$-minor, it follows that $\left\{g, d_{0}\right\}$ is $N$ deletable in $M \backslash d_{1}$ and $M$. So there is a 4-element cocircuit $C_{3}^{*}$ containing $\left\{g, d_{0}\right\}$ that is not contained in $X \cup g$. By orthogonality, $C_{3}^{*}$ meets $C_{0}-d_{0}=$ $\left\{c_{1}, c_{2}, d_{2}\right\}$ and $C_{1}-g=\left\{c_{1}, x, d_{2}\right\}$. As $C_{3}^{*} \nsubseteq X \cup g$, we deduce that $C_{3}^{*}$ meets $\left\{c_{1}, d_{2}\right\}$. But if $d_{2} \in C_{3}^{*}$, then $C_{3}^{*}$ intersects $C_{2}$ in two elements; a contradiction. So $C_{3}^{*}=\left\{g, d_{0}, c_{1}, h\right\}$ for some $h \in E(M)-(X \cup g)$.

Recall that $M \backslash d_{0} \backslash d_{1} \backslash g$ has an $N$-minor. As $\left\{c_{1}, x\right\}$ and $\left\{c_{1}, h\right\}$ are series pairs in this matroid, we see that $M / x / h$ has an $N$-minor. So the pair $\{x, h\}$ is contained in a 4-element circuit $C_{3}$ that, by orthogonality, meets $C_{1}^{*}-x$ and $C_{2}^{*}-x$. Since $h \in \mathrm{cl}^{*}(X \cup g)$, the set $X \cup\{g, h\}$ is 3-separating. Moreover, either $X \cup\{g, h\}$ is exactly 3-separating, for otherwise $|E(M)|=9$. So we may assume that $h \notin \operatorname{cl}(X \cup g)$; in particular, $C_{3} \nsubseteq X \cup\{g, h\}$. Since $d_{0}$ is the only element in the intersection of $C_{1}^{*}-x$ and $C_{2}^{*}-x$, we see that the $d_{0} \in C_{3}$. Let $C_{3}=\left\{x, h, d_{0}, q\right\}$ for $q \in E(M)-(X \cup\{g, h\})$.

Now, $q \in \operatorname{cl}(X \cup\{g, h\})$, so $X \cup\{g, h, q\}$ is 3 -separating. If this set is not exactly 3 -separating, then $|E(M)|=10$. So we may assume that $q \notin \mathrm{cl}^{*}(X \cup\{g, h\})$. Recall that $M \backslash d_{1} / x / h$ has an $N$-minor. It follows that $\left\{q, d_{1}\right\}$ is $N$-deletable, implying that this pair is contained in a 4-element cocircuit. By orthogonality, this cocircuit meets $C_{2}-d_{1}=\left\{x, c_{2}, d_{2}\right\}$. But if it meets $\left\{c_{2}, d_{2}\right\}$, then it intersects $C_{0}=\left\{c_{1}, d_{0}, c_{2}, d_{2}\right\}$ in at least two elements. Then $q$ is in a cocircuit contained in $X \cup q$, so $q \in \mathrm{cl}^{*}(X \cup\{g, h\})$; a contradiction. So the cocircuit contains $x$. Hence it meets $C_{1}-x=$ $\left\{c_{1}, d_{2}, g\right\}$. Again, we deduce the contradiction that $q \in \operatorname{cl}^{*}(X \cup\{g, h\})$.

Finally, we let $C_{2}=\left\{c_{2}, x, d_{1}, d_{0}\right\}$. Recall the 4-element circuit $C_{1}$ containing $\left\{c_{1}, x, d_{2}\right\}$. Let $C_{1}=\left\{c_{1}, x, d_{2}, g\right\}$. Suppose that $g \notin X$. Since $M \backslash d_{0} / c_{1} / x$ has an $N$-minor, $\left\{g, d_{0}\right\}$ is $N$-deletable. So $\left\{g, d_{0}\right\}$ is contained in a 4 -element cocircuit that, by orthogonality with $C_{0}, C_{1}$, and $C_{2}$, meets $\left\{c_{1}, c_{2}, d_{2}\right\},\left\{c_{1}, x, d_{2}\right\}$ and $\left\{c_{2}, x, d_{1}\right\}$. Since no element is contained in all 
three of these sets, $g \in \mathrm{cl}^{*}(X)-X$. But $X$ is 3-separating and $g \in \operatorname{cl}(X)$, so this is contradictory. Hence $g \in X$.

Recall that $c_{2} \notin C_{1}$, so $g \in\left\{d_{0}, d_{1}\right\}$. But if $g=d_{0}$, then $x \in$ $\operatorname{cl}\left(\left\{c_{1}, d_{0}, d_{2}\right\}\right)$, and, due to the circuits $C_{0}$ and $C_{2}$, it follows that $\left\{c_{1}, d_{0}, d_{2}\right\}$ spans $X$, and $r(X)<4$; a contradiction. Thus $g=d_{1}$. Now $X$ is a skewwhiff 3 -separator of $M$, labelled as in Figure 6 .

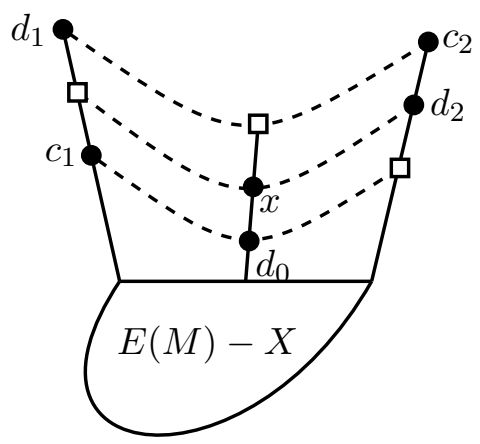

FiguRE 6. The labelling of the skew-whiff 3-separator of $M$ that arises in 4.4.3, where $\left\{d_{0}, d_{1}, d_{2}\right\} \subseteq D$ and $\left\{c_{1}, c_{2}\right\} \subseteq C$.

It remains to show that $E(M)-E(N) \subseteq X$. Towards a contradiction, suppose the $N$-labelling $(C, D)$ of $M$, with $\left\{c_{1}, c_{2}\right\} \subseteq C$ and $\left\{d_{0}, d_{1}, d_{2}\right\} \subseteq D$, also has either some element $c^{\prime} \in C-X$ or $d^{\prime} \in D-X$.

First, we show that if there is some such $c^{\prime}$, then any 4-element circuit containing $c^{\prime}$ and an element of $C \cap X$ is not contained in $X \cup c^{\prime}$. Let $c^{\prime}$ be such an element, and consider the 4-element circuit $C_{3}$ containing $\left\{c^{\prime}, c_{1}\right\}$. Suppose $C_{3} \subseteq X \cup c^{\prime}$. By orthogonality with $C_{0}^{*}, C_{1}^{*}$, and $C_{2}^{*}$, the circuit $C_{3}$ is $\left\{c^{\prime}, c_{1}, a, b\right\}$ for some $a \in\left\{x, d_{0}\right\}$ and $b \in\left\{c_{2}, d_{2}\right\}$. But if $a=x$, then, up to an $N$-label switch on $x$ and $b$, the pair $\left\{x, d_{1}\right\}$ is $N$-labelled for deletion, and $\left\{x, d_{1}\right\} \subseteq \operatorname{cl}^{*}\left(C_{0}\right)-C_{0}$, so (ii) holds by 4.4.1. So $a=d_{0}$, and, similarly, $b=d_{2}$. Now $C_{3}=\left\{c_{1}, c^{\prime}, d_{0}, d_{2}\right\}$, so $C_{0} \cup C_{3}$ is a 5 -element plane that intersects the cocircuit $C_{1}^{*}$ in two elements; a contradiction. So $c^{\prime}$ is not in a 4-element circuit contained in $X \cup c^{\prime}$, as claimed. Dually, if there exists some $d^{\prime} \in D-X$, then any 4-element cocircuit containing $d^{\prime}$ and an element in $D \cap X$ is not contained in $X \cup d^{\prime}$.

Next we show that if there is some $d^{\prime} \in D-X$, then there is also some $c^{\prime} \in C-X$. Suppose $d^{\prime} \in D-X$. Then $d^{\prime}$ is contained in a 4-element cocircuit with $d_{1}$, and an element $c^{\prime} \in E(M)-X$. By orthogonality, the final element in this cocircuit is $x$. Up to an $N$-label switch on $x$ and $c^{\prime}$, the element $c^{\prime}$ is $N$-labelled for contraction.

So we may assume there is an element $c^{\prime} \in C-X$. Since the 4-element circuit $C_{3}$ containing $\left\{c^{\prime}, c_{1}\right\}$ is not contained in $X \cup c^{\prime}$, it follows from orthogonality that $C_{3}=\left\{c^{\prime}, c_{1}, d_{1}, z_{1}\right\}$ for some $z_{1} \in E(M)-\left(X \cup c^{\prime}\right)$. Similarly, the 4-element circuits containing $\left\{c^{\prime}, c_{2}\right\}$ and $\left\{c^{\prime}, x\right\}$ are $C_{4}=$ $\left\{c^{\prime}, c_{2}, d_{2}, z_{2}\right\}$ and $C_{5}=\left\{c^{\prime}, x, d_{0}, z\right\}$ for some $z, z_{2} \in E(M)-\left(X \cup c^{\prime}\right)$. 
By an $N$-label switch on $d_{1}$ and $z_{1}$, we may assume $z_{1} \in D$. So there is a 4-element cocircuit $C_{3}^{*}$ containing $\left\{z_{1}, d_{0}\right\}$. As this cocircuit is not contained in $X \cup z_{1}$, it follows from orthogonality that $c_{2} \in C_{3}^{*}$. Now, by orthogonality with $C_{3}$, the final element is in $\left\{c_{1}, d_{1}, c^{\prime}\right\}$. Again using that $C_{3}^{*} \nsubseteq X \cup z_{1}$, we deduce that $C_{3}^{*}=\left\{z_{1}, d_{0}, c_{2}, c^{\prime}\right\}$. In particular, $r^{*}\left(X \cup\left\{c^{\prime}, z_{1}\right\}\right) \leq 5$. By symmetry, we deduce that $r^{*}\left(X \cup\left\{c^{\prime}, z_{2}\right\}\right) \leq 5$ and $r^{*}\left(X \cup\left\{c^{\prime}, z\right\}\right) \leq 5$.

Suppose that $z_{1}=z_{2}$. Then $\left\{z_{1}, c^{\prime}, c_{1}, c_{2}\right\}$ spans $X \cup\left\{c^{\prime}, z_{1}\right\}$, so $r(X \cup$ $\left.\left\{c^{\prime}, z_{1}\right\}\right)=4$. But $r^{*}\left(X \cup\left\{c^{\prime}, z_{1}\right\}\right) \leq 5$, so $\lambda\left(X \cup\left\{c^{\prime}, z_{1}\right\}\right) \leq 1$. It follows that $|E(M)|=9$; a contradiction. So $z_{1} \neq z_{2}$. By symmetry, $z, z_{1}$, and $z_{2}$ are pairwise distinct.

Now consider the cocircuit $C_{4}^{*}$ containing $\left\{z_{1}, d_{2}\right\}$. Using orthogonality and the fact that $C_{4}^{*} \nsubseteq X \cup z_{1}$, we have $c_{1} \in C_{4}^{*}$. Then, by orthogonality with $C_{4}$, and since $C_{4}^{*} \nsubseteq X \cup z_{1}$ again, the cocircuit $C_{4}^{*}$ meets $\left\{c^{\prime}, z_{2}\right\}$. But then $C_{4}^{*}$ intersects $C_{5}$ in at most one element, $c^{\prime}$, so $C_{4}^{*}=\left\{z_{1}, d_{2}, c_{1}, z_{2}\right\}$. Now $r^{*}(X \cup$ $\left.\left\{c^{\prime}, z_{1}, z_{2}\right\}\right) \leq 5$. As $r\left(X \cup\left\{c^{\prime}, z_{1}, z_{2}\right\}\right) \leq 5$, we have $\lambda\left(X \cup\left\{c^{\prime}, z_{1}, z_{2}\right\}\right) \leq 1$. It follows that $|E(M)|=10$; a contradiction. This completes the proof of 4.4 .3 .

4.4.4. Suppose $\left|C_{1}^{*} \cap C_{2}^{*}\right|=2$. If $C_{1}^{*} \triangle C_{2}^{*}$ is a circuit, then the lemma holds.

Subproof. Recall that $X=C_{1}^{*} \cup C_{2}^{*}$, and observe that $r^{*}(X)=4$, by 4.4 .2 and since $\left|C_{1}^{*} \cap C_{2}^{*}\right|=2$. Suppose $C_{0}=C_{1}^{*} \triangle C_{2}^{*}$ is a circuit.

We claim that $d_{1} \notin C_{2}^{*}$. Towards a contradiction, suppose $d_{1} \in C_{2}^{*}$. Recall that $d_{2} \notin C_{1}^{*}$. So, without loss of generality, let $C_{1}^{*}=\left\{d_{0}, d_{1}, c_{1}, c^{\prime}\right\}$ and $C_{2}^{*}=\left\{d_{0}, d_{1}, d_{2}, c_{2}\right\}$ where $c_{1}$ and $c_{2}$ are $N$-labelled for contraction. Since $C_{0}$ contains $\left\{c_{1}, c_{2}\right\}$, it is a coindependent circuit, so it cospans $X$. Hence $\left\{d_{0}, d_{1}\right\} \subseteq \mathrm{cl}^{*}\left(C_{0}\right)-C_{0}$, and it follows that (ii) holds by 4.4.1, a contradiction. So $d_{1} \notin C_{2}^{*}$, as claimed.

Now let $C_{1}^{*}=\left\{d_{1}, c_{1}, d_{0}, x\right\}$ and $C_{2}^{*}=\left\{d_{2}, c_{2}, d_{0}, x\right\}$ for distinct $c_{1}, c_{2}, x \in$ $E(M)-\left\{d_{0}, d_{1}, d_{2}\right\}$. Since $M \backslash d_{0} \backslash d_{1} \backslash d_{2}$ has an $N$-minor, and $\left\{x, c_{1}, c_{2}\right\}$ is contained in a series class in this matroid, the pairs $\left\{x, c_{1}\right\}$ and $\left\{x, c_{2}\right\}$ are $N$-contractible. Let $C_{1}$ and $C_{2}$ be the 4-element circuits containing $\left\{x, c_{1}\right\}$ and $\left\{x, c_{2}\right\}$ respectively. Observe that $\left|C_{1} \cap X\right| \geq 3$ and $\left|C_{2} \cap X\right| \geq 3$, by orthogonality.

If $X$ is 3-separating, then (i) holds by 4.4.3. So $\lambda(X) \geq 3$. Since $r^{*}(X)=4$ and $X$ contains a circuit, $r(X)=5$. Hence, $C_{1} \nsubseteq X$ and $C_{2} \nsubseteq X$, so $\left|C_{1} \cap X\right|=\left|C_{2} \cap X\right|=3$. So let $C_{1}=\left\{c_{1}, x, x_{1}, g_{1}\right\}$ and $C_{2}=\left\{c_{2}, x, x_{2}, g_{2}\right\}$ where $x_{1}, x_{2} \in X$ and $g_{1}, g_{2} \in E(M)-X$. If $x_{1}=c_{2}$, then $\left\{d_{0}, x\right\} \subseteq D$ up to an $N$-label switch, and $\left\{d_{0}, x\right\} \subseteq \mathrm{cl}^{*}\left(C_{0}\right)-C_{0}$ since $C_{0}$ is coindependent, in which case (ii) holds by 4.4.1. So we may assume that $c_{2} \notin C_{1}$ and, by symmetry, $c_{1} \notin C_{2}$. Hence, by orthogonality, $x_{1} \in\left\{d_{0}, d_{2}\right\}$ and $x_{2} \in$ $\left\{d_{0}, d_{1}\right\}$. If $g_{1} \in \mathrm{cl}^{*}(X)$, then the coindependent circuit $C_{1}$ cospans $X \cup g_{1}$. In this case, the pair $\left\{d_{0}, d_{1}, d_{2}\right\}-x_{1}$ is $N$-labelled for deletion, and contained in $\mathrm{cl}^{*}\left(C_{1}\right)-C_{1}$. Thus (ii) holds by 4.4.1. So we may assume that $g_{1} \notin \mathrm{cl}^{*}(X)$. Similarly, $g_{2} \notin \mathrm{cl}^{*}(X)$. 
Suppose that $x_{1}=d_{2}$, so $C_{1}=\left\{c_{1}, x, d_{2}, g_{1}\right\}$. Let $C_{0}^{*}$ be the 4-element cocircuit containing $\left\{d_{1}, d_{2}\right\}$. By orthogonality, $C_{0}^{*}$ meets $\left\{c_{1}, x, g_{1}\right\}$.

We claim that $g_{1} \notin C_{0}^{*}$. Towards a contradiction, suppose $g_{1} \in C_{0}^{*}$. Then, as $g_{1} \notin \mathrm{cl}^{*}(X)$, we have $C_{0}^{*}=\left\{d_{1}, d_{2}, g_{1}, f\right\}$ for some $f \in E(M)-\left(X \cup g_{1}\right)$. As $M \backslash d_{0} \backslash d_{1} / c_{1} / x$ has an $N$-minor, and $\left\{d_{2}, g_{1}\right\}$ is a parallel pair in this matroid, $M \backslash d_{0} \backslash d_{1} \backslash g_{1} / c_{1}$ has an $N$-minor. Now $\left\{d_{2}, f\right\}$ is a series pair in this matroid, so $M \backslash d_{0} / c_{1} / d_{2}$ has an $N$-minor. Finally, $\left\{x, g_{1}\right\}$ is a parallel pair in this matroid, so $\left\{d_{0}, x\right\}$ is $N$-deletable. But $\left\{d_{0}, x\right\} \subseteq \mathrm{cl}^{*}\left(C_{0}\right)-C_{0}$, so (ii) holds in this case, by 4.4.1. So we may assume that $g_{1} \notin C_{0}^{*}$.

Recall that $x_{2} \in\left\{d_{0}, d_{1}\right\}$; we now distinguish two subcases, depending on whether $x_{2}=d_{0}$ or $x_{2}=d_{1}$. We first handle the case that $x_{2}=d_{1}$, so $C_{2}=\left\{c_{2}, x, d_{1}, g_{2}\right\}$. Then, by orthogonality, $C_{0}^{*}$ meets $\left\{c_{2}, x, g_{2}\right\}$. By the argument in the previous paragraph, $g_{2} \notin C_{0}^{*}$. Hence $C_{0}^{*}$ meets $\left\{c_{1}, x\right\}$ and $\left\{c_{2}, x\right\}$. Since $r^{*}(X)=4$ and $C_{0}$ is coindependent, $C_{0}^{*}=\left\{d_{1}, d_{2}, x, f\right\}$ for some $f \in E(M)-\left(X \cup\left\{g_{1}, g_{2}\right\}\right)$. As any pair in $\mathrm{cl}^{*}(X)-\left\{d_{0}, d_{1}, d_{2}\right\}$ is $N$-contractible, and $\{f, x\}$ is such a pair, $\{f, x\}$ is contained in a 4-element circuit $C_{3}$. By orthogonality, this circuit meets $C_{1}^{*}-x$ and $C_{2}^{*}-x$. Thus, if $C_{3} \nsubseteq \subseteq X \cup f$, then $d_{0} \in C_{3}$. However, $X-x$ also contains a cocircuit, by cocircuit elimination on $C_{1}^{*}$ and $C_{2}^{*}$, and this cocircuit contains $d_{0}$, since $C_{0}$ is coindependent. So $C_{3} \subseteq X \cup f$. Now $C_{3}$ cospans $X \cup f$, so if there is a pair of $N$-deletable elements in $(X \cup f)-C_{3}$, then (ii) holds by 4.4.1. So $C_{3}=\left\{x, f, d^{\prime}, d^{\prime \prime}\right\}$ for some distinct $d^{\prime}, d^{\prime \prime} \in\left\{d_{0}, d_{1}, d_{2}\right\}$. Moreover, as $C_{0}^{*}$ is independent, $C_{3} \neq C_{0}^{*}$. So, without loss of generality, $C_{3}=\left\{x, f, d_{0}, d_{1}\right\}$.

As $\left\{c_{1}, c_{2}, x, f\right\}$ is contained in a series class in $M \backslash d_{0} \backslash d_{1} \backslash d_{2}$, the matroid $M / c_{1} / c_{2} / x$ has an $N$-minor. As $\left\{g_{1}, d_{2}\right\}$ and $\left\{d_{1}, g_{2}\right\}$ are parallel pairs in this matroid, it follows that $M \backslash g_{1} \backslash d_{1}$ has an $N$-minor. So the pair $\left\{g_{1}, d_{1}\right\}$ is contained in a 4-element cocircuit $C_{3}^{*}$. By orthogonality with $C_{0}$ and $C_{1}$, this cocircuit meets $\left\{c_{1}, c_{2}, d_{2}\right\}$ and $\left\{c_{1}, x, d_{2}\right\}$. Thus, if $C_{3}^{*}$ does not meet $\left\{c_{1}, d_{2}\right\}$, then $C_{3}^{*} \subseteq X \cup g_{1}$, implying $g_{1} \in \operatorname{cl}^{*}(X)$; a contradiction. So $C_{3}^{*}$ meets $\left\{c_{1}, d_{2}\right\}$. But, by orthogonality with $C_{2}$ and $C_{3}$, the cocircuit $C_{3}^{*}$ also meets $\left\{c_{2}, x, g_{2}\right\}$ and $\left\{x, f, d_{0}\right\}$. Thus $x \in C_{3}^{*}$, and again we arrive at the contradiction that $g_{1} \in \mathrm{cl}^{*}(X)$. This completes the case where $x_{1}=d_{2}$ and $x_{2}=d_{1}$.

We now consider the subcase where $x_{2}=d_{0}$, so $C_{2}=\left\{c_{2}, x, d_{0}, g_{2}\right\}$. Recall that $C_{1}=\left\{c_{1}, x, d_{2}, g_{1}\right\}$, and the 4-element cocircuit $C_{0}^{*}$ that contains $\left\{d_{1}, d_{2}\right\}$ meets $\left\{c_{1}, x\right\}$. As $r^{*}(X)=4$ and $C_{0}^{*} \neq C_{0}$ (as $C_{0}$ is coindependent), $C_{0}^{*} \nsubseteq X$. If $x \in C_{0}^{*}$, then $C_{0}^{*}$ also meets $C_{2}-x=\left\{c_{2}, d_{0}, g_{2}\right\}$, by orthogonality, so $C_{0}^{*}=\left\{d_{1}, d_{2}, x, g_{2}\right\}$, implying $g_{2} \in \mathrm{cl}^{*}(X)$; a contradiction. So $C_{0}^{*}=\left\{d_{1}, d_{2}, c_{1}, f\right\}$ where $f \in E(M)-X$.

Since $M \backslash d_{0} \backslash d_{1} \backslash d_{2} / x$ has an $N$-minor, and $\left\{c_{1}, f\right\}$ is a series pair in this matroid, $\{f, x\}$ is $N$-contractible in $M \backslash d_{0}$ and $M$. So $\{f, x\}$ is contained in a 4 -element circuit $C_{4}$. As $C_{4}$ cospans $X \cup f$, if $\left|\left\{d_{0}, d_{1}, d_{2}\right\}-C_{4}\right| \geq 2$, then there is a pair of $N$-deletable elements in $\mathrm{cl}^{*}\left(C_{4}\right)-C_{4}$, so (ii) holds by 4.4.1. So $C_{4}=\left\{f, x, d^{\prime}, d^{\prime \prime}\right\}$ for some distinct $d^{\prime}, d^{\prime \prime} \in\left\{d_{0}, d_{1}, d_{2}\right\}$. We may assume that $d^{\prime} \in\left\{d_{1}, d_{2}\right\}$. Observe that $\left\{g_{2}, d^{\prime}\right\}$ is $N$-deletable for any 
such $d^{\prime}$. Thus there is a 4-element cocircuit $C_{4}^{*}$ containing $\left\{g_{2}, d^{\prime}\right\}$. This cocircuit meets both $C_{2}-g_{2}=\left\{c_{2}, x, d_{0}\right\}$ and $C_{0}-d^{\prime}$, by orthogonality. Since $g_{2} \notin \mathrm{cl}^{*}(X)$, we have $c_{2} \in C_{4}^{*}$. By orthogonality with $C_{4}$, we see that $C_{4}^{*}$ also meets $\left\{f, x, d^{\prime \prime}\right\}$. But then $g_{2} \in \operatorname{cl}^{*}(X \cup f)=\operatorname{cl}^{*}(X)$; a contradiction. This completes the case where $x_{1}=d_{2}$.

Now we may assume that $x_{1} \neq d_{2}$, and, by symmetry, $x_{2} \neq d_{1}$. Hence $x_{1}=x_{2}=d_{0}$, so $C_{1}=\left\{c_{1}, x, d_{0}, g_{1}\right\}$ and $C_{2}=\left\{c_{2}, x, d_{0}, g_{2}\right\}$. If $g_{1}=g_{2}$, then $\left\{c_{1}, c_{2}, x, d_{0}\right\}$ contains a circuit, by circuit elimination on $C_{1}$ and $C_{2}$, but then $r(X) \leq 4$; a contradiction. So $g_{1} \neq g_{2}$. Since $M \backslash d_{1} \backslash d_{2} / c_{2} / x$ has an $N$-minor, and $\left\{d_{0}, g_{2}\right\}$ is a parallel pair in this matroid, we see that any pair contained in $\left\{g_{2}, d_{1}, d_{2}\right\}$ is $N$-deletable in $M / x$ and $M$. In particular, $\left\{g_{2}, d_{1}\right\}$ is contained in a 4 -element cocircuit $C_{3}^{*}$. By orthogonality, this cocircuit meets $C_{2}-g_{2}=\left\{c_{2}, x, d_{0}\right\}$ and $C_{0}-d_{1}=\left\{c_{1}, c_{2}, d_{2}\right\}$. Since $g_{2} \notin \mathrm{cl}^{*}(X)$, we deduce that $c_{2} \in C_{3}^{*}$. So let $C_{3}^{*}=\left\{g_{2}, d_{1}, c_{2}, h_{1}\right\}$, where $h_{1} \in E(M)-\left(X \cup g_{2}\right)$. Also, we let $C_{4}^{*}$ be the 4-element cocircuit containing $\left\{g_{2}, d_{2}\right\}$, and observe, similarly, that $c_{2} \in C_{4}^{*}$. So $C_{4}^{*}=\left\{g_{2}, d_{2}, c_{2}, h_{2}\right\}$ for some $h_{2} \in E(M)-\left(X \cup g_{2}\right)$.

Recall that $M \backslash d_{1} \backslash g_{2} / x$ has an $N$-minor; it follows that $\left\{x, h_{1}\right\}$ is $N$ contractible. So let $C_{3}$ be the 4-element circuit containing $\left\{x, h_{1}\right\}$. By orthogonality, $C_{3}$ meets $C_{1}^{*}-x=\left\{c_{1}, d_{1}, d_{0}\right\}$ and $C_{2}^{*}-x=\left\{d_{0}, c_{2}, d_{2}\right\}$, as well as $C_{3}^{*}-h_{1}=\left\{g_{2}, d_{1}, c_{2}\right\}$.

Suppose that $g_{2} \in C_{3}$. Then $C_{3}=\left\{x, h_{1}, g_{2}, d_{0}\right\}$. But $X-x$ contains a cocircuit, by cocircuit elimination of $C_{1}^{*}$ and $C_{2}^{*}$, and this cocircuit must contain $d_{0}$, as $C_{0}$ is coindependent. So $X-x$ is a cocircuit that intersects $C_{3}$ in a single element; a contradiction. So $C_{3}$ meets $\left\{d_{1}, c_{2}\right\}$.

We claim that $c_{2} \notin C_{3}$. Suppose $c_{2} \in C_{3}$. Then $C_{3}$ also meets $\left\{c_{1}, d_{0}, d_{1}\right\}$. Let $C_{3}=\left\{x, h_{1}, c_{2}, c_{1}\right\}$. Then, as $M \backslash d_{0} / c_{1} / c_{2}$ has an $N$-minor, $M \backslash d_{0} \backslash x$ has an $N$-minor. But $\left\{d_{0}, x\right\} \subseteq \mathrm{cl}^{*}\left(C_{0}\right)-C_{0}$, so (ii) holds by 4.4.1. Now let $C_{3}=\left\{x, h_{1}, c_{2}, d_{0}\right\}$. Then, by circuit elimination with $C_{2}$, there is a circuit contained in $\left\{x, h_{1}, c_{2}, g_{2}\right\}$. But this set intersects $C_{1}^{*}$ in a single element $x$, so $\left\{h_{1}, c_{2}, g_{2}\right\}$ is a triangle; a contradiction. So if $c_{2} \in C_{3}$, then $C_{3}=\left\{x, h_{1}, c_{2}, d_{1}\right\}$.

Let $C_{3}=\left\{x, h_{1}, c_{2}, d_{1}\right\}$. Then, as $M \backslash d_{2} / x / c_{2}$ has an $N$-minor, where $\left\{d_{0}, g_{2}\right\}$ and $\left\{h_{1}, d_{1}\right\}$ are parallel pairs in this matroid, $M \backslash d_{2} \backslash g_{2} \backslash h_{1} / x$ has an $N$-minor. Now, $\left\{d_{1}, c_{2}\right\}$ is a series pair in this matroid, due to $C_{3}^{*}$, so $M \backslash d_{2} \backslash g_{2} / x / d_{1}$ has an $N$-minor. As $\left\{c_{2}, h_{2}\right\}$ is a series pair in this matroid, due to $C_{4}^{*}$, the matroid $M / x / d_{1} / c_{2}$ has an $N$-minor. In turn, $\left\{d_{0}, g_{2}\right\}$ is a parallel pair in this matroid, due to $C_{2}$, so $M \backslash d_{0} / d_{1} / c_{2}$ has an $N$-minor. Finally, $\left\{h_{1}, x\right\}$ is a parallel pair in this matroid, due to $C_{3}$, so $M \backslash d_{0} \backslash x$ has an $N$-minor. But $\left\{d_{0}, x\right\} \subseteq \operatorname{cl}^{*}\left(C_{0}\right)-C_{0}$. So (ii) holds, by 4.4.1, This proves the claim that $c_{2} \notin C_{3}$. It now follows that $C_{3}=\left\{x, h_{1}, d_{1}, d^{\prime}\right\}$ for $d^{\prime} \in\left\{d_{0}, d_{2}\right\}$.

Suppose that $h_{1}=h_{2}$. Then, by cocircuit elimination on $C_{3}^{*}$ and $C_{4}^{*}$, there is a cocircuit $\left\{g_{2}, d_{1}, d_{2}, c_{2}\right\}$. But then $g_{2} \in \mathrm{cl}^{*}(X)$; a contradiction. So $h_{1} \neq h_{2}$. 
Now, if $d^{\prime}=d_{2}$, so $C_{3}=\left\{x, h_{1}, d_{1}, d_{2}\right\}$, then, as $h_{1} \notin\left\{h_{2}, g_{2}\right\}$, the circuit $C_{3}$ intersects $C_{4}^{*}$ in a single element $d_{2}$; a contradiction. So $d^{\prime}=d_{0}$; that is, $C_{3}=\left\{x, h_{1}, d_{1}, d_{0}\right\}$. Similarly, by symmetry we obtain $C_{4}=\left\{x, h_{2}, d_{2}, d_{0}\right\}$.

Consider the 4-element cocircuit $C_{0}^{*}$ containing $\left\{d_{1}, d_{2}\right\}$. By orthogonality with $C_{3}$, the cocircuit $C_{0}^{*}$ meets $\left\{x, h_{1}, d_{0}\right\}$. But if $C_{0}^{*}$ meets $\left\{x, d_{0}\right\}$, then, by orthogonality with $C_{1}$ and $C_{2}$, and since $g_{1} \neq g_{2}$, we have $C_{0}^{*}=\left\{d_{1}, d_{2}, x, d_{0}\right\}$. Then $r^{*}(X)=3$, contradicting 4.4.2. So $h_{1} \in C_{0}^{*}$. Similarly, using $C_{4}$ in place of $C_{3}$, we see that $C_{0}^{*}=\left\{d_{1}, d_{2}, h_{1}, h_{2}\right\}$.

Using a similar approach taken to reveal the cocircuits $C_{3}^{*}$ and $C_{4}^{*}$, we can observe that the pairs $\left\{g_{1}, d_{1}\right\}$ and $\left\{g_{1}, d_{2}\right\}$ are $N$-deletable, so are contained in 4-element cocircuits $C_{5}^{*}$ and $C_{6}^{*}$ respectively, each containing $c_{1}$. These cocircuits meet $C_{3}$ and $C_{4}$, respectively, but neither cocircuit meets $C_{2}$. It follows, by orthogonality, that $C_{5}^{*}=\left\{g_{1}, d_{1}, c_{1}, h_{1}\right\}$ and $C_{6}^{*}=\left\{g_{1}, d_{2}, c_{1}, h_{2}\right\}$.

As $M \backslash d_{1} \backslash d_{2} / c_{1}$ has an $N$-minor, and $\left\{h_{1}, h_{2}\right\}$ is a series pair in this matroid due to the cocircuit $C_{0}^{*}$, the pair $\left\{c_{1}, h_{1}\right\}$ is $N$-contractible. Consider the 4-element circuit $C_{5}$ containing $\left\{c_{1}, h_{1}\right\}$. By orthogonality with $C_{1}^{*}, C_{3}^{*}$, and $C_{6}^{*}$, it meets $\left\{d_{1}, x, d_{0}\right\},\left\{g_{2}, d_{1}, c_{2}\right\}$, and $\left\{g_{1}, d_{2}, h_{2}\right\}$. Since the last two sets are disjoint, $C_{5}$ does not meet $\left\{x, d_{0}\right\}$, so $d_{1} \in C_{5}$. The final element in $C_{5}$ is in $\left\{g_{1}, d_{2}, h_{2}\right\}$. Now $C_{5}$ intersects $C_{2}^{*}$ and $C_{4}^{*}$ in at most one element. By orthogonality, $C_{5}$ and $C_{2}^{*}$ are disjoint, so $d_{2} \notin C_{5}$; similarly, $C_{5} \cap C_{4}^{*}=\emptyset$, so $h_{2} \notin C_{5}$. Now $C_{5}=\left\{c_{1}, h_{1}, d_{1}, g_{1}\right\}=C_{5}^{*}$ is a quad containing the $N$ deletable pair $\left\{d_{1}, g_{1}\right\}$; a contradiction.

4.4.5. Suppose $C_{1}^{*}=\left\{d_{0}, x, c_{1}, d_{1}\right\}$ and $C_{2}^{*}=\left\{d_{0}, x, c_{2}, d_{2}\right\}$. If there is a 4-element circuit $C_{0}$ such that $\left\{c_{1}, c_{2}\right\} \subseteq C_{0} \subseteq C_{1}^{*} \cup C_{2}^{*}$, then the lemma holds.

Subproof. Let $X=C_{1}^{*} \cup C_{2}^{*}$. If $X$ is 3-separating, then (i) holds by 4.4.3, So we may assume that $X$ is not 3 -separating. Let $C_{0}$ be a 4 -element circuit containing $\left\{c_{1}, c_{2}\right\}$ and contained in $X$. As $r^{*}(X)=4$, by 4.4.2, it now follows that $r(X)=5$. If $x \in C_{0}$, then $X-C_{0}$ is a pair that is $N$-deletable. Since $C_{0}$ is coindependent, and $r^{*}(X)=4$, (ii) holds by 4.4.1 in this case. So we may assume that $x \notin C_{0}$. Now either $C_{0}=C_{1}^{*} \triangle C_{2}^{*}$ or, up to symmetry, $C_{0}=\left\{c_{1}, c_{2}, d_{0}, d_{2}\right\}$. But in the former case, the lemma holds by 4.4.4. So let $C_{0}=\left\{c_{1}, c_{2}, d_{0}, d_{2}\right\}$.

Let $C_{0}^{*}$ be the 4-element cocircuit containing $\left\{d_{1}, d_{2}\right\}$. By orthogonality, $C_{0}^{*}$ meets $C_{0}-d_{2}$. So let $C_{0}^{*}=\left\{d_{1}, d_{2}, y, f\right\}$, where $y \in\left\{d_{0}, c_{1}, c_{2}\right\}$ and $f \in$ $E(M)-\left\{d_{1}, d_{2}, y\right\}$. If $C_{0}^{*} \subseteq X$, then, as $r^{*}(X)=4$, we have $C_{0}^{*}=C_{1}^{*} \triangle C_{2}^{*}$. That is, either $\{y, f\}=\left\{c_{1}, c_{2}\right\}$ or $f \in E(M)-X$.

Let $C_{1}$ and $C_{2}$ be the 4-element circuits containing $\left\{c_{1}, x\right\}$ and $\left\{c_{2}, x\right\}$ respectively, and observe that $\left|C_{1} \cap X\right| \geq 3$ and $\left|C_{2} \cap X\right| \geq 3$, by orthogonality. Recall that $r(X)=5$. Hence, $C_{1} \nsubseteq X$ and $C_{2} \nsubseteq X$, so $\left|C_{1} \cap X\right|=\left|C_{2} \cap X\right|=3$. So let $C_{1}=\left\{c_{1}, x, x_{1}, g_{1}\right\}$ and $C_{2}=\left\{c_{2}, x, x_{2}, g_{2}\right\}$ where $x_{1}, x_{2} \in X$ and $g_{1}, g_{2} \in E(M)-X$. If $x_{1}=c_{2}$, then $\left\{d_{1}, x\right\}$ is $N$ deletable, and $\left\{d_{1}, x\right\} \subseteq \mathrm{cl}^{*}\left(C_{0}\right)-C_{0}$, so (ii) holds by 4.4.1. So we may assume that $c_{2} \notin C_{1}$ and, by symmetry, $c_{1} \notin C_{2}$. By orthogonality, we now 
have $x_{1} \in\left\{d_{0}, d_{2}\right\}$ and $x_{2} \in\left\{d_{0}, d_{1}\right\}$. If $g_{1} \in \mathrm{cl}^{*}(X)$, then the coindependent circuit $C_{1}$ cospans $X \cup g_{1}$. In this case, $\left\{d_{0}, d_{1}, d_{2}\right\}-x_{1}$ is a pair of $N$-deletable elements contained in $\mathrm{cl}^{*}\left(C_{1}\right)-C_{1}$. Thus (ii) holds by 4.4.1. So we may assume that $g_{1} \notin \mathrm{cl}^{*}(X)$. Similarly, $g_{2} \notin \mathrm{cl}^{*}(X)$.

Suppose $x_{1}=d_{0}$. If $C_{0}^{*}$ meets $C_{1}=\left\{c_{1}, x, d_{0}, g_{1}\right\}$, then it does so in at least two elements, by orthogonality. So in this case $C_{0}^{*}=\left\{d_{1}, d_{2}, y, g_{1}\right\}$ where $y \in\left\{d_{0}, c_{1}\right\}$. But then $g_{1} \in \operatorname{cl}^{*}(X)$; a contradiction. So we may assume that $C_{0}^{*}$ and $C_{1}$ are disjoint. Then $C_{0}^{*}=\left\{d_{1}, d_{2}, c_{2}, f\right\}$ for $f \in$ $E(M)-\left(X \cup g_{1}\right)$. Note also that $f \neq g_{2}$, since $f \in \mathrm{cl}^{*}(X)$ but $g_{2} \notin$ $\mathrm{cl}^{*}(X)$. Now $C_{2}$ and $C_{0}^{*}$ both contain the element $c_{2}$; hence we deduce, by orthogonality, that $x_{2}=d_{1}$. So $C_{2}=\left\{c_{2}, x, d_{1}, g_{2}\right\}$.

By cocircuit elimination, $X-x$ contains a cocircuit. By orthogonality with $C_{1}$, this cocircuit is not $C_{1}^{*} \triangle C_{2}^{*}$. So the cocircuit contains $d_{0}$. Since $r^{*}(X)=4$, by 4.4.2, it follows that $X-x$ is a cocircuit.

Since $M \backslash d_{1} \backslash d_{2} / x$ has an $N$-minor, and $\{f, y\}$ is a series pair in this matroid, $\{f, x\}$ is $N$-contractible. Let $C_{3}$ be the circuit containing $\{f, x\}$. By orthogonality, $C_{3}$ meets $C_{1}^{*}-x$ and thus, as $X-x$ is a cocircuit, it intersects $X-x$ in two elements. Now $C_{3}$ is coindependent, so it cospans the corank-4 set $X \cup f$. Thus if $X-C_{3}$ contains a pair of $N$-deletable elements, then (ii) holds by 4.4.1. So $\left|C_{3} \cap\left\{d_{0}, d_{1}, d_{2}\right\}\right|=2$.

As $M \backslash d_{0} \backslash d_{2} / c_{2} / x$ has an $N$-minor and $\left\{d_{1}, g_{2}\right\}$ is a parallel pair in this matroid, $\left\{g_{2}, d_{0}\right\}$ is $N$-deletable. So $\left\{g_{2}, d_{0}\right\}$ is contained in a 4-element cocircuit $C_{3}^{*}$. By orthogonality with $C_{0}, C_{1}$, and $C_{2}$, the cocircuit $C_{3}^{*}$ meets $\left\{c_{1}, c_{2}, d_{2}\right\},\left\{c_{1}, x, g_{1}\right\}$, and $\left\{c_{2}, x, d_{1}\right\}$. If $C_{3}^{*}$ meets $\left\{c_{1}, x\right\}$, then $C_{3}^{*} \subseteq X \cup$ $g_{2}$, contradicting that $g_{2} \notin \mathrm{cl}^{*}(X)$. So $g_{1} \in C_{3}^{*}$. It follows that $C_{3}^{*}=$ $\left\{g_{1}, g_{2}, d_{0}, c_{2}\right\}$. By orthogonality, we now deduce that $C_{3}=\left\{f, x, d_{1}, d_{2}\right\}$.

We claim that $\{f, x\}$ is $N$-deletable. First, observe that as $\left\{c_{1}, c_{2}, x, f\right\}$ is contained in a series class in $M \backslash d_{0} \backslash d_{1} \backslash d_{2}$, the matroid $M \backslash d_{0} \backslash d_{1} \backslash d_{2} / c_{1} / c_{2} / x$ has an $N$-minor. Due to the circuits $C_{1}$ and $C_{2}$, it follows that $M \backslash g_{1} \backslash g_{2} \backslash d_{2} / c_{1}$ has an $N$-minor. Now $\left\{d_{0}, c_{2}\right\}$ is a series pair in this matroid due to $C_{3}^{*}$, so $M \backslash g_{2} / c_{1} / d_{0}$ has an $N$-minor. As $\left\{c_{2}, d_{2}\right\}$ and $\left\{x, g_{1}\right\}$ are parallel pairs in this matroid, due to $C_{0}$ and $C_{1}$ respectively, the matroid $M \backslash g_{2} \backslash c_{2} \backslash x / c_{1}$ has an $N$-minor. As $\left\{d_{0}, d_{2}\right\}$ is a series pair in this matroid due to $C_{2}^{*}$, the matroid $M \backslash g_{2} \backslash x / c_{1} / d_{2}$ has an $N$-minor. Now $\left\{d_{0}, c_{2}\right\}$ is a parallel pair in this matroid due to $C_{0}$, so $M \backslash g_{2} \backslash x \backslash d_{0} / d_{2}$ has an $N$-minor. As $\left\{c_{1}, d_{1}\right\}$ and $\left\{g_{1}, c_{2}\right\}$ are series pairs in this matroid, due to $C_{1}^{*}$ and $C_{3}^{*}$ respectively, the matroid $M / c_{2} / d_{1} / d_{2}$ has an $N$-minor. Finally, $\{x, f\}$ and $\left\{x, g_{2}\right\}$ are parallel pairs in this matroid due to $C_{3}$ and $C_{2}$, so $M \backslash f \backslash x$ has an $N$-minor, as required.

Since $C_{0}$ cospans $X \cup f$, the $N$-deletable pair $\{f, x\}$ is in $\operatorname{cl}^{*}\left(C_{0}\right)-C_{0}$, implying that (ii) holds by 4.4.1.

Now we may assume that $x_{1}=d_{2}$ and, by symmetry, $x_{2}=d_{1}$, so that $C_{1}=\left\{c_{1}, x, d_{2}, g_{1}\right\}$ and $C_{2}=\left\{c_{2}, x, d_{1}, g_{2}\right\}$. If $g_{1}=g_{2}$, then $\left\{c_{1}, c_{2}, x, d_{1}, d_{2}\right\}$ contains a circuit, by circuit elimination, so $r\left(X-d_{0}\right) \leq 4$. But $d_{0} \in$ 
$\operatorname{cl}\left(X-d_{0}\right)$, due to the circuit $C_{0}$, so this is contradictory. So $g_{1} \neq g_{2}$. Now $C_{0}^{*}$ meets $C_{1}$ and $C_{2}$, but $x \notin C_{0}^{*}$ and $C_{0}^{*}$ contains at most one of $g_{1}$ and $g_{2}$. It follows, by orthogonality, that $C_{0}^{*}$ meets $\left\{c_{1}, c_{2}\right\}$; by symmetry, we may assume that $c_{1} \in C_{0}^{*}$. Then $C_{0}^{*}=\left\{d_{1}, d_{2}, c_{1}, f\right\}$ for some $f \in\left\{c_{2}, x, g_{2}\right\}$. But $f \neq x$, otherwise $r^{*}(X)=3$. If $f=g_{2}$, then $g_{2} \in \mathrm{cl}^{*}(X)$; a contradiction. So $f=c_{2}$ and $C_{0}^{*}=C_{1}^{*} \triangle C_{2}^{*}$.

As $M \backslash d_{0} \backslash d_{2} / c_{2} / x$ has an $N$-minor, $\left\{d_{0}, g_{2}, d_{2}\right\}$ is $N$-deletable. Let $C_{3}^{*}$ be the 4 -element cocircuit containing $\left\{g_{2}, d_{2}\right\}$. Recall that $g_{2} \notin \mathrm{cl}^{*}(X)$, so $C_{3}^{*} \nsubseteq X \cup g_{2}$. As $C_{3}^{*}$ meets $C_{0}-d_{2}=\left\{c_{1}, c_{2}, d_{0}\right\}$ and $C_{2}-g_{2}=\left\{c_{2}, x, d_{1}\right\}$, by orthogonality, it follows that $c_{2} \in C_{3}^{*}$. But $C_{3}^{*}$ also meets $C_{1}-d_{2}=$ $\left\{c_{1}, x, g_{1}\right\}$, so $g_{1} \in C_{3}^{*}$. That is, $C_{3}^{*}=\left\{g_{2}, d_{2}, c_{2}, g_{1}\right\}$.

Now $M \backslash d_{0} \backslash d_{2} \backslash g_{2}$ has an $N$-minor, and $\left\{x, c_{2}\right\}$ and $\left\{c_{2}, g_{1}\right\}$ are series pairs in this matroid. So $M \backslash g_{2} / x / g_{1}$ has an $N$-minor, where $\left\{c_{1}, d_{2}\right\}$ is a parallel pair in this matroid due to the circuit $C_{1}$, implying $\left\{g_{2}, c_{1}\right\}$ is $N$-deletable. Consider the cocircuit $C_{4}^{*}$ containing $\left\{g_{2}, c_{1}\right\}$. By orthogonality, $C_{4}^{*}$ meets $C_{0}-c_{1}=\left\{d_{0}, d_{2}, c_{2}\right\}$ and $C_{2}-g_{2}=\left\{d_{1}, x, c_{2}\right\}$. As $g_{2} \notin \mathrm{cl}^{*}(X)$, we have $c_{2} \in C_{4}^{*}$. By orthogonality with $C_{1}$, and again using that $g_{2} \notin \mathrm{cl}^{*}(X)$, we have $C_{4}^{*}=\left\{g_{1}, g_{2}, c_{1}, c_{2}\right\}$. Now $r^{*}\left(C_{3}^{*} \cup C_{4}^{*}\right)=3$, and $d_{1} \in \operatorname{cl}^{*}\left(C_{3}^{*} \cup C_{4}^{*}\right)$ due to the cocircuit $C_{0}^{*}$. It follows that $r^{*}\left(X \cup\left\{g_{1}, g_{2}\right\}\right)=r^{*}(X)=4$, contradicting that $g_{1}, g_{2} \notin \mathrm{cl}^{*}(X)$.

Next we handle one particularly awkward case that arises.

4.4.6. Suppose $C_{1}^{*}=\left\{d_{0}, x, c_{1}, d_{1}\right\}$ and $C_{2}^{*}=\left\{d_{0}, x, c_{2}, d_{2}\right\}$ and let $X=$ $C_{1}^{*} \cup C_{2}^{*}$. If $C_{0}=\left\{c_{1}, c_{2}, d_{0}, e\right\}$ is a circuit, for some $e \in E(M)-X$, and $C_{3}^{*}=\left\{d_{1}, e, c_{2}, f_{1}\right\}$ and $C_{4}^{*}=\left\{d_{2}, e, c_{1}, f_{2}\right\}$ are cocircuits, for $f_{1}, f_{2} \in$ $E(M)-(X \cup e)$, then the lemma holds.

Subproof. Since $M \backslash d_{0} \backslash d_{1} \backslash d_{2} / c_{1} / c_{2}$ has an $N$-minor, it follows that $M \backslash d_{1} \backslash d_{2} \backslash e / c_{1} / f_{1}$ and $M \backslash d_{1} \backslash d_{2} \backslash e / c_{2} / f_{2}$ have $N$-minors. Let $C_{3}$ be the 4-element circuit containing $\left\{c_{1}, f_{1}\right\}$, and let $C_{4}$ be the 4 -element circuit containing $\left\{c_{2}, f_{2}\right\}$.

Suppose $f_{1} \neq f_{2}$. By orthogonality with $C_{3}^{*}$ and $C_{4}^{*}$, the circuit $C_{3}$ meets $\left\{e, c_{2}, d_{1}\right\}$ and $\left\{e, d_{2}, f_{2}\right\}$. If $e \in C_{3}$, then $C_{2}^{*} \cap C_{3}=\emptyset$, so, by orthogonality with $C_{1}^{*}$, we see that $C_{3}=\left\{c_{1}, f_{1}, e, d_{1}\right\}$. On the other hand, if $e \notin C_{3}$ and $C_{3}$ meets $C_{2}^{*}$, then $C_{3}$ contains both $c_{2}$ and $d_{2}$, which then contradicts orthogonality with $C_{1}^{*}$. So when $e \notin C_{3}$, the sets $C_{2}^{*}$ and $C_{3}$ are disjoint, in which case $\left\{d_{1}, f_{2}\right\} \subseteq C_{3}$. Now $C_{3}=\left\{c_{1}, f_{1}, d_{1}, p_{1}\right\}$ for some $p_{1} \in\left\{e, f_{2}\right\}$, and, by symmetry, $C_{4}=\left\{c_{2}, f_{2}, d_{2}, p_{2}\right\}$ for some $p_{2} \in\left\{e, f_{1}\right\}$.

Let $Y=X \cup\left\{e, f_{1}, f_{2}\right\}$. Next, we claim that $x \notin \operatorname{cl}(Y-x)$. Observe that $e \in \operatorname{cl}(X-x)$, due to the circuit $C_{0}$. If $e \in\left\{p_{1}, p_{2}\right\}$, then $r(Y-x)=$ $r(X-x) \leq 5$, due to the circuits $C_{3}$ and $C_{4}$. Otherwise, $C_{3}=\left\{c_{1}, f_{1}, d_{1}, f_{2}\right\}$ and $C_{4}=\left\{c_{2}, f_{2}, d_{2}, f_{1}\right\}$, in which case $\left\{f_{1}, f_{2}, c_{1}, c_{2}, d_{0}\right\}$ spans $Y-x$. So $r(Y-x) \leq 5$. Since $r^{*}(Y-x) \leq 5$, we have $\lambda(Y-x) \leq 5+5-8=2$. Now, if $x \in \operatorname{cl}(Y-x)$, then $\lambda(Y) \leq 1$, so $|E(M)| \leq 10$; a contradiction.

Consider the 4-element circuit $C_{1}$ containing $\left\{c_{1}, x\right\}$. By orthogonality with $C_{2}^{*}$, this circuit meets $\left\{d_{0}, d_{2}, c_{2}\right\}$. If $d_{0} \in C_{1}$, then, by orthogonality 
with $C_{4}^{*}$, the final element is in $\left\{e, d_{2}, f_{2}\right\}$, in which case $x \in \operatorname{cl}(Y-x)$; a contradiction. If $c_{2} \in C_{1}$, then, by orthogonality with $C_{3}^{*}$ and $C_{4}^{*}$, the final element is $e$; again, we obtain the contradiction that $x \in \operatorname{cl}(Y-x)$. So $d_{2} \in C_{1}$. Let $C_{1}=\left\{c_{1}, x, d_{2}, g_{1}\right\}$, for some $g_{1} \in E(M)-\left\{c_{1}, x, d_{2}\right\}$. Note that $g_{1} \notin Y$, since $x \notin \operatorname{cl}(Y-x)$. By symmetry, there is a circuit $C_{2}=\left\{c_{2}, x, d_{1}, g_{2}\right\}$ where $g_{2} \in E(M)-Y$. If $g_{1}=g_{2}$, then $\left\{c_{1}, c_{2}, x, d_{1}, d_{2}\right\}$ contains a circuit, which cannot contain $x$ since $x \notin \operatorname{cl}(Y-x)$. But then $\left\{c_{1}, c_{2}, d_{1}, d_{2}\right\}$ is a circuit, so the lemma holds by 4.4.4. So we may assume that $g_{1} \neq g_{2}$.

As $M \backslash d_{0} / c_{1} / x$ has an $N$-minor, the pair $\left\{d_{0}, g_{1}\right\}$ is $N$-deletable. Thus $\left\{d_{0}, g_{1}\right\}$ is contained in a 4 -element cocircuit $C_{5}^{*}$. By orthogonality with $C_{1}$ and $C_{0}$, the cocircuit $C_{5}^{*}$ meets $\left\{c_{1}, x, d_{2}\right\}$ and $\left\{c_{1}, c_{2}, e\right\}$.

Consider when $c_{1} \notin C_{5}^{*}$. Then, by orthogonality with $C_{2}$, either $C_{5}^{*}=\left\{d_{0}, g_{1}, d_{2}, e\right\}$ or $C_{5}^{*}=\left\{d_{0}, g_{1}, x, c_{2}\right\}$. Suppose $C_{5}^{*}=\left\{d_{0}, g_{1}, d_{2}, e\right\}$. Then $M \backslash d_{0} \backslash d_{1} \backslash d_{2} / c_{1} / c_{2} / g_{1}$ has an $N$-minor, so there is a 4 -element circuit $C_{5}$ containing $\left\{c_{2}, g_{1}\right\}$. If $x \notin C_{5}$, then $C_{5}=\left\{c_{2}, g_{1}, d_{0}, d_{1}\right\}$ or $C_{5}=$ $\left\{c_{2}, g_{1}, d_{2}, e\right\}$ by orthogonality with $C_{3}^{*}$ and $C_{4}^{*}$, in which case $g_{1} \in \operatorname{cl}(Y-x)$. As $\lambda(Y-x)=2$, we have $\lambda\left((Y-x) \cup g_{1}\right) \leq 1$ and $|E(M)| \leq 10$; a contradiction. So $x \in C_{5}$, in which case $C_{5}=\left\{c_{2}, g_{1}, x, d_{1}\right\}$, and $C_{2} \cup C_{5}$ is a 5 -element plane that intersects $C_{1}^{*}$ in two elements; a contradiction. Now suppose $C_{5}^{*}=\left\{d_{0}, g_{1}, x, c_{2}\right\}$. Then $C_{2}^{*} \cup C_{5}^{*}$ is a 5-element coplane that intersects the circuit $C_{0}$ in two elements; a contradiction.

So now we may assume $\left\{d_{0}, g_{1}, c_{1}\right\} \subseteq C_{5}^{*}$. By orthogonality with $C_{3}$, the final element of $C_{5}^{*}$ is in $\left\{f_{1}, d_{1}, p_{1}\right\}$. If $d_{1} \in C_{5}^{*}$, then $C_{1}^{*} \cup C_{5}^{*}$ is a 5-element coplane that intersects the circuit $C_{0}$ in two elements; a contradiction. By orthogonality with $C_{3}$, either $C_{5}^{*}=\left\{d_{0}, g_{1}, c_{1}, f_{1}\right\}$, or $C_{5}^{*}=\left\{d_{0}, g_{1}, c_{1}, e\right\}$ and $p_{1}=e$. Consider the former case, where $C_{5}^{*}=\left\{d_{0}, g_{1}, c_{1}, f_{1}\right\}$. As $M \backslash d_{0} / c_{1} / x$ has an $N$-minor, $M \backslash d_{0} \backslash g_{1} / x$ has an $N$-minor, and thus $\left\{f_{1}, x\right\}$ is $N$-contractible. Now there is a 4-element circuit $C_{6}$ containing $\left\{f_{1}, x\right\}$, which meets $\left\{d_{0}, d_{1}, c_{1}\right\}$ and $\left\{d_{0}, d_{2}, c_{2}\right\}$, by orthogonality. If $d_{0} \notin C_{6}$, then $C_{6}=$ $\left\{f_{1}, x, c_{1}, d_{2}\right\}$ or $C_{6}=\left\{f_{1}, x, c_{2}, d_{1}\right\}$, by orthogonality with $C_{3}^{*}$. But then $C_{1} \cup f_{1}$ or $C_{2} \cup f_{1}$, respectively, is a 5-element plane that intersects $C_{1}^{*}$ in two elements; a contradiction. So $d_{0} \in C_{6}$. By orthogonality with $C_{3}^{*}$ and $C_{4}^{*}$, we have $C_{6}=\left\{f_{1}, x, d_{0}, d_{1}\right\}$ or $C_{6}=\left\{f_{1}, x, d_{0}, c_{2}\right\}$. In either case, $x \in \operatorname{cl}(Y-x)$; a contradiction. So $C_{5}^{*}=\left\{d_{0}, g_{1}, c_{1}, e\right\}$ and $C_{3}=\left\{c_{1}, f_{1}, d_{1}, e\right\}$. By circuit elimination on $C_{3}$ and $C_{0}$, there is a circuit contained in $\left\{c_{1}, f_{1}, d_{1}, d_{0}, c_{2}\right\}$. By orthogonality with $C_{4}^{*}$, this circuit is $\left\{f_{1}, d_{1}, d_{0}, c_{2}\right\}$, but then it intersects $C_{5}^{*}$ in a single element; a contradiction.

Now suppose $f_{1}=f_{2}=f$. Let $Y=X \cup\{e, f\}$. By orthogonality, $C_{3}$ meets $\left\{d_{1}, d_{0}, x\right\}$ and $\left\{d_{1}, e, c_{2}\right\}$. If $d_{1} \notin C_{3}$, then by orthogonality with $C_{2}^{*}$, we have $c_{2} \in C_{3}$. But if $C_{3}=\left\{c_{1}, f, d_{0}, c_{2}\right\}$, then $C_{0} \cup C_{3}$ is a 5-element plane that intersects $C_{1}^{*}$ in two elements, contradicting orthogonality. So $C_{3}=\left\{c_{1}, f, x, c_{2}\right\}$ when $d_{1} \notin C_{3}$. On the other hand, if $d_{1} \in C_{3}$, then by 
orthogonality with $C_{2}^{*}$, either $C_{3}=\left\{c_{1}, f, d_{1}, e\right\}$, or $C_{3}=\left\{c_{1}, f, d_{1}, g_{3}\right\}$ for some $g_{3} \in E(M)-Y$.

We claim that if $\left\{c_{1}, f, x, c_{2}\right\}$ is a circuit, then the lemma holds. Suppose $C_{3}=\left\{c_{1}, f, x, c_{2}\right\}$ is a circuit. As $M \backslash d_{0} \backslash d_{1} \backslash d_{2} / c_{1} / c_{2}$ has an $N$-minor and $\{f, x\}$ is a parallel pair in this matroid, $M \backslash d_{0} \backslash d_{1} \backslash d_{2} \backslash x$ has an $N$-minor. This matroid has $c_{1}$ and $c_{2}$ as coloops, so $M \backslash X$ has an $N$-minor. Now, since $M \backslash\left(X-\left\{d_{1}, d_{2}\right\}\right)$ has an $N$-minor, we see that $\left\{d_{1}, d_{2}\right\}$ is $N$-contractible, so this pair is contained in a 4 -element circuit $C_{4}$. If $C_{4}=\left\{d_{1}, d_{2}, c_{1}, c_{2}\right\}$, then the lemma holds by 4.4.4. So we may assume, by orthogonality with $C_{1}^{*}$ and $C_{3}^{*}$, that $C_{4}$ meets $\left\{x, d_{0}\right\}$ and $\{e, f\}$. It now follows that $d_{1} \in \operatorname{cl}\left(Y-d_{1}\right)$, so $r(Y) \leq 5$. Moreover, as $\left\{e, d_{0}\right\}$ and $\{f, x\}$ are parallel pairs in the matroid $M \backslash d_{1} \backslash d_{2} / c_{1} / c_{2}$, the matroid $M \backslash d_{1} \backslash d_{2} \backslash e \backslash f$ has an $N$-minor. If $\left\{d_{1}, d_{2}, e, f\right\}$ is a cocircuit, then it follows that $r^{*}(Y) \leq 4$; a contradiction. So $\left\{d_{1}, d_{2}, e, f\right\}$ is coindependent, and hence $r_{M \backslash d_{1} \backslash d_{2} \backslash e \backslash f}^{*}\left(\left\{c_{1}, c_{2}, x, d_{0}\right\}\right)=1$, implying the pair $\left\{x, d_{0}\right\}$ is $N$-contractible. Now $r\left(Y-\left\{x, d_{0}\right\}\right) \leq 5$ and $r^{*}\left(Y-\left\{x, d_{0}\right\}\right)=$ 4 , so $\lambda_{M / x}\left(Y-\left\{x, d_{0}\right\}\right)=2$. Since $d_{0} \in \operatorname{cl}_{M / x}\left(Y-\left\{x, d_{0}\right\}\right)$, (ii) holds by the dual of Lemma 4.2. Henceforth, we may assume that $\left\{c_{1}, f, x, c_{2}\right\}$ is not a circuit.

Now $C_{3}=\left\{c_{1}, f, d_{1}, e\right\}$ or $C_{3}=\left\{c_{1}, f, d_{1}, g_{3}\right\}$ for some $g_{3} \in E(M)-Y$. By symmetry, $C_{4}=\left\{c_{2}, f, d_{2}, e\right\}$ or $C_{4}=\left\{c_{2}, f, d_{2}, g_{4}\right\}$ for some $g_{4} \in E(M)-$ $Y$. If $C_{3}=\left\{c_{1}, f, d_{1}, e\right\}$ and $C_{4}=\left\{c_{2}, f, d_{2}, e\right\}$, then $x \notin \operatorname{cl}(Y-x)$, and $\left\{c_{1}, x, d_{2}\right\} \subseteq C_{1}$. In this case, by using a similar argument as in the case that $f_{1} \neq f_{2}$, it follows that $|E(M)| \leq 10$; we omit the details. So we may assume, without loss of generality, that $C_{3}=\left\{c_{1}, f, d_{1}, g_{3}\right\}$ for some $g_{3} \in E(M)-Y$.

Recall also the 4-element circuit $C_{1}$ containing $\left\{c_{1}, x\right\}$. We claim that either $C_{1}=\left\{c_{1}, x, d_{0}, d_{2}\right\}$ or $C_{1}=\left\{c_{1}, x, d_{2}, g_{1}\right\}$ for some $g_{1} \in E(M)-X$. If $c_{2} \in C_{1}$, then, by orthogonality with $C_{3}^{*}$ and $C_{4}^{*}$, either $C_{1}=\left\{c_{1}, x, c_{2}, e\right\}$ or $C_{1}=\left\{c_{1}, x, c_{2}, f\right\}$. We have already argued that the latter is not a circuit. In the former case, $C_{0} \cup x$ is a 5-element plane that intersects $C_{3}^{*}$ in two elements; a contradiction. By orthogonality with $C_{2}^{*}$, it now follows that either $d_{0} \in C_{1}$ or $d_{2} \in C_{1}$. If $d_{0} \in C_{1}$, then $C_{1}=\left\{c_{1}, x, d_{0}, d_{2}\right\}$, by orthogonality with $C_{3}^{*}$ and $C_{4}^{*}$. Otherwise, $d_{2} \in C_{1}$, and $C_{1}$ intersects $C_{4}^{*}$ in at most one element. Thus, by orthogonality, either $C_{1}=\left\{c_{1}, x, d_{0}, d_{2}\right\}$ or $C_{1}=\left\{c_{1}, x, d_{2}, g_{1}\right\}$ for some $g_{1} \in E(M)-X$. Similarly, either $C_{2}=$ $\left\{c_{2}, x, d_{0}, d_{1}\right\}$ or $C_{2}=\left\{c_{2}, x, d_{1}, g_{2}\right\}$ for $g_{2} \in E(M)-X$.

Suppose $C_{1}=\left\{c_{1}, x, d_{0}, d_{2}\right\}$ and $C_{2}=\left\{c_{2}, x, d_{0}, d_{1}\right\}$. Then $r(X) \leq 4$, and $r^{*}(X) \leq 4$, so $X$ is 3 -separating, and (i) holds by 4.4.3. So we may assume that, for some $\{i, j\}=\{1,2\}$, we have $C_{i}=\left\{c_{i}, x, d_{j}, g_{i}\right\}$.

Suppose that $C_{4}=\left\{c_{2}, f, d_{2}, e\right\}$. Recall that $M \backslash d_{1} \backslash d_{2} \backslash e / c_{1} / c_{2}$ has an $N$-minor. Due to the cocircuit $C_{3}^{*}$, it follows that $M \backslash d_{2} \backslash e / c_{1} / f$ has an $N$ minor. As $\left\{d_{1}, g_{3}\right\}$ is a parallel pair in this matroid, $M \backslash g_{3} \backslash e$ also has an $N$ minor, so there is a 4-element cocircuit $C_{5}^{*}$ containing $\left\{g_{3}, e\right\}$. As $C_{5}^{*}$ meets the circuits $C_{0}, C_{3}$, and $C_{4}$, the cocircuit also contains an element from each 
of $\left\{d_{0}, c_{1}, c_{2}\right\},\left\{d_{1}, c_{1}, f\right\}$, and $\left\{d_{2}, c_{2}, f\right\}$, by orthogonality. It follows that $C_{5}^{*} \subseteq Y \cup g_{3}$, so $g_{3} \in \mathrm{cl}^{*}(Y)$. If $r(Y) \leq 5$, then, as $r^{*}(Y) \leq 5$ and $g_{3} \in \operatorname{cl}(Y)$, due to the circuit $C_{3}$, we have $\lambda\left(Y \cup g_{3}\right) \leq 1$, implying $|E(M)| \leq 10$; a contradiction. So $r(Y) \geq 6$. In particular, if, for some $\{i, j\}=\{1,2\}$, we have $C_{j}=\left\{c_{j}, x, d_{0}, d_{i}\right\}$, then we obtain the contradiction that $r(Y) \leq 5$.

So $C_{1}=\left\{c_{1}, x, d_{2}, g_{1}\right\}$ and $C_{2}=\left\{c_{2}, x, d_{1}, g_{2}\right\}$. Moreover, if $g_{3} \in\left\{g_{1}, g_{2}\right\}$, then, after circuit elimination on $C_{3}$ and either $C_{1}$ or $C_{2}$, it follows that $r(Y) \leq 5$; a contradiction. By a similar argument, $g_{1} \neq g_{2}$. So we may assume that $g_{1}, g_{2}$, and $g_{3}$ are distinct. Since $M \backslash d_{2} \backslash e / c_{1} / f$ has an $N$-minor, and $\left\{g_{3}, d_{1}\right\}$ is a parallel pair in this matroid, $\left\{g_{3}, d_{2}\right\}$ is $N$-deletable. So there is a 4-element cocircuit $C_{6}^{*}$ containing $\left\{g_{3}, d_{2}\right\}$. By orthogonality with $C_{1}$ and $C_{4}$, the cocircuit contains an element in $\left\{g_{1}, x, c_{1}\right\}$ and an element in $\left\{c_{2}, e, f\right\}$. If $C_{6}^{*}$ meets $\left\{c_{2}, e\right\}$, then, by orthogonality with $C_{0}$, the other element is $c_{1}$. Then, by orthogonality with $C_{2}$, we have $C_{6}^{*}=\left\{g_{3}, d_{2}, c_{1}, e\right\}$, in which case $C_{3}^{*} \cup g_{3}$ is a 5-element coplane intersecting $C_{0}$ in two elements; a contradiction. So $f \in C_{6}^{*}$. Then, the final element of $C_{6}^{*}$ is in $\left\{g_{1}, x, c_{1}\right\}$, and by orthogonality $C_{6}^{*}$ avoids $C_{0}$ and $C_{2}$; so $C_{6}^{*}=\left\{g_{3}, d_{2}, f, g_{1}\right\}$.

Now $M \backslash d_{2} \backslash e / c_{1} / f$ has an $N$-minor, and $\left\{d_{1}, g_{3}\right\}$ is a parallel pair in this matroid, due to $C_{3}$, so $M \backslash g_{3} \backslash d_{2} \backslash e / c_{1}$ has an $N$-minor. As $\left\{f, g_{1}\right\}$ is a series pair in this matroid, due to $C_{6}^{*}$, the matroid $M \backslash e / c_{1} / g_{1}$ has an $N$-minor. As $\left\{x, d_{2}\right\}$ is a parallel pair in this matroid, due to $C_{1}$, the pair $\{x, e\}$ is $N$-deletable. So there is a 4-element cocircuit $C_{7}^{*}$ containing $\{x, e\}$, and meeting $\left\{c_{1}, d_{2}, g_{1}\right\},\left\{c_{2}, d_{1}, g_{2}\right\}$ by orthogonality with $C_{1}$ and $C_{2}$. By orthogonality with $C_{3}$ and $C_{0}$, this cocircuit is either $\left\{x, e, c_{1}, d_{1}\right\}$, $\left\{x, e, d_{2}, c_{2}\right\}$, or $\left\{x, e, g_{1}, c_{2}\right\}$. If $C_{7}^{*}=\left\{x, e, c_{1}, d_{1}\right\}$ or $C_{7}^{*}=\left\{x, e, d_{2}, c_{2}\right\}$, then $C_{1}^{*} \cup e$ or $C_{2}^{*} \cup e$ is a 5-element coplane intersecting $C_{1}$ or $C_{2}$ in two elements, respectively; a contradiction. So $C_{7}^{*}=\left\{x, e, c_{2}, g_{1}\right\}$. Then, by cocircuit elimination with $C_{2}^{*}$, there is a cocircuit $C_{8}^{*}$ contained in $\left\{e, g_{1}, x, d_{0}, d_{2}\right\}$. By orthogonality with $C_{2}$, we have $C_{8}^{*}=\left\{d_{0}, d_{2}, e, g_{1}\right\}$. Now, both $\left\{x, c_{2}\right\}$ and $\left\{e, g_{1}\right\}$ are series pairs in $M \backslash d_{0} \backslash d_{2}$, so $M / x / e$ has an $N$-minor. Consider the 4-element circuit containing $\{e, x\}$. By orthogonality with $C_{1}^{*}, C_{2}^{*}, C_{3}^{*}$, and $C_{4}^{*}$, the circuit is either $\left\{e, x, f, d_{0}\right\},\left\{e, x, d_{1}, d_{2}\right\}$, or $\left\{e, x, c_{1}, c_{2}\right\}$. In the first two cases, the circuit intersects $C_{6}^{*}$ in a single element, while in the latter case the circuit intersects $C_{8}^{*}$ in a single element; a contradiction.

So in what follows, we may assume that $C_{3}=\left\{c_{1}, f, d_{1}, g_{3}\right\}$ and $C_{4}=$ $\left\{c_{2}, f, d_{2}, g_{4}\right\}$, for $g_{3}, g_{4} \in E(M)-Y$. By symmetry, we may also assume $C_{1}=\left\{c_{1}, x, d_{2}, g_{1}\right\}$. Suppose $C_{2}=\left\{c_{2}, x, d_{0}, d_{1}\right\}$. Since $M \backslash d_{1} \backslash e / c_{2} / f$ has an $N$-minor, and $\left\{d_{2}, g_{4}\right\}$ is a parallel pair in this matroid, $\left\{g_{4}, d_{1}\right\}$ is $N$ deletable. Consider the 4-element cocircuit containing $\left\{g_{4}, d_{1}\right\}$. By orthogonality with $C_{2}$ and $C_{4}$, the cocircuit meets $\left\{c_{2}, x, d_{0}\right\}$ and $\left\{c_{2}, d_{2}, f\right\}$. If the cocircuit contains $c_{2}$, then the final element is $c_{1}$, by orthogonality with $C_{3}$ and $C_{0}$. But then the cocircuit intersects $C_{1}$ in a single element, contradicting orthogonality. So the cocircuit contains an element in $\left\{x, d_{0}\right\}$ and an element in $\left\{d_{2}, f\right\}$. By orthogonality with $C_{1}$, the cocircuit is either $\left\{g_{4}, d_{1}, x, d_{2}\right\}$ or $\left\{g_{4}, d_{1}, d_{0}, f\right\}$. But the former intersects $C_{3}$ in a single 
element, and the latter intersects $C_{0}$ in a single element. From this contradiction, we deduce that $C_{1}=\left\{c_{1}, x, d_{2}, g_{1}\right\}$ and $C_{2}=\left\{c_{2}, x, d_{1}, g_{2}\right\}$ for $g_{1}, g_{2} \in E(M)-Y$.

Now suppose $C_{1}=\left\{c_{1}, x, d_{2}, g_{1}\right\}, C_{2}=\left\{c_{2}, x, d_{1}, g_{2}\right\}, C_{3}=\left\{c_{1}, f, d_{1}, g_{3}\right\}$, and $C_{4}=\left\{c_{2}, f, d_{2}, g_{4}\right\}$, for $g_{1}, g_{2}, g_{3}, g_{4} \in E(M)-Y$. Since $M \backslash d_{0} / c_{1} / x$ has an $N$-minor and $\left\{g_{1}, d_{2}\right\}$ is a parallel pair in this matroid, $\left\{g_{1}, d_{0}\right\}$ is $N$-deletable. Consider the 4 -element cocircuit $C_{5}^{*}$ containing $\left\{g_{1}, d_{0}\right\}$. This cocircuit meets $\left\{c_{1}, x, d_{2}\right\}$ and $\left\{c_{1}, c_{2}, e\right\}$ by orthogonality with $C_{1}$ and $C_{0}$. Also, $\left|C_{5}^{*} \cap C_{1}^{*}\right| \neq 3$, for otherwise $C_{1}^{*} \cup g_{1}$ is a 5-element coplane that intersects $C_{0}$ or $C_{1}$ in two elements; a contradiction. Similarly, $\left|C_{5}^{*} \cap C_{2}^{*}\right| \neq 3$.

Suppose $g=g_{1}=g_{2}$. Then $C_{5}^{*}$ also meets $\left\{c_{2}, x, d_{1}\right\}$, and it follows that $C_{5}^{*}=\left\{g, d_{0}, c_{1}, c_{2}\right\}$ or $C_{5}^{*}=\left\{g, d_{0}, x, e\right\}$. In the former case, it follows that $g=g_{3}=g_{4}$, so $C_{3}=\left\{c_{1}, f, d_{1}, g\right\}$ and $C_{4}=\left\{c_{2}, f, d_{2}, g\right\}$. Now $r^{*}(Y \cup g) \leq 5$ and $r(Y \cup g) \leq 5$, so $\lambda(Y \cup g) \leq 1$ and $|E(M)| \leq 10$; a contradiction. So $C_{5}^{*}=\left\{g, d_{0}, x, e\right\}$. By circuit elimination on $C_{1}$ and $C_{2}$, the set $\left\{c_{1}, d_{1}, x, c_{2}, d_{2}\right\}$ contains a circuit. By orthogonality with $C_{5}^{*}$, we see that $C_{1}^{*} \triangle C_{2}^{*}=\left\{c_{1}, d_{1}, c_{2}, d_{2}\right\}$ is a circuit, so the lemma holds by 4.4.4. So we may assume that $g_{1} \neq g_{2}$.

Now suppose $g^{\prime}=g_{3}=g_{4}$. Since $M \backslash d_{1} \backslash e / c_{1}$ has an $N$-minor, so does $M \backslash e / c_{1} / f$, and it follows that $\left\{e, g^{\prime}\right\}$ is $N$-deletable. Thus there is a 4 element cocircuit $C^{*}$ containing $\left\{e, g^{\prime}\right\}$. If $C^{*}=\left\{e, g^{\prime}, c_{1}, c_{2}\right\}$, then, by orthogonality with $C_{1}$ and $C_{2}$, we have $g^{\prime}=g_{1}=g_{2}$; a contradiction. Now, if $c_{1} \in C^{*}$, then by orthogonality with $C_{3}$, the final element is either $d_{2}$ or $f$; but then $C_{4}^{*} \cup g^{\prime}$ is a 5-element coplane intersecting $C_{0}$ in two elements; a contradiction. So $c_{1} \notin C^{*}$ and, similarly, $c_{2} \notin C^{*}$; hence $d_{0} \in C^{*}$. By orthogonality with $C_{3}$ and $C_{4}$, we have $C^{*}=\left\{d_{0}, e, f, g^{\prime}\right\}$. Now by circuit elimination on $C_{3}$ and $C_{4}$, there is a circuit contained in $\left\{c_{1}, d_{1}, f, c_{2}, d_{2}\right\}$. By orthogonality with $C^{*}$, we see that $C_{1}^{*} \triangle C_{2}^{*}=\left\{c_{1}, d_{1}, c_{2}, d_{2}\right\}$ is a circuit, so the lemma holds by 4.4.4. So we may assume that $g_{3} \neq g_{4}$.

We return to the 4 -element cocircuit $C_{5}^{*}$ containing $\left\{g_{1}, d_{0}\right\}$ and meeting $\left\{c_{1}, x, d_{2}\right\}$ and $\left\{c_{1}, c_{2}, e\right\}$. Consider when $c_{1} \notin C_{5}^{*}$. Then, by orthogonality with $C_{2}$, either $C_{5}^{*}=\left\{d_{0}, g_{1}, d_{2}, e\right\}$ or $C_{5}^{*}=\left\{d_{0}, g_{1}, x, c_{2}\right\}$. But if $C_{5}^{*}=$ $\left\{d_{0}, g_{1}, x, c_{2}\right\}$, then $C_{2}^{*} \cup g_{1}$ is a 5-element coplane that intersects $C_{0}$ in two elements; a contradiction. So $C_{5}^{*}=\left\{d_{0}, g_{1}, d_{2}, e\right\}$. Similarly, there is a 4-element cocircuit $C_{6}^{*}$ containing $\left\{d_{0}, g_{2}\right\}$ where either $c_{2} \in C_{6}^{*}$ or $C_{6}^{*}=\left\{d_{0}, g_{2}, d_{1}, e\right\}$.

Suppose $C_{5}^{*}=\left\{d_{0}, g_{1}, d_{2}, e\right\}$ and $C_{6}^{*}=\left\{d_{0}, g_{2}, d_{1}, e\right\}$. Then, by cocircuit elimination, there is a cocircuit contained in $\left\{g_{1}, g_{2}, d_{1}, d_{2}, e\right\}$. By orthogonality with $C_{0}$, this cocircuit is $\left\{g_{1}, g_{2}, d_{1}, d_{2}\right\}$. Now by orthogonality we have $g_{2}=g_{3}$ and $g_{1}=g_{4}$, so $C_{3}=\left\{c_{1}, f, d_{1}, g_{2}\right\}$ and $C_{4}=\left\{c_{2}, f, d_{2}, g_{1}\right\}$. As $M \backslash d_{1} \backslash d_{2} \backslash e / c_{1} / c_{2}$ has an $N$-minor, and $\left\{g_{1}, g_{2}\right\}$ is a series pair in this matroid, $M \backslash e / c_{2} / g_{1}$ has an $N$-minor. As $\left\{d_{2}, f\right\}$ is a parallel pair in this matroid, $M \backslash e \backslash f / g_{1}$ also has an $N$-minor. Then $\left\{d_{1}, c_{2}\right\}$ is a series pair in the latter matroid, so $\left\{g_{1}, d_{1}\right\}$ is $N$-contractible. So there is a 4 -element 
circuit containing $\left\{g_{1}, d_{1}\right\}$. By orthogonality with $C_{5}^{*}$ and $C_{1}^{*}$, this circuit meets $\left\{d_{0}, d_{2}, e\right\}$ and $\left\{d_{0}, x, c_{1}\right\}$. If the circuit contains $d_{0}$, then, by orthogonality with $C_{2}^{*}$ and $C_{4}^{*}$, it is $\left\{g_{1}, d_{1}, d_{0}, c_{2}\right\}$. Otherwise, when the circuit does not contain $d_{0}$, then, by orthogonality with $C_{4}^{*}$ and $C_{3}^{*}$, it is $\left\{g_{1}, d_{1}, e, c_{1}\right\}$. In either case, $g_{1} \in \operatorname{cl}\left(C_{0} \cup d_{1}\right)$, and it follows that $r\left(Y \cup\left\{g_{1}, g_{2}\right\}\right) \leq 5$. As $r^{*}\left(Y \cup\left\{g_{1}, g_{2}\right\}\right) \leq 5$, we have $\lambda\left(Y \cup\left\{g_{1}, g_{2}\right\}\right)=0$, implying $|E(M)|=10$; a contradiction. We deduce that either $c_{1} \in C_{5}^{*}$ or $c_{2} \in C_{6}^{*}$.

By symmetry, we may now assume that $\left\{d_{0}, g_{1}, c_{1}\right\} \subseteq C_{5}^{*}$. First we assume that $g_{1} \neq g_{3}$. By orthogonality with $C_{3}$, the final element of $C_{5}^{*}$ is in $\left\{f, d_{1}, g_{3}\right\}$. If $d_{1} \in C_{5}^{*}$, then $C_{1}^{*} \cup C_{5}^{*}$ is a 5 -element coplane that intersects the circuit $C_{0}$ in two elements; a contradiction. So either $C_{5}^{*}=\left\{d_{0}, g_{1}, c_{1}, f\right\}$, or $C_{5}^{*}=\left\{d_{0}, g_{1}, c_{1}, g_{3}\right\}$.

Suppose $C_{5}^{*}=\left\{d_{0}, g_{1}, c_{1}, f\right\}$. As $M \backslash d_{0} / c_{1} / x$ has an $N$-minor, $M \backslash d_{0} \backslash g_{1} / x$ has an $N$-minor, and thus $\{f, x\}$ is $N$-contractible. So there is a 4 -element circuit $C_{6}$ containing $\{f, x\}$, which meets $\left\{d_{0}, d_{1}, c_{1}\right\}$ and $\left\{d_{0}, d_{2}, c_{2}\right\}$, by orthogonality. If $d_{0} \notin C_{6}$, then $C_{6}=\left\{f, x, c_{1}, c_{2}\right\}$ or $C_{6}=\left\{f, x, d_{1}, d_{2}\right\}$, by orthogonality with $C_{3}^{*}$ and $C_{4}^{*}$. By orthogonality with $C_{5}^{*}$, we have $C_{6}=\left\{f, x, c_{1}, c_{2}\right\}$. But $\left\{c_{1}, f, x, c_{2}\right\}$ is not a circuit, by an earlier claim. So $d_{0} \in C_{6}$. By orthogonality with $C_{3}^{*}$ and $C_{4}^{*}$, we now have $C_{6}=$ $\left\{f, x, d_{0}, e\right\}$. By cocircuit elimination on $C_{3}^{*}$ and $C_{5}^{*}$, there is a cocircuit contained in $\left\{c_{1}, d_{2}, e, d_{0}, g_{1}\right\}$. By orthogonality with $C_{3}$, the cocircuit is $C_{7}^{*}=\left\{d_{2}, e, d_{0}, g_{1}\right\}$. Now, by circuit elimination on $C_{0}$ and $C_{6}$, there is a circuit contained in $\left\{e, c_{1}, c_{2}, f, x\right\}$; by orthogonality with $C_{7}^{*}$, this circuit is $\left\{c_{1}, c_{2}, f, x\right\}$. But, as argued earlier, this is not a circuit.

Now suppose $C_{5}^{*}=\left\{d_{0}, g_{1}, c_{1}, g_{3}\right\}$. Since $M \backslash d_{1} / c_{1} / c_{2}$ has an $N$-minor, it follows that $M \backslash e \backslash d_{1} / c_{1}$ has an $N$-minor, so $M \backslash e / c_{1} / f$ has an $N$-minor, and finally $\left\{e, g_{3}\right\}$ is $N$-deletable. So there is a 4-element cocircuit $C_{7}^{*}$ containing $\left\{e, g_{3}\right\}$. By orthogonality with $C_{3}$ and $C_{0}$, this cocircuit meets $\left\{d_{1}, c_{1}, f\right\}$ and $\left\{c_{1}, c_{2}, d_{0}\right\}$. If it contains $c_{1}$, so that $\left\{e, g_{3}, c_{1}\right\} \subseteq C_{7}^{*}$, then by orthogonality with $C_{1}$, the final element is in $\left\{x, d_{2}, g_{1}\right\}$. By orthogonality with $C_{2}$ and $C_{4}$, it follows that $C_{7}^{*}=\left\{e, g_{3}, c_{1}, g_{1}\right\}$. But then $C_{5}^{*} \cup C_{7}^{*}$ is a 5-element coplane that intersects $C_{3}$ in two elements; a contradiction. So $c_{1} \notin C_{7}^{*}$, and thus $C_{7}^{*}$ meets $\left\{c_{2}, d_{0}\right\}$ and $\left\{d_{1}, f\right\}$, by orthogonality with $C_{0}$ and $C_{3}$. But this contradicts orthogonality with $C_{1}$, or with $C_{4}$.

Next we assume that $g_{1}=g_{3}$. Suppose also that $g_{2}=g_{4}$. Recall that $\left\{d_{0}, g_{1}, c_{1}\right\} \subseteq C_{5}^{*}$. By orthogonality, $C_{5}^{*}$ does not meet $C_{2}$ or $C_{4}$, so either $C_{5}^{*}=\left\{d_{0}, g_{1}, c_{1}, e\right\}$ or $C_{5}^{*}=\left\{d_{0}, g_{1}, c_{1}, h_{1}\right\}$ for some $h_{1} \in E(M)-(Y \cup$ $\left.\left\{g_{1}, g_{2}\right\}\right)$. Suppose $C_{5}^{*}=\left\{d_{0}, g_{1}, c_{1}, e\right\}$ and observe that $r^{*}\left(Y \cup g_{1}\right) \leq 5$. We claim that $M / d_{0} / f$ has an $N$-minor. As $M \backslash d_{1} \backslash d_{2} \backslash e / c_{1}$ has an $N$ minor and $\left\{f, c_{2}\right\}$ is a series pair in this matroid, $M \backslash d_{2} \backslash e / c_{1} / f$ has an $N$-minor. Due to $C_{3}$, the latter matroid has the parallel pair $\left\{d_{1}, g_{1}\right\}$, so $M \backslash g_{1} \backslash d_{2} \backslash e / f$ has an $N$-minor. Then $\left\{d_{0}, c_{1}\right\}$ is a series pair in this matroid, due to $C_{5}^{*}$, so $M \backslash g_{1} \backslash d_{2} / d_{0} / f$ has an $N$-minor, thus showing the claim. Now $\left\{d_{0}, f\right\}$ is contained in a 4 -element circuit. By orthogonality with $C_{1}^{*}, C_{2}^{*}$, 
$C_{3}^{*}, C_{4}^{*}$, and $C_{5}^{*}$, this circuit is either $\left\{d_{0}, f, c_{1}, c_{2}\right\}$ or $\left\{d_{0}, f, x, e\right\}$. But in the former case, $C_{0} \cup f$ is a 5 -element plane that intersects $C_{1}^{*}$ in two elements; a contradiction. So $\left\{d_{0}, f, x, e\right\}$ is a circuit. Hence $r\left(Y \cup\left\{g_{1}, g_{2}\right\}\right) \leq$ 5. We now claim that $M \backslash g_{1} \backslash g_{2}$ has an $N$-minor. As $\{x, e\}$ is a parallel pair in the matroid $M \backslash g_{1} \backslash d_{2} / d_{0} / f$, which has an $N$-minor, the matroid $M \backslash x \backslash g_{1} \backslash d_{2} / f$ also has an $N$-minor, and it follows that $M \backslash g_{1} / c_{2} / f$ has an $N$-minor, and finally $M \backslash g_{1} \backslash g_{2}$ also has an $N$-minor as claimed. Now $\left\{g_{1}, g_{2}\right\}$ is contained in a 4-element cocircuit $C_{8}^{*}$. By orthogonality, $C_{8}^{*} \subseteq Y \cup\left\{g_{1}, g_{2}\right\}$, so $r^{*}\left(Y \cup\left\{g_{1}, g_{2}\right\}\right) \leq 5$. Thus $\lambda\left(Y \cup\left\{g_{1}, g_{2}\right\}\right)=0$, in which case $|E(M)|=10$; a contradiction.

Now suppose $C_{5}^{*}=\left\{d_{0}, g_{1}, c_{1}, h_{1}\right\}$ for $h_{1} \in E(M)-\left(Y \cup\left\{g_{1}, g_{2}\right\}\right)$. Recall that there is a 4-element cocircuit $C_{6}^{*}$ containing $\left\{d_{0}, g_{2}\right\}$. By orthogonality with $C_{2}$ this cocircuit meets $\left\{d_{1}, x, c_{2}\right\}$, but cannot contain $d_{1}$, by orthogonality with $C_{0}, C_{1}$, and $C_{3}$, and cannot contain $x$, by orthogonality with $C_{0}, C_{1}$, and $C_{4}$. So, by orthogonality with $C_{3}$, either $C_{6}^{*}=\left\{d_{0}, g_{2}, c_{2}, e\right\}$ or $C_{6}^{*}=\left\{d_{0}, g_{2}, c_{2}, h_{2}\right\}$ for $h_{2} \in E(M)-\left(Y \cup\left\{g_{1}, g_{2}\right\}\right)$.

Next we claim that there is a cocircuit $C_{7}^{*}$ where either $C_{7}^{*}=\left\{d_{1}, g_{1}, x, p\right\}$ for $p \in E(M)-\left(Y \cup\left\{g_{1}, g_{2}\right\}\right)$, or $C_{7}^{*}=\left\{d_{1}, g_{1}, g_{2}, d_{2}\right\}$ in which case one of $\left\{x, f, c_{1}, c_{2}\right\},\left\{x, f, d_{1}, d_{2}\right\}$, or $\left\{x, f, g_{1}, g_{2}\right\}$ is also a cocircuit. Since $M \backslash d_{1} / c_{1} / x$ has an $N$-minor, and $\left\{d_{2}, g_{1}\right\}$ is a parallel pair in this matroid, $M \backslash d_{1} \backslash g_{1}$ has an $N$-minor. So there is a 4-element cocircuit $C_{7}^{*}$ containing $\left\{d_{1}, g_{1}\right\}$. By orthogonality with $C_{2}$, the cocircuit $C_{7}^{*}$ meets $\left\{x, c_{2}, g_{2}\right\}$. But $c_{2} \notin C_{7}^{*}$, by orthogonality with $C_{0}$ and $C_{4}$. If $g_{2} \in C_{7}^{*}$, then by orthogonality with $C_{1}$ and $C_{4}$, we have $C_{7}^{*}=\left\{d_{1}, g_{1}, g_{2}, d_{2}\right\}$; otherwise, $x \in C_{7}^{*}$, and, by orthogonality with $C_{0}$ and $C_{4}$, we have $C_{7}^{*}=\left\{d_{1}, g_{1}, x, p\right\}$ for $p \in E(M)-\left(Y \cup\left\{g_{1}, g_{2}\right\}\right)$. Now, in the case that $C_{7}^{*}=\left\{d_{1}, g_{1}, g_{2}, d_{2}\right\}$, the matroid $M \backslash d_{1} \backslash d_{2} / c_{1} / c_{2}$ has an $N$-minor and $\left\{g_{1}, g_{2}\right\}$ is a series pair, so $\left\{c_{2}, g_{1}\right\}$ and $\left\{c_{1}, g_{2}\right\}$ are $N$-contractible in $M$. Since $M / c_{1} / g_{1}$ has an $N$ minor and $\left\{x, d_{2}\right\}$ and $\left\{d_{1}, f\right\}$ are parallel pairs in this matroid (due to $C_{1}$ and $C_{3}$ ), the pair $\{x, f\}$ is $N$-deletable. So $\{x, f\}$ is contained in a 4-element cocircuit $C_{8}^{*}$. By orthogonality with $C_{3}$ and $C_{4}$, the cocircuit $C_{8}^{*}$ meets the disjoint sets $\left\{c_{1}, d_{1}, g_{1}\right\}$ and $\left\{c_{2}, d_{2}, g_{2}\right\}$. Again by orthogonality, this time with $C_{0}, C_{1}$, and $C_{2}$, the cocircuit $C_{8}^{*}$ is one of $\left\{x, f, c_{1}, c_{2}\right\},\left\{x, f, d_{1}, d_{2}\right\}$, or $\left\{x, f, g_{1}, g_{2}\right\}$.

Now we claim that there is a 4 -element circuit $C_{5}$ containing $\left\{h_{1}, x, d_{0}\right\}$ and one of $g_{1}, g_{2}$, or some $g^{\prime} \in E(M)-\left(Y \cup\left\{g_{1}, g_{2}, h_{1}\right\}\right)$. Since $M \backslash d_{0} / c_{1} / x$ has an $N$-minor, and $\left\{d_{2}, g_{1}\right\}$ is a parallel pair in this matroid, $M \backslash d_{0} \backslash g_{1} / x$ has an $N$-minor. As $\left\{h_{1}, c_{1}\right\}$ is a series pair in the latter matroid, $M / h_{1} / x$ has an $N$-minor. So $\left\{h_{1}, x\right\}$ is contained in a 4 -element circuit $C_{5}$. By orthogonality with $C_{1}^{*}$, the circuit $C_{5}$ meets $\left\{d_{1}, c_{1}, d_{0}\right\}$. But, by orthogonality with $C_{3}^{*}$ and $C_{5}^{*}$, we have $d_{1} \notin C_{5}$. If $c_{1} \in C_{5}$, then, by orthogonality with $C_{2}^{*}$ and $C_{4}^{*}$, we have $C_{5}=\left\{h_{1}, x, c_{1}, d_{2}\right\}$, but then $C_{1} \cup h_{1}$ is a 5-element plane that intersects $C_{1}^{*}$ in two elements; a contradiction. So $d_{0} \in C_{5}$. By orthogonality, $C_{5}$ does not meet $C_{3}^{*}$ or $C_{4}^{*}$, so the final element of $C_{5}$ is either $g_{1}, g_{2}$, or some $g^{\prime} \in E(M)-\left(Y \cup\left\{g_{1}, g_{2}, h_{1}\right\}\right)$. 
Suppose $C_{5}=\left\{h_{1}, x, d_{0}, g_{1}\right\}$. Then, by orthogonality, $C_{6}^{*}=$ $\left\{d_{0}, g_{2}, c_{2}, h_{1}\right\}$. First let $C_{7}^{*}=\left\{d_{1}, g_{1}, g_{2}, d_{2}\right\}$, so there is a circuit $C_{8}^{*}$ containing $\{x, f\}$. Then $C_{8}^{*}=\left\{x, f, g_{1}, g_{2}\right\}$, by orthogonality with $C_{5}$. Since $M \backslash d_{0} \backslash g_{2} / x$ has an $N$-minor and $\left\{c_{2}, h_{1}\right\}$ is a series pair in this matroid, $M \backslash g_{2} / x / h_{1}$ has an $N$-minor. Due to the circuit $C_{5}$, it follows that $M \backslash g_{1} \backslash g_{2} / h_{1}$ has an $N$-minor. Now, due to the cocircuit $C_{8}^{*}$, the pair $\left\{f, h_{1}\right\}$ is $N$-contractible. So there is a 4 -element circuit $C_{6}$ containing $\left\{f, h_{1}\right\}$. By orthogonality with $C_{3}^{*}, C_{4}^{*}, C_{5}^{*}$, and $C_{6}^{*}$, this circuit meets each of the sets $\left\{c_{1}, e, d_{2}\right\},\left\{c_{2}, e, d_{1}\right\},\left\{c_{1}, d_{0}, g_{1}\right\}$, and $\left\{c_{2}, d_{0}, g_{2}\right\}$. Thus either $C_{6}=\left\{f, h_{1}, c_{1}, c_{2}\right\}$ or $C_{6}=\left\{f, h_{1}, d_{0}, e\right\}$. But in either case $C_{6}$ intersects the cocircuit $C_{1}^{*}$ in a single element; a contradiction.

Now let $C_{7}^{*}=\left\{d_{1}, g_{1}, x, p\right\}$ for some $p \in E(M)-\left(Y \cup\left\{g_{1}, g_{2}\right\}\right)$. Since $M \backslash d_{0} \backslash d_{1} \backslash g_{1} / c_{1}$ has an $N$-minor, and $\{x, p\}$ is a series pair in this matroid, $\left\{c_{1}, p\right\}$ is $N$-contractible. So there is a 4 -element circuit containing $\left\{c_{1}, p\right\}$. There are two cases to consider, depending on whether or not $p=h_{1}$. In the case that $p=h_{1}$, the circuit contains $\left\{c_{1}, h_{1}\right\}$ and meets $\left\{d_{0}, x, d_{1}\right\}$ and $\left\{d_{2}, e, f\right\}$, by orthogonality with $C_{1}^{*}$ and $C_{3}^{*}$. By orthogonality with $C_{4}^{*}$, the circuit contains $d_{2}$; then, by orthogonality with $C_{2}^{*}$ and $C_{6}^{*}$, the final element is $d_{0}$. But then the circuit is $\left\{c_{1}, h_{1}, d_{2}, d_{0}\right\}$, and intersects $C_{7}^{*}$ in a single element; a contradiction. Now $p \neq h_{1}$, and the 4-element circuit containing $\left\{c_{1}, p\right\}$ meets $\left\{d_{1}, g_{1}, x\right\}$ and $\left\{d_{2}, e, f\right\}$ by orthogonality with $C_{7}^{*}$ and $C_{3}^{*}$. By orthogonality with $C_{4}^{*}$, the circuit contains $d_{2}$; then by orthogonality with $C_{1}^{*}$ and $C_{2}^{*}$, the circuit is $\left\{c_{1}, p, x, d_{2}\right\}$. But then $C_{1} \cup p$ is a 5-element plane that intersects $C_{1}^{*}$ in two elements; a contradiction.

Suppose $C_{5}=\left\{h_{1}, x, d_{0}, g_{2}\right\}$. Then, by orthogonality, $C_{7}^{*}=\left\{d_{1}, g_{1}, x, h_{1}\right\}$. Now, $M \backslash d_{0} \backslash d_{1} \backslash g_{1}$ has an $N$-minor, and $\left\{c_{1}, x, h_{1}\right\}$ is contained in a series class in this matroid. So, in particular, $M \backslash d_{1} / x / h_{1}$ has an $N$-minor. As $\left\{d_{0}, g_{2}\right\}$ is a parallel pair in this matroid, the pair $\left\{d_{1}, g_{2}\right\}$ is $N$-deletable in $M$. Thus there is a 4-element cocircuit $C_{9}^{*}$ containing $\left\{d_{1}, g_{2}\right\}$. By orthogonality with $C_{3}, C_{4}$, and $C_{5}$, this cocircuit meets $\left\{c_{1}, f, g_{1}\right\},\left\{c_{2}, d_{2}, f\right\}$, and $\left\{h_{1}, x, d_{0}\right\}$. Hence $f \in C_{9}^{*}$. By orthogonality with $C_{0}$ and $C_{1}$, we have $d_{0} \notin C_{9}^{*}$ and $x \notin C_{9}^{*}$, so $C_{9}^{*}=\left\{d_{1}, g_{2}, f, h_{1}\right\}$. Recall that $\left\{h_{1}, c_{1}\right\}$ is $N$ contractible. Thus there is a 4-element circuit $C_{7}$ containing $\left\{h_{1}, c_{1}\right\}$. By orthogonality with $C_{1}^{*}$ and $C_{9}^{*}$, this circuit meets $\left\{d_{1}, x, d_{0}\right\}$ and $\left\{d_{1}, g_{2}, f\right\}$. It follows that $C_{7}$ does not meet $C_{2}^{*}$, so $d_{1} \in C_{7}$. Then, by orthogonality with $C_{3}^{*}$, the final element in $C_{7}$ is either $e$ or $f$. But if $f \in C_{7}$, then $C_{3} \cup h_{1}$ is a 5-element plane that intersects the cocircuit $C_{1}^{*}$ in two elements; a contradiction. So $C_{7}=\left\{h_{1}, c_{1}, d_{1}, e\right\}$.

Now let $Y^{\prime}=C_{3} \cup C_{5}=C_{1}^{*} \cup C_{5}^{*} \cup C_{9}^{*}$, so $r^{*}\left(Y^{\prime}\right) \leq 5$ and $r\left(Y^{\prime}\right) \leq 6$. Then $d_{2}, c_{2}, e \in \operatorname{cl}\left(Y^{\prime}\right)$, due to $C_{1}, C_{2}$, and $C_{7}$ respectively, and $c_{2}, d_{2} \in \operatorname{cl}^{*}\left(Y^{\prime} \cup e\right)$, due to $C_{3}^{*}$ and $C_{4}^{*}$. Hence $r^{*}\left(Y^{\prime} \cup\left\{d_{2}, c_{2}, e\right\}\right) \leq 6$ and $r\left(Y^{\prime} \cup\left\{d_{2}, c_{2}, e\right\}\right) \leq$ 6 , so $\lambda\left(Y^{\prime} \cup\left\{d_{2}, c_{2}, e\right\}\right) \leq 1$, implying $|E(M)| \leq 12$. Recall that $C_{6}^{*}=$ $\left\{d_{0}, g_{2}, c_{2}, e\right\}$ or $C_{6}^{*}=\left\{d_{0}, g_{2}, c_{2}, h_{2}\right\}$ for $h_{2} \in E(M)-\left(Y \cup\left\{g_{1}, g_{2}\right\}\right)$. By orthogonality with $C_{7}$, we have $C_{6}^{*}=\left\{d_{0}, g_{2}, c_{2}, h_{2}\right\}$ and $h_{1} \neq h_{2}$. Now, as 
the elements $g_{1}, g_{2}, h_{1}, h_{2} \in E(M)-Y$ are distinct, $|E(M)|=12$. We work towards showing that $M$ is a nest of twisted cubes.

As $M \backslash d_{0} \backslash g_{2} / x$ has an $N$-minor, and $\left\{c_{2}, h_{2}\right\}$ is a series pair in this matroid, $\left\{h_{2}, x\right\}$ is $N$-contractible. So there is a 4-element circuit $C_{8}$ containing $\left\{h_{2}, x\right\}$. By orthogonality with $C_{6}^{*}$, this circuit contains an element in $\left\{d_{0}, g_{2}, c_{2}\right\}$. If $g_{2} \in C_{8}$, then, by orthogonality with $C_{1}^{*}$ and $C_{2}^{*}$, we have $C_{8}=\left\{h_{2}, x, g_{2}, d_{0}\right\}$, in which case $\left|C_{8} \cap C_{5}^{*}\right|=1$; a contradiction. If $c_{2} \in C_{8}$, then, by orthogonality with $C_{3}^{*}$ and $C_{4}^{*}$, we have $C_{8}=\left\{h_{2}, x, c_{2}, c_{1}\right\}$, in which case, again, $\left|C_{8} \cap C_{5}^{*}\right|=1$; a contradiction. So $d_{0} \in C_{8}$, in which case the final element is in $\left\{g_{1}, c_{1}, h_{1}\right\}$, by orthogonality with $C_{5}^{*}$. But the final element cannot be $c_{1}$, by orthogonality with $C_{3}^{*}$, and cannot be $h_{1}$, by orthogonality with $C_{9}^{*}$. So $C_{8}=\left\{h_{2}, x, d_{0}, g_{1}\right\}$.

We focus on uncovering the remaining circuits in the nest of twisted cubes. By circuit elimination on $C_{3}$ and $C_{7}$, there is a circuit contained in $\left\{c_{1}, e, f, g_{1}, h_{1}\right\}$. By orthogonality with $C_{1}^{*}$, we have that $C_{6}=\left\{e, f, g_{1}, h_{1}\right\}$ is a circuit. By circuit elimination on $C_{5}$ and $C_{8}$, the set $\left\{x, g_{1}, g_{2}, h_{1}, h_{2}\right\}$ contains a circuit, which is $C_{9}=\left\{g_{1}, g_{2}, h_{1}, h_{2}\right\}$, by orthogonality with $C_{1}^{*}$. Similarly, the set $\left\{c_{1}, f, x, d_{1}, d_{2}\right\}$ contains a circuit, by circuit elimination on $C_{1}$ and $C_{3}$, so $C_{10}=\left\{f, x, d_{1}, d_{2}\right\}$ is a circuit by orthogonality with $C_{5}^{*}$. By circuit elimination on $C_{6}$ and $C_{9}$, there is a circuit contained in $\left\{g_{1}, e, f, g_{2}, h_{2}\right\}$, which is $C_{11}=\left\{e, f, g_{2}, h_{2}\right\}$ by orthogonality with $C_{5}^{*}$. Now, by circuit elimination on $C_{4}$ and $C_{11}$, there is a circuit contained in $\left\{f, c_{2}, d_{2}, e, h_{2}\right\}$; by orthogonality with $C_{9}^{*}$, the circuit is $C_{12}=\left\{c_{2}, d_{2}, e, h_{2}\right\}$. The set $\left\{x, c_{1}, d_{0}, d_{2}, h_{2}\right\}$ also contains a circuit, by circuit elimination on $C_{1}$ and $C_{8}$; by orthogonality with $C_{7}^{*}$, the circuit is $C_{13}=\left\{c_{1}, d_{0}, d_{2}, h_{2}\right\}$. Finally, by circuit on elimination on $C_{0}$ and $C_{7}$, and orthogonality with $C_{3}^{*}$, we have that $C_{14}=\left\{c_{2}, d_{0}, d_{1}, h_{1}\right\}$ is a circuit.

We now turn to the remaining cocircuits. By cocircuit elimination on $C_{3}^{*}$ and $C_{4}^{*}$, there is a cocircuit contained in $\left\{e, c_{1}, c_{2}, d_{1}, d_{2}\right\}$, which, by orthogonality with $C_{6}$, is $C_{0}^{*}=\left\{c_{1}, c_{2}, d_{1}, d_{2}\right\}$. By cocircuit elimination on $C_{7}^{*}$ and $C_{9}^{*}$, there is a cocircuit contained in $\left\{d_{1}, x, f, g_{1}, g_{2}\right\}$; by orthogonality with $C_{7}$, this cocircuit is $C_{8}^{*}=\left\{x, f, g_{1}, g_{2}\right\}$. By cocircuit elimination on $C_{2}^{*}$ and $C_{6}^{*}$, there is a cocircuit contained in $\left\{d_{0}, d_{2}, x, g_{2}, h_{2}\right\}$; by orthogonality with $C_{0}$, this cocircuit is $C_{10}^{*}=\left\{d_{2}, x, g_{2}, h_{2}\right\}$. By cocircuit elimination on $C_{7}^{*}$ and $C_{10}^{*}$, there is a cocircuit contained in $\left\{x, d_{2}, f, g_{1}, h_{2}\right\}$; by orthogonality with $C_{2}$, this cocircuit is $C_{11}^{*}=\left\{d_{2}, f, g_{1}, h_{2}\right\}$. By cocircuit elimination on $C_{3}^{*}$ and $C_{11}^{*}$, there is a cocircuit contained in $\left\{f, c_{1}, e, g_{1}, h_{2}\right\}$; by orthogonality with $C_{4}$, this cocircuit is $C_{12}^{*}=\left\{c_{1}, e, g_{1}, h_{2}\right\}$. By cocircuit elimination on $C_{4}^{*}$ and $C_{9}^{*}$, there is a cocircuit contained in $\left\{f, c_{2}, e, g_{2}, h_{1}\right\}$; by orthogonality with $C_{3}$, the cocircuit is $C_{13}^{*}=\left\{c_{2}, e, g_{2}, h_{1}\right\}$. Finally, by cocircuit elimination on $C_{6}^{*}$ and $C_{13}^{*}$, the set $\left\{c_{2}, d_{0}, e, h_{1}, h_{2}\right\}$ contains a cocircuit; by orthogonality with $C_{2}$, the cocircuit is $C_{14}^{*}=\left\{d_{0}, e, h_{1}, h_{2}\right\}$. Now, using the labelling $\left(e_{1}, e_{2}, e_{3}, e_{4}, e_{5}, e_{6}, e_{1}^{\prime}, e_{2}^{\prime}, e_{3}^{\prime}, e_{4}^{\prime}, e_{5}^{\prime}, e_{6}^{\prime}\right)=$ $\left(d_{1}, d_{0}, g_{1}, d_{2}, e, g_{2}, h_{2}, f, c_{2}, h_{1}, x, c_{1}\right), M$ is a nest of twisted cubes. So (iii) holds. 
Now suppose $C_{5}=\left\{h_{1}, x, d_{0}, g^{\prime}\right\}$ for $g^{\prime} \in E(M)-\left(Y \cup\left\{g_{1}, g_{2}, h_{1}\right\}\right)$. Note that either $C_{7}^{*}=\left\{d_{1}, g_{1}, x, p\right\}$ for $p \in\left\{h_{1}, g^{\prime}\right\}$, or $C_{7}^{*}=\left\{d_{1}, g_{1}, g_{2}, d_{2}\right\}$ in which case there is another cocircuit $C_{8}^{*}$ containing $\{x, f\}$. In the latter case, the cocircuit $C_{8}^{*}$ is one of $\left\{x, f, c_{1}, c_{2}\right\},\left\{x, f, d_{1}, d_{2}\right\}$, or $\left\{x, f, g_{1}, g_{2}\right\}$. But then $C_{8}^{*}$ intersects $C_{5}$ in a single element $x$; a contradiction. So $C_{7}^{*}=$ $\left\{d_{1}, g_{1}, x, p\right\}$ for $p \in\left\{h_{1}, g^{\prime}\right\}$. Suppose that $p=g^{\prime}$, so $C_{7}^{*}=\left\{d_{1}, g_{1}, x, g^{\prime}\right\}$. Since $M \backslash d_{1} / c_{1} / x$ has an $N$-minor, $M \backslash d_{1} \backslash g_{1} / c_{1}$ also has an $N$-minor (due to $C_{1}$ ), and hence, due to $C_{7}^{*}$, the pair $\left\{c_{1}, g^{\prime}\right\}$ is $N$-contractible in $M$. Consider the 4-element circuit containing $\left\{c_{1}, g^{\prime}\right\}$. By orthogonality with $C_{1}^{*}$ and $C_{3}^{*}$, this circuit meets the disjoint sets $\left\{x, d_{0}, d_{1}\right\}$ and $\left\{e, f, d_{2}\right\}$. Due to $C_{5}^{*}$, the circuit contains $d_{0}$, and then $d_{2}$, due to $C_{2}^{*}$. But then it meets $C_{7}^{*}$ in a single element; a contradiction.

Now suppose $p=h_{1}$, so $C_{7}^{*}=\left\{d_{1}, g_{1}, x, h_{1}\right\}$. Since $M \backslash d_{0} \backslash d_{1} \backslash g_{1} / x$ has an $N$-minor, and $\left\{h_{1}, c_{1}\right\}$ is a series pair in this matroid, $M \backslash d_{1} / x / h_{1}$ has an $N$-minor. Due to $C_{5}$, it follows that $\left\{g^{\prime}, d_{1}\right\}$ is $N$-deletable in $M$. So there is a 4-element cocircuit $C_{9}^{*}$ containing $\left\{g^{\prime}, d_{1}\right\}$. By orthogonality with $C_{3}$ and $C_{5}$, this cocircuit meets the disjoint sets $\left\{c_{1}, f, g_{1}\right\}$ and $\left\{x, d_{0}, h_{1}\right\}$. Then, by orthogonality with $C_{2}$, we have $x \in C_{9}^{*}$. Again by orthogonality, this time with $C_{1}=\left\{g_{1}, c_{1}, x, d_{2}\right\}$, we see that either $C_{9}^{*}=\left\{g^{\prime}, d_{1}, x, g_{1}\right\}$ or $C_{9}^{*}=$ $\left\{g^{\prime}, d_{1}, x, c_{1}\right\}$. But in the first case, $\left\{g^{\prime}, d_{1}, x, g_{1}, h_{1}\right\}$ is a 5-element coplane, while in the second case, $C_{1}^{*} \cup g^{\prime}$ is a 5-element coplane; in either case, the 5 -element coplane intersects the circuit $C_{1}$ in two elements, contradicting orthogonality.

Finally we assume $g=g_{1}=g_{3}$ where $g, g_{2}$, and $g_{4}$ are distinct. Recall that $C_{5}^{*}=\left\{d_{0}, g, c_{1}, h\right\}$ where either $h=e$ or $h \in E(M)-\left(Y \cup\left\{g, g_{2}, g_{4}\right\}\right)$. Also, either $\left\{d_{0}, g_{2}, c_{2}\right\} \subseteq C_{6}^{*}$ or $C_{6}^{*}=\left\{d_{0}, g_{2}, e, d_{2}\right\}$. By orthogonality between $C_{6}^{*}$ and $C_{2}$, the latter is not possible; it follows, by orthogonality with $C_{1}, C_{3}$, and $C_{4}$, that $C_{6}^{*}=\left\{d_{0}, g_{2}, c_{2}, g_{4}\right\}$. Now, as $M \backslash d_{0} \backslash g_{2} / x$ has an $N$-minor, and $\left\{c_{2}, g_{4}\right\}$ is a series pair in this matroid, the pair $\left\{x, g_{4}\right\}$ is $N$-contractible. Thus there is a 4 -element circuit $C_{5}$ containing $\left\{x, g_{4}\right\}$. If $d_{0} \notin C_{5}$, then by orthogonality with $C_{6}^{*}$ and $C_{1}^{*}$, the circuit $C_{5}$ meets $\left\{c_{2}, g_{2}\right\}$ and $\left\{c_{1}, d_{1}\right\}$. In this case, by orthogonality with $C_{3}^{*}$ and $C_{4}^{*}$, we have $C_{5}=\left\{x, g_{4}, c_{2}, d_{1}\right\}$, but then $C_{2} \cup g_{4}$ is a 5 -element plane intersecting $C_{1}^{*}$ in two elements; a contradiction. So $d_{0} \in C_{5}$, and thus, by orthogonality with $C_{5}^{*}$ and $C_{3}^{*}$, either $C_{5}=\left\{x, g_{4}, d_{0}, g\right\}$ or $C_{5}=\left\{x, g_{4}, d_{0}, h\right\}$ and $h \neq e$.

Consider the latter case, where $C_{5}=\left\{x, g_{4}, d_{0}, h\right\}$ for $h \in E(M)-(Y \cup$ $\left.\left\{g, g_{2}, g_{4}\right\}\right)$. Then, as $M \backslash g_{2} / x / g_{4}$ has an $N$-minor and $\left\{d_{0}, h\right\}$ is a parallel pair in this matroid, the pair $\left\{h, g_{2}\right\}$ is $N$-deletable. So there is a 4-element cocircuit containing $\left\{h, g_{2}\right\}$. By orthogonality with $C_{5}$ this cocircuit meets $\left\{x, g_{4}, d_{0}\right\}$. But if the cocircuit contains $d_{0}$, then by orthogonality with $C_{0}$ it also meets $\left\{c_{1}, c_{2}, e\right\}$, in which case it intersects $C_{3}^{*}$ or $C_{4}^{*}$ in a single element; a contradiction. If the cocircuit contains $g_{4}$, then, by orthogonality with $C_{2}$ and $C_{4}$, it contains $c_{2}$, and thus intersects $C_{0}$ in a single element; a contradiction. So the cocircuit contains $\left\{h, g_{2}, x\right\}$, in which case, by orthogonality 
with $C_{1}$, the final element is in $\left\{g, c_{1}, d_{2}\right\}$, but then it intersects $C_{3}$ or $C_{4}$ in a single element; a contradiction.

So we may now assume that $C_{5}=\left\{x, g_{4}, d_{0}, g\right\}$. Then, as $M \backslash g_{2} / x / g_{4}$ has an $N$-minor and $\left\{d_{0}, g\right\}$ is a parallel pair in this matroid, the pair $\left\{g, g_{2}\right\}$ is $N$-deletable. So there is a 4 -element cocircuit containing $\left\{g, g_{2}\right\}$. By orthogonality with $C_{1}, C_{2}$, and $C_{3}$, the cocircuit meets $\left\{c_{1}, x, d_{2}\right\},\left\{c_{2}, x, d_{1}\right\}$, and $\left\{c_{1}, f, d_{1}\right\}$. Thus, if the cocircuit contains $d_{2}$, then it is $\left\{g, g_{2}, d_{2}, d_{1}\right\}$, but then it intersects $C_{4}$ in a single element; a contradiction. If it contains $c_{1}$, then, by orthogonality with $C_{0}$, it also contains $c_{2}$, and we again obtain the contradiction that the cocircuit intersects $C_{4}$ in a single element. So it contains $x$ and meets $\left\{c_{1}, f, d_{1}\right\}$. By orthogonality with $C_{0}$ and $C_{4}$, the cocircuit is $\left\{g, g_{2}, x, d_{1}\right\}$. By circuit elimination on $C_{1}^{*}$ and $C_{5}^{*}$, there is also a cocircuit contained in $\left\{c_{1}, g, h, x, d_{1}\right\}$; by orthogonality with $C_{0}$, this cocircuit is $\left\{g, h, x, d_{1}\right\}$. But then $\left\{g, g_{2}, h, x, d_{1}\right\}$ is a 5 -element coplane that intersects $C_{1}$ in two elements; a contradiction.

4.4.7. Suppose $\left|C_{1}^{*} \cap C_{2}^{*}\right|=2$ and $d_{1} \notin C_{2}^{*}$. Then the lemma holds.

Subproof. Let $X=C_{1}^{*} \cup C_{2}^{*}$. If $X$ is 3-separating, then (i) holds by 4.4.3, So we may assume that $X$ is not 3 -separating. Since $r^{*}(X)=4$, by 4.4 .2 , we have $r(X) \in\{5,6\}$. Let $C_{1}^{*}=\left\{d_{0}, d_{1}, c_{1}, x\right\}$ and $C_{2}^{*}=\left\{d_{0}, d_{2}, c_{2}, x\right\}$ for distinct $c_{1}, c_{2}, x \in E(M)-\left\{d_{0}, d_{1}, d_{2}\right\}$. Since $M \backslash d_{0} \backslash d_{1} \backslash d_{2}$ has an $N$-minor, and $\left\{x, c_{1}, c_{2}\right\}$ is contained in a series class in this matroid, the pair $\left\{c_{1}, c_{2}\right\}$ is $N$-contractible. Hence, this pair is contained in a 4-element circuit $C_{0}$. By orthogonality, $\left|C_{0} \cap X\right| \geq 3$. If $C_{0} \subseteq X$, then the lemma holds by 4.4.5.

So we may assume that $C_{0} \nsubseteq X$. By orthogonality, $C_{0}=\left\{c_{1}, c_{2}, x^{\prime}, e\right\}$ for some $x^{\prime} \in C_{1}^{*} \cap C_{2}^{*}$ and $e \in E(M)-X$. If $x^{\prime}=x$, then, as $M \backslash d_{0} \backslash d_{1} \backslash d_{2} / c_{1} / c_{2}$ has an $N$-minor, and $\{x, e\}$ is a parallel pair in this matroid, $M \backslash x \backslash d_{1} \backslash d_{2}$ has an $N$-minor. So we may assume, up to swapping the labels on $x$ and $d_{0}$, that $x^{\prime}=d_{0}$; that is, $C_{0}=\left\{c_{1}, c_{2}, d_{0}, e\right\}$. Moreover, if $e \in \mathrm{cl}^{*}(X)$, then the coindependent circuit $C_{0}$ cospans $X \cup e$, and $\left\{d_{1}, d_{2}\right\} \subseteq \operatorname{cl}^{*}\left(C_{0}\right)-C_{0}$, so (ii) holds by 4.4.1, So $e \notin \mathrm{cl}^{*}(X)$.

As $M \backslash d_{1} / c_{1} / c_{2}$ has an $N$-minor, and $\left\{d_{0}, e\right\}$ is a parallel pair in this matroid, $\left\{d_{1}, e\right\}$ is $N$-deletable. Let $C_{3}^{*}$ be the 4-element cocircuit containing $\left\{d_{1}, e\right\}$. Then $C_{3}^{*}$ meets $\left\{c_{1}, d_{0}, c_{2}\right\}$, by orthogonality with $C_{0}$. Since $e \notin$ $\mathrm{cl}^{*}(X)$, the cocircuit $C_{3}^{*}$ also contains an element $f_{1} \in E(M)-(X \cup e)$. Similarly, we let $C_{4}^{*}$ be the cocircuit containing $\left\{d_{2}, e\right\}$, and observe that $C_{4}^{*}$ contains an element $f_{2} \in E(M)-(X \cup e)$.

Suppose that $C_{3}^{*}=\left\{d_{1}, e, c_{1}, f_{1}\right\}$. Let $C_{1}$ be the circuit containing $\left\{c_{1}, x\right\}$. By orthogonality with $C_{3}^{*}$ and $C_{2}^{*}$, the circuit $C_{1}$ meets $\left\{d_{1}, e, f_{1}\right\}$ and $\left\{c_{2}, d_{2}, d_{0}\right\}$. We claim that $e \notin C_{1}$. Towards a contradiction, suppose $e \in C_{1}$. Then $C_{1}$ does not meet $\left\{c_{2}, d_{0}\right\}$, as otherwise $r\left(C_{0} \cup x\right)=3$, in which case $C_{0} \cup x$ is a 5-element plane that intersects the cocircuit $C_{3}^{*}$ in two elements, contradicting orthogonality. So $C_{1}=\left\{c_{1}, x, e, d_{2}\right\}$. But in this case, by circuit elimination with $C_{0}$, the set $\left\{c_{1}, x, d_{0}, c_{2}, d_{2}\right\}$ contains a circuit. This 
circuit cannot contain $c_{1}$, by orthogonality with $C_{3}^{*}$, so $\left\{x, d_{0}, c_{2}, d_{2}\right\}=C_{2}^{*}$ is a quad; a contradiction.

Now $C_{1}$ meets $\left\{d_{1}, f_{1}\right\}$ and $\left\{c_{2}, d_{2}, d_{0}\right\}$. Suppose $d_{1} \in C_{1}$. If $C_{1}=$ $\left\{c_{1}, x, d_{1}, c_{2}\right\}$, then $\left\{d_{0}, d_{2}\right\} \subseteq \operatorname{cl}^{*}\left(C_{1}\right)-C_{1}$, so (ii) holds by 4.4.1. If $C_{1}=$ $\left\{c_{1}, x, d_{1}, d_{0}\right\}$, then $C_{1}$ is a quad; a contradiction. So $C_{1}=\left\{c_{1}, x, d_{1}, d_{2}\right\}$. Recall the cocircuit $C_{4}^{*}$ containing $\left\{d_{2}, e\right\}$ and an element $f_{2} \in E(M)-(X \cup e)$. By orthogonality with $C_{0}$ and $C_{1}$, this cocircuit meets $\left\{c_{1}, d_{0}, c_{2}\right\}$ and $\left\{c_{1}, x, d_{1}\right\}$. So $C_{4}^{*}=\left\{d_{2}, e, c_{1}, f_{2}\right\}$.

By cocircuit elimination on $C_{3}^{*}$ and $C_{4}^{*}$, the set $\left\{d_{1}, d_{2}, e, f_{1}, f_{2}\right\}$ contains a cocircuit. But this set intersects $C_{0}$ in a single element, $e$, so $C_{0}^{*}=$ $\left\{d_{1}, d_{2}, f_{1}, f_{2}\right\}$ is a cocircuit, and, in particular, $f_{1} \neq f_{2}$.

We work towards a contradiction by showing $\left\{f_{1}, f_{2}\right\} \subseteq \operatorname{cl}(X \cup e)$. Since $M \backslash d_{0} \backslash d_{1} \backslash d_{2}$ has an $N$-minor, and $\left\{f_{1}, f_{2}\right\}$ and $\left\{c_{2}, x\right\}$ are series pairs in this matroid, $\left\{f_{1}, c_{2}\right\}$ and $\left\{f_{2}, x\right\}$ are $N$-contractible. First, consider the 4element circuit containing $\left\{f_{1}, c_{2}\right\}$. By orthogonality, it meets $\left\{d_{1}, e, c_{1}\right\}$ and $\left\{x, d_{0}, d_{2}\right\}$. So $f_{1} \in \operatorname{cl}(X \cup e)$. Now consider the 4-element circuit containing $\left\{f_{2}, x\right\}$. By orthogonality with $C_{1}^{*}$ and $C_{2}^{*}$, this circuit meets $\left\{c_{1}, d_{1}, d_{0}\right\}$ and $\left\{c_{2}, d_{2}, d_{0}\right\}$. Suppose this circuit contains $d_{0}$. By orthogonality with $C_{0}^{*}$ and $C_{4}^{*}$, the circuit also meets $\left\{d_{1}, d_{2}, f_{1}\right\}$ and $\left\{d_{2}, e, c_{1}\right\}$, so in this case the circuit is $\left\{f_{2}, x, d_{0}, d_{2}\right\}$. Thus $f_{2} \in \operatorname{cl}(X)$ in either case, and $r\left(X \cup\left\{e, f_{1}, f_{2}\right\}\right)=$ $r(X)=5$. But $\left\{c_{1}, d_{0}, d_{1}, d_{2}, f_{1}\right\}$ (for example) cospans $X \cup\left\{e, f_{1}, f_{2}\right\}$, so $r^{*}\left(X \cup\left\{e, f_{1}, f_{2}\right\}\right)=5$, implying $\lambda\left(X \cup\left\{e, f_{1}, f_{2}\right\}\right)=1$. Thus $|E(M)|=10$; a contradiction.

We deduce that $d_{1} \notin C_{1}$, so $C_{1}=\left\{c_{1}, x, p, f_{1}\right\}$ for some $p \in\left\{c_{2}, d_{2}, d_{0}\right\}$. Recall the cocircuit $C_{4}^{*}$ that contains $\left\{d_{2}, e\right\}$ and an element $f_{2} \in E(M)-$ $(X \cup e)$. By orthogonality with $C_{0}$, we see $C_{4}^{*}=\left\{d_{2}, e, q, f_{2}\right\}$ for some $q \in\left\{c_{1}, c_{2}, d_{0}\right\}$. By orthogonality between $C_{1}$ and $C_{4}^{*}$, either $(p, q) \in$ $\left\{\left(d_{2}, c_{1}\right),\left(c_{2}, d_{0}\right),\left(d_{0}, c_{2}\right)\right\}$ or $f_{1}=f_{2}$.

Before considering these subcases, suppose that $\left\{c_{1}, x, c_{2}, f_{1}\right\}$ is a circuit. Let $Y=C_{0} \cup\left\{x, f_{1}\right\}$, so $\left\{c_{1}, x, c_{2}, f_{1}\right\} \subseteq Y$. Observe that $r(Y) \leq 4$ and $r_{M \backslash d_{1}}^{*}(Y) \leq 4$. Hence $Y$ is 3 -separating in $M \backslash d_{1}$, and $d_{2} \in \mathrm{cl}_{M \backslash d_{1}}^{*}(Y)$. Towards an application of Lemma4.2, it remains to show that $|Y-(C \cup D)| \leq$ 2 for an $N$-labelling $(C, D)$ with $\left\{d_{1}, d_{2}\right\} \subseteq D$. To this end, note that $M \backslash d_{0} \backslash d_{1} \backslash d_{2} / c_{1} / x$ has an $N$-minor, and this matroid has the parallel pair $\left\{c_{2}, f_{1}\right\}$, so we may assume that $|Y-(C \cup D)| \leq 2$ as required. Now (ii) holds in this case, by Lemma 4.2. We may now assume that $\left\{c_{1}, x, c_{2}, f_{1}\right\}$ is not a circuit; in particular, $p \neq c_{2}$.

Suppose $q=c_{1}$, so $C_{4}^{*}=\left\{d_{2}, e, c_{1}, f_{2}\right\}$. By cocircuit elimination on $C_{3}^{*}$ and $C_{4}^{*}$, there is a cocircuit contained in $\left\{d_{1}, d_{2}, e, f_{1}, f_{2}\right\}$. But this set intersects $C_{0}$ in a single element, $e$, so $C_{0}^{*}=\left\{d_{1}, d_{2}, f_{1}, f_{2}\right\}$ is a cocircuit. In particular, when $q=c_{1}$, we have $f_{1} \neq f_{2}$.

We now consider cases. First, suppose $(p, q)=\left(d_{0}, c_{2}\right)$; we will show this leads to a contradiction. Recall that $C_{1}=\left\{c_{1}, x, d_{0}, f_{1}\right\}$ and $C_{4}^{*}=$ $\left\{d_{2}, e, c_{2}, f_{2}\right\}$, so $f_{1} \neq f_{2}$, by orthogonality. Observe that $\left\{e, f_{1}\right\} \subseteq \operatorname{cl}(X-$ $\left.d_{2}\right)$. As the pair $\left\{c_{2}, x\right\}$ is $N$-contractible, it is contained in a 4 -element 
circuit $C_{2}$ that, by orthogonality with $C_{1}^{*}$ and $C_{4}^{*}$, meets $\left\{c_{1}, d_{1}, d_{0}\right\}$ and $\left\{d_{2}, e, f_{2}\right\}$.

We claim that either $C_{2}=\left\{c_{2}, x, d_{0}, f_{2}\right\}$ or $C_{2}=\left\{c_{2}, x, d_{1}, e\right\}$. First, observe that if $c_{1} \in C_{2}$, then, by orthogonality with $C_{3}^{*}$, we have $C_{2}=$ $\left\{c_{2}, x, c_{1}, e\right\}$. But then $C_{0} \cup C_{2}$ is a 5-element plane that intersects the cocircuit $C_{4}^{*}$ in two elements; a contradiction. So $C_{2}$ meets $\left\{d_{1}, d_{0}\right\}$. If $d_{0} \in C_{2}$, then $C_{2}$ intersects $C_{3}^{*}$ in at most one element, so $C_{2} \cap C_{3}^{*}=\emptyset$. As $C_{2}$ meets $\left\{d_{2}, e, f_{2}\right\}$, and $C_{2} \neq C_{2}^{*}$, we deduce that $C_{2}=\left\{c_{2}, x, d_{0}, f_{2}\right\}$ when $d_{0} \in C_{2}$. Finally, if $d_{1} \in C_{2}$, then, by orthogonality with $C_{3}^{*}$, the final element is in $\left\{c_{1}, e, f_{1}\right\}$. As the only element of this set that is in $\left\{d_{2}, e, f_{2}\right\}$ is $e$, we have $C_{2}=\left\{c_{2}, x, d_{1}, e\right\}$. This proves the claim.

Next we claim that $d_{2} \in \operatorname{cl}\left(X-d_{2}\right)$, so $r(X)=5$. Observe that $M \backslash d_{1} / c_{1} / c_{2}$ has an $N$-minor, and $\left\{d_{0}, e\right\}$ is a parallel pair in this matroid, so $M \backslash e \backslash d_{1} / c_{2}$ also has an $N$-minor. As $\left\{c_{1}, f_{1}\right\}$ is a series pair in the latter matroid, $\left\{f_{1}, c_{2}\right\}$ is $N$-contractible. So there is a 4-element circuit $C_{3}$ containing $\left\{f_{1}, c_{2}\right\}$. By orthogonality with $C_{2}^{*}$ and $C_{3}^{*}$, this circuit contains an element in $\left\{x, d_{0}, d_{2}\right\}$, and an element in $\left\{d_{1}, c_{1}, e\right\}$. If $d_{2} \notin C_{3}$, then $C_{3}$ meets $C_{1}^{*}$, so by orthogonality $\left|C_{3} \cap C_{1}^{*}\right|=2$. But then $C_{3} \cap C_{4}^{*}=\left\{c_{2}\right\}$; a contradiction. So $d_{2} \in C_{3}$, in which case $C_{3}$ intersects $C_{1}^{*}$ in at most one element; thus $C_{3} \cap C_{1}^{*}=\emptyset$, by orthogonality. Hence $C_{3}=\left\{f_{1}, c_{2}, d_{2}, e\right\}$, so $d_{2} \in \operatorname{cl}\left(\left(X-d_{2}\right) \cup\left\{e, f_{1}\right\}\right)=\operatorname{cl}\left(X-d_{2}\right)$, as required.

Now, if $C_{2}=\left\{c_{2}, x, d_{0}, f_{2}\right\}$, then $\left\{d_{2}, e, f_{1}, f_{2}\right\} \subseteq \operatorname{cl}\left(X-d_{2}\right)$, so $r\left(X \cup\left\{f_{1}, f_{2}, e\right\}\right)=5$. As $r^{*}\left(X \cup\left\{f_{1}, f_{2}, e\right\}\right) \leq 5$, we have $\lambda\left(X \cup\left\{f_{1}, f_{2}, e\right\}\right) \leq$ 1 , so $|E(M)| \leq 10$; a contradiction. So we may assume that $C_{2}=$ $\left\{c_{2}, x, d_{1}, e\right\}$. By circuit elimination with $C_{0}$, there is a circuit contained in $\left\{c_{1}, c_{2}, x, d_{0}, d_{1}\right\}$. By orthogonality with $C_{4}^{*}$, this circuit does not contain $c_{2}$. Thus $C_{1}^{*}=\left\{c_{1}, x, d_{0}, d_{1}\right\}$ is a quad; a contradiction.

Next, suppose $(p, q)=\left(d_{2}, c_{1}\right)$; that is, $C_{1}=\left\{c_{1}, x, d_{2}, f_{1}\right\}$ and $C_{4}^{*}=$ $\left\{d_{2}, e, c_{1}, f_{2}\right\}$. Recall that when $q=c_{1}$, we have that $C_{0}^{*}=\left\{d_{1}, d_{2}, f_{1}, f_{2}\right\}$ is a cocircuit, and $f_{1} \neq f_{2}$. We work towards a contradiction by showing that $r\left(X \cup\left\{e, f_{1}, f_{2}\right\}\right)=5$. To start with, note that $\left\{e, f_{1}\right\} \subseteq \operatorname{cl}\left(X-d_{1}\right)$. As $\left\{x, c_{2}\right\}$ and $\left\{f_{1}, f_{2}\right\}$ are series pairs in $M \backslash d_{0} \backslash d_{1} \backslash d_{2}$, the pairs $\left\{x, f_{2}\right\}$ and $\left\{c_{2}, f_{1}\right\}$ are $N$-contractible. Let $C_{3}$ be the 4-element circuit containing $\left\{x, f_{2}\right\}$. This circuit meets $\left\{d_{0}, c_{1}, d_{1}\right\}$ by orthogonality. This circuit can only intersect $C_{3}^{*}=\left\{c_{1}, d_{1}, e, f_{1}\right\}$ in at most one element, so it does not meet $C_{3}^{*}$. Thus $d_{0} \in C_{3}$. By orthogonality with $C_{4}^{*}$, the final element is in $\left\{d_{2}, e, c_{1}\right\}$. But as $\left\{e, c_{1}\right\} \subseteq C_{3}^{*}$, we have $C_{3}=\left\{x, f_{2}, d_{0}, d_{2}\right\}$. In particular, $f_{2} \in \operatorname{cl}\left(X-d_{1}\right)$.

We claim that $d_{1} \in \operatorname{cl}\left(X-d_{1}\right)$. Let $C_{4}$ be the 4-element circuit containing $\left\{c_{2}, f_{1}\right\}$. By orthogonality with $C_{2}^{*}$ and $C_{3}^{*}$, the circuit $C_{4}$ meets the disjoint sets $\left\{d_{0}, x, d_{2}\right\}$ and $\left\{d_{1}, e, c_{1}\right\}$. Moreover, due to the cocircuit $C_{0}^{*}$, either $d_{1} \in C_{4}$ or $d_{2} \in C_{4}$. Suppose $d_{2} \in C_{4}$. Then $C_{4}$ intersects $C_{1}^{*}$ in at most one element, so $C_{4} \cap C_{1}^{*}=\emptyset$, implying $C_{4}=\left\{c_{2}, f_{1}, d_{2}, e\right\}$. By circuit elimination on $C_{1}$ and $C_{4}$, there is a circuit contained in $\left\{c_{1}, c_{2}, e, x, f_{1}\right\}$. But this set intersects $C_{0}^{*}$ in a single element, $f_{1}$, so $\left\{c_{1}, c_{2}, e, x\right\}$ is a circuit. Now 
$C_{0} \cup x$ is a 5-element plane that intersects the cocircuit $C_{3}^{*}$ in two elements, contradicting orthogonality. So $d_{1} \in C_{4}$. Thus $d_{1} \in \operatorname{cl}\left(Y-d_{1}\right)=\operatorname{cl}\left(X-d_{1}\right)$, as claimed. Finally, as $r\left(X \cup\left\{e, f_{1}, f_{2}\right\}\right)=5$, we see that $\lambda\left(X \cup\left\{e, f_{1}, f_{2}\right\}\right) \leq$ $5+5-9=1$, so $|E(M)| \leq 10$; a contradiction.

Now we assume that $f_{1}=f_{2}$. By orthogonality between $C_{1}$ and $C_{4}^{*}$, we have either $q=c_{1}, p=d_{2}$, or $p=q$ and $p \in\left\{c_{2}, d_{0}\right\}$. However, we have seen that if $q=c_{1}$, then $f_{1} \neq f_{2}$; so $q \neq c_{1}$.

Suppose $p=d_{2}$, so $C_{1}=\left\{c_{1}, x, d_{2}, f_{1}\right\}$, and recall that $C_{4}^{*}=\left\{d_{2}, e, q, f_{1}\right\}$ with $q \in\left\{c_{1}, c_{2}, d_{0}\right\}$. If $q=d_{0}$, then by cocircuit elimination on $C_{3}^{*}$ and $C_{4}^{*}$, there is a cocircuit contained in $\left\{d_{0}, d_{1}, d_{2}, c_{1}, e\right\}$. Then, as $e \notin \mathrm{cl}^{*}(X)$, the set $\left\{d_{0}, d_{1}, d_{2}, c_{1}\right\}$ is a cocircuit, implying $r^{*}(X)<4$; a contradiction. Since $q \neq c_{1}$, we have $C_{4}^{*}=\left\{d_{2}, e, c_{2}, f_{1}\right\}$. As the pair $\left\{c_{2}, x\right\}$ is $N$-contractible, it is contained in a 4-element circuit $C_{2}$ that, by orthogonality with $C_{1}^{*}$ and $C_{4}^{*}$, meets $\left\{c_{1}, d_{1}, d_{0}\right\}$ and $\left\{d_{2}, e, f_{1}\right\}$. By orthogonality with $C_{3}^{*}$, either $\left\{d_{0}, d_{2}\right\} \subseteq C_{2}$ or $\left\{d_{0}, d_{2}\right\} \cap C_{2}=\emptyset$. But in the former case $C_{2}=C_{2}^{*}$; a contradiction. Now, if $c_{1} \in C_{2}$, then either $C_{2}=\left\{c_{2}, x, c_{1}, e\right\}$ or $C_{2}=$ $\left\{c_{2}, x, c_{1}, f_{1}\right\}$, so $\left|C_{2} \cap C_{0}\right|=3$ or $\left|C_{2} \cap C_{1}\right|=3$. In either case, it follows that there is a 5-element plane intersecting a cocircuit in two elements; a contradiction. So either $C_{2}=\left\{c_{2}, x, d_{1}, e\right\}$ or $C_{2}=\left\{c_{2}, x, d_{1}, f_{1}\right\}$. In the former case, by circuit elimination with $C_{0}$, there is a circuit contained in $\left\{c_{1}, c_{2}, x, d_{0}, d_{1}\right\}$. By orthogonality with $C_{4}^{*}$, the set $\left\{c_{1}, x, d_{0}, d_{1}\right\}$ is a circuit, so $C_{1}^{*}$ is a quad; a contradiction. So $C_{2}=\left\{c_{2}, x, d_{1}, f_{1}\right\}$. Now $M \backslash d_{2} / c_{2} / x$ has an $N$-minor, and $\left\{d_{1}, f_{1}\right\}$ is a parallel pair in this matroid, so $M \backslash d_{2} \backslash f_{1} / x$ has an $N$-minor. The latter matroid has $\left\{c_{2}, e\right\}$ as a series pair, so $\{e, x\}$ is $N$-contractible. Thus $\{e, x\}$ is contained in a 4-element circuit $C_{3}$. By orthogonality with $C_{2}^{*}$ and $C_{3}^{*}$, the circuit $C_{3}$ meets $\left\{d_{0}, d_{2}, c_{2}\right\}$ and $\left\{d_{1}, c_{1}, f_{1}\right\}$. By orthogonality with $C_{1}^{*}$ and $C_{4}^{*}$, either $\left\{d_{0}, f_{1}\right\} \subseteq C_{3}$, or $\left\{d_{0}, f_{1}\right\} \cap C_{3}=\emptyset$. But if $C_{3}=\left\{e, x, d_{0}, f_{1}\right\}$, then $C_{3}=C_{1}^{*} \triangle C_{3}^{*}$. As $M \backslash d_{0} \backslash d_{1} \backslash f_{1} / c_{1} / x$ has an $N$-minor, the lemma holds, by 4.4.4, in this case. So we may assume that $C_{3}$ meets $\left\{c_{1}, d_{1}\right\}$ and $\left\{c_{2}, d_{2}\right\}$. If $c_{1} \in C_{3}$, then $C_{0} \cup$ $C_{3}$ or $C_{1} \cup C_{3}$ is a 5-element plane that intersects a cocircuit in two elements, contradicting orthogonality. Similarly if $C_{3}=\left\{e, x, d_{1}, c_{2}\right\}$, then $C_{3} \cup C_{2}$ is a 5 -element plane that intersects $C_{1}^{*}$ in two elements; a contradiction. So $C_{3}=$ $\left\{e, x, d_{1}, d_{2}\right\}$. By circuit elimination with $C_{2}$, there is a circuit contained in $\left\{c_{2}, d_{1}, d_{2}, e, f\right\}$. By orthogonality with $C_{1}^{*}$, we see that $C_{3}^{*}=\left\{c_{2}, d_{2}, e, f\right\}$ is a quad; a contradiction. This completes the subcase where $p=d_{2}$.

Suppose $p=q$ and $p \in\left\{c_{2}, d_{0}\right\}$. We have seen that $p \neq c_{2}$, so $p=q=d_{0}$. In particular, $C_{4}^{*}=\left\{d_{2}, e, d_{0}, f_{1}\right\}$. As the pair $\left\{c_{2}, x\right\}$ is $N$-contractible, it is contained in a 4 -element circuit $C_{2}$ that, by orthogonality with $C_{1}^{*}$, meets $\left\{c_{1}, d_{1}, d_{0}\right\}$. If $d_{0} \in C_{2}$, then, by orthogonality with $C_{4}^{*}$, the final element of $C_{2}$ is in $\left\{d_{2}, e, f_{1}\right\}$. But $\left\{c_{2}, x, d_{0}, d_{2}\right\}$ is not a cocircuit, for otherwise it would be a quad; and $e, f_{1} \notin \mathrm{cl}^{*}(X)$. So $d_{0} \notin C_{2}$. Again using that $e, f_{1} \notin \mathrm{cl}^{*}(X)$, it now follows that $C_{2}=\left\{c_{2}, x, c_{1}, d_{1}\right\}$. Thus $C_{2}$ is coindependent, and hence $\left\{d_{0}, d_{2}\right\} \subseteq \mathrm{cl}^{*}\left(C_{2}\right)-C_{2}$. So (ii) holds by 4.4.1. This completes the case where $C_{3}^{*}=\left\{d_{1}, e, c_{1}, f_{1}\right\}$. 
Now we may assume that $C_{3}^{*}=\left\{d_{1}, e, s_{1}, f_{1}\right\}$ for $s_{1} \in\left\{d_{0}, c_{2}\right\}$. By symmetry, $C_{4}^{*}=\left\{d_{2}, e, s_{2}, f_{2}\right\}$ for some $s_{2} \in\left\{d_{0}, c_{1}\right\}$ and $f_{2} \in E(M)-(X \cup e)$.

Suppose that $s_{1}=s_{2}=d_{0}$, so $C_{3}^{*}=\left\{d_{1}, e, d_{0}, f_{1}\right\}$ and $C_{4}^{*}=\left\{d_{2}, e, d_{0}, f_{2}\right\}$. Then, by cocircuit elimination, $\left\{d_{1}, d_{2}, e, f_{1}, f_{2}\right\}$ contains a cocircuit. But this set intersects the circuit $C_{0}$ in a single element, $e$, so $C_{0}^{*}=\left\{d_{1}, d_{2}, f_{1}, f_{2}\right\}$ is a cocircuit. In particular, $f_{1} \neq f_{2}$.

Let $Y=X \cup\left\{e, f_{1}, f_{2}\right\}$, and note that $r^{*}(Y) \leq 5$. We claim that $r(Y)=r(X)$. As $\left\{d_{0}, d_{1}\right\} \subseteq C_{3}^{*}$ and $\left\{d_{0}, d_{2}\right\} \subseteq C_{4}^{*}$, we have $r^{*}\left(C_{3}^{*} \cup C_{4}^{*}\right)=4$ by 4.4.2. Thus, in $M \backslash d_{0} \backslash d_{1} \backslash d_{2}$, both $\left\{c_{1}, x, c_{2}\right\}$ and $\left\{f_{1}, e, f_{2}\right\}$ are contained in series classes. So a matroid obtained by contracting a pair of elements in $\left\{c_{1}, x, c_{2}\right\}$, and a pair in $\left\{f_{1}, e, f_{2}\right\}$, from $M \backslash d_{0} \backslash d_{1} \backslash d_{2}$, has an $N$-minor. In particular, $\{c, f\}$ is $N$-contractible for distinct $c, f \in\left\{c_{1}, x, c_{2}, f_{1}, e, f_{2}\right\}$. We first consider the 4-element circuits $C_{3}$ and $C_{4}$ containing $\left\{c_{1}, f_{2}\right\}$ and $\left\{c_{2}, f_{1}\right\}$, respectively. If $d_{0}$ is in either of these circuits, then, by orthogonality, the circuit also meets the disjoint sets $C_{2}^{*}-d_{0}$ and $C_{3}^{*}-d_{0}$; a contradiction. So $C_{3}$ meets $\left\{x, d_{1}\right\}$ and $\left\{e, d_{2}\right\}$. By orthogonality with $C_{2}^{*}$, either $C_{3}=\left\{c_{1}, f_{2}, x, d_{2}\right\}$ or $C_{3}=\left\{c_{1}, f_{2}, d_{1}, e\right\}$. Similarly, $C_{4}=\left\{c_{2}, f_{1}, x, d_{1}\right\}$ or $C_{4}=\left\{c_{2}, f_{1}, d_{2}, e\right\}$. As $e \in \operatorname{cl}(X)$, we now also have $\left\{f_{1}, f_{2}\right\} \subseteq \operatorname{cl}(X \cup e)=$ $\operatorname{cl}(X)$, so $r(Y)=r(X)$.

Now, if $r(X)=5$, then, as $r^{*}(Y) \leq 5$ and $|Y|=9$, we have $\lambda(Y) \leq 1$, so $|E(M)| \leq 10$; a contradiction. So $r(X)=6$.

We will show that (ii) holds. Suppose that $C_{3}=\left\{c_{1}, f_{2}, d_{1}, e\right\}$. There is also a 4 -element circuit $C_{5}$ containing $\left\{f_{2}, x\right\}$. By orthogonality with $C_{4}^{*}$, this circuit meets $\left\{d_{2}, d_{0}, e\right\}$. If $d_{2} \in C_{5}$, then $C_{5}$ meets $C_{1}^{*}-x$ but is disjoint from $C_{3}^{*}$; so $C_{5}=\left\{f_{2}, x, d_{2}, c_{1}\right\}$. Now $\left\{e, f_{2}\right\} \subseteq \operatorname{cl}\left(X-d_{1}\right)$, so $d_{1} \in \operatorname{cl}\left(X-d_{1}\right)$ due to $C_{3}$. Thus $r(X)=5$; a contradiction. So $d_{2} \notin C_{5}$. Then $C_{5}$ meets $\left\{d_{0}, e\right\}$, and, by orthogonality with $C_{0}^{*}$ and $C_{3}^{*}$, the final element is in $\left\{d_{1}, f_{1}\right\}$. By orthogonality with $C_{2}^{*}$ it follows that $C_{5}=\left\{f_{2}, x, d_{0}, q\right\}$ for some $q \in\left\{d_{1}, f_{1}\right\}$. Now due to $C_{0}$ and $C_{3}$, we have $\left\{e, f_{2}\right\} \subseteq \operatorname{cl}\left(\left\{c_{1}, c_{2}, d_{0}, d_{1}\right\}\right)$, so if $q=d_{1}$ then $x \in \operatorname{cl}(X-x)$ and $r(X)=5$; a contradiction. So $C_{5}=\left\{f_{2}, x, d_{0}, f_{1}\right\}$. If $C_{4}=\left\{c_{2}, f_{1}, d_{2}, e\right\}$, then $\left\{f_{1}, f_{2}\right\} \subseteq \operatorname{cl}(X-x)$, so $x \in \operatorname{cl}(X-x)$ due to $C_{5}$, implying $r(X)=5$; a contradiction. So $C_{4}=\left\{c_{2}, f_{1}, x, d_{1}\right\}$. Let $X^{\prime}=C_{1}^{*} \cup C_{3}^{*}$, and observe that $r^{*}\left(X^{\prime}\right)=4$, by 4.4.2. Due to the circuits $C_{0}$ and $C_{4}$ contained in $X^{\prime} \cup c_{2}$, we have $r_{M / c_{2}}\left(X^{\prime}\right)=4$, so $\lambda_{M / c_{2}}\left(X^{\prime}\right)=2$, with $c_{2} \in \operatorname{cl}\left(X^{\prime}\right)$ and $f_{2} \in \operatorname{cl}_{M / c_{2}}\left(X^{\prime}\right)$. As $\left\{c_{2}, f_{2}\right\}$ is an $N$-contractible pair, and $M \backslash d_{0} \backslash d_{1} / c_{2} / f_{2} / x / e$ has an $N$-minor, (ii) holds by the dual of Lemma 4.2 .

Now $C_{3}=\left\{c_{1}, f_{2}, x, d_{2}\right\}$ and, by symmetry, $C_{4}=\left\{c_{2}, f_{1}, x, d_{1}\right\}$. Let $X^{\prime}=\left(X-d_{0}\right) \cup\left\{f_{1}, f_{2}\right\}$ and observe that $r\left(X^{\prime}\right)=5$ and $r_{M \backslash d_{0}}^{*}\left(X^{\prime}\right)=$ $r^{*}\left(X^{\prime} \cup d_{0}\right)-1=4$. Thus $\lambda_{M \backslash d_{0}}\left(X^{\prime}\right)=2$. Also, $e \in \mathrm{cl}_{M \backslash d_{0}}^{*}\left(X^{\prime}\right)$. Towards an application of Lemma 4.2, it remains to show that the pair $\left\{d_{0}, e\right\}$ is $N$ deletable, and $\left|X^{\prime}-(C \cup D)\right| \leq 2$ for an $N$-labelling $(C, D)$ with $\left\{d_{0}, e\right\} \subseteq D$. To this end, observe that $\left\{f_{1}, f_{2}\right\}$ is contained in a 4 -element circuit $C_{5}$ that, by orthogonality with $C_{3}^{*}$ and $C_{4}^{*}$, meets $\left\{d_{1}, d_{0}, e\right\}$ and $\left\{d_{2}, d_{0}, e\right\}$. Since $C_{0}^{*}=\left\{f_{1}, f_{2}, d_{1}, d_{2}\right\}$ is independent, $C_{5}$ meets $\left\{d_{0}, e\right\}$. By orthogonality 
with $C_{1}^{*}$ and $C_{2}^{*}$, there are two cases: if $d_{0} \in C_{5}$, then $C_{5}=\left\{f_{1}, f_{2}, d_{0}, x\right\}$; otherwise, $C_{5}=\left\{f_{1}, f_{2}, e, g\right\}$ for some $g \in E(M)-Y$. In the first case, $M \backslash d_{1} \backslash d_{2} \backslash e / c_{1} / c_{2} / f_{1} / f_{2}$ has an $N$-minor and $\left\{d_{0}, x\right\}$ is a parallel pair in this matroid, so $d_{0}$ is $N$-deletable. In the second case, $M \backslash d_{0} \backslash d_{1} \backslash d_{2} / c_{1} / c_{2} / f_{1} / f_{2}$ has an $N$-minor, and $\{e, g\}$ is a parallel pair in this matroid, so $e$ is $N$ deletable. In either case, (ii) holds by Lemma 4.2 .

Now we may assume, by symmetry, that $s_{1}=c_{2}$ and $s_{2} \in\left\{d_{0}, c_{1}\right\}$. That is, $C_{3}^{*}=\left\{d_{1}, e, c_{2}, f_{1}\right\}$ and either $C_{4}^{*}=\left\{d_{2}, e, d_{0}, f_{2}\right\}$ or $C_{4}^{*}=\left\{d_{2}, e, c_{1}, f_{2}\right\}$. In the latter case, the lemma holds by 4.4.6. So we may assume that $C_{4}^{*}=$ $\left\{d_{2}, e, d_{0}, f_{2}\right\}$.

If $f_{1}=f_{2}$, then, by cocircuit elimination, $\left\{d_{1}, d_{2}, e, c_{2}, d_{0}\right\}$ contains a cocircuit. Since $r^{*}(X)=4$, this cocircuit must contain $e$, in which case $e \in \mathrm{cl}^{*}(X)$; a contradiction. So $f_{1} \neq f_{2}$.

Now $M \backslash d_{0} \backslash d_{1} \backslash d_{2}$ has an $N$-minor, where in this matroid $\left\{c_{1}, c_{2}, x\right\}$ is contained in a series class and $\left\{e, f_{2}\right\}$ is a series pair. Let $C_{1}$ be the 4element circuit containing $\left\{c_{1}, x\right\}$. By orthogonality with $C_{2}^{*}$, this circuit meets $\left\{c_{2}, d_{2}, d_{0}\right\}$. If $c_{2} \in C_{1}$, then $C_{1}$ intersects $C_{4}^{*}$ in at most one element, so $C_{1} \cap C_{4}^{*}=\emptyset$. By orthogonality with $C_{3}^{*}$, the final element is either $d_{1}$ or $f_{1}$. But if $C_{1}=\left\{c_{1}, x, c_{2}, d_{1}\right\}$, then the lemma holds by 4.4.5. So $C_{1}=$ $\left\{c_{1}, x, c_{2}, f_{1}\right\}$. Let $X^{\prime}=C_{0} \cup C_{1}=\left(X-\left\{d_{1}, d_{2}\right\}\right) \cup\left\{e, f_{1}\right\}$. Now $r\left(X^{\prime}\right)=4$ and $r_{M \backslash d_{1}}^{*}\left(X^{\prime}\right) \leq r^{*}(X)=4$, so $\lambda_{M \backslash d_{1}}\left(X^{\prime}\right)=2$. As $d_{2} \in \mathrm{cl}_{M \backslash d_{1}}^{*}\left(X^{\prime}\right)$ and $M \backslash d_{1} \backslash d_{2} \backslash d_{0} / c_{1} / c_{2} / e$ has an $N$-minor, (ii) holds by Lemma 4.2 .

So we may assume that $C_{1}$ meets $\left\{d_{0}, d_{2}\right\}$. Then $\left|C_{1} \cap C_{4}^{*}\right|=2$, by orthogonality. Thus $C_{1}$ intersects $C_{3}^{*}$ in at most one element, so $C_{1} \cap C_{3}^{*}=$ $\emptyset$; in particular, $e \notin C_{1}$. So $C_{1}$ is one of $\left\{c_{1}, x, d_{0}, d_{2}\right\},\left\{c_{1}, x, d_{0}, f_{2}\right\}$ or $\left\{c_{1}, x, d_{2}, f_{2}\right\}$.

If $C_{1}=\left\{c_{1}, x, d_{0}, d_{2}\right\}$, then as $C_{1}$ is coindependent, $\left\{d_{1}, c_{2}\right\} \subseteq \mathrm{cl}^{*}\left(C_{1}\right)$. Recall that $M \backslash d_{1} \backslash d_{2} / c_{1} / c_{2}$ has an $N$-minor, where $\left\{d_{0}, e\right\}$ is a parallel pair in this matroid, so $M \backslash d_{1} \backslash d_{2} \backslash e / c_{1}$ has an $N$-minor. In turn, $\left\{d_{0}, f_{2}\right\}$ is a series pair, so $M \backslash d_{1} / c_{1} / d_{0}$ has an $N$-minor; then $\left\{c_{2}, e\right\}$ is a parallel pair, so $M \backslash d_{1} \backslash c_{2}$ has an $N$-minor. Thus (ii) holds, by Lemma 4.2, when $C_{1}=\left\{c_{1}, x, d_{0}, d_{2}\right\}$. So $C_{1}=\left\{c_{1}, x, d^{\prime}, f_{2}\right\}$ for some $d^{\prime} \in\left\{d_{0}, d_{2}\right\}$. In particular, $f_{2} \in \operatorname{cl}\left(X-\left\{c_{2}, d_{1}\right\}\right)$.

Let $Y=X \cup\left\{e, f_{1}, f_{2}\right\}$. Our goal is to bound $r(Y)$. Note that if $r(Y) \leq 5$, then, as $r^{*}(Y) \leq 5$, it follows that $\lambda(Y) \leq 1$, so $|E(M)| \leq 10$; a contradiction.

Recall that $\{x, e\}$ is $N$-contractible, and consider the circuit $C_{3}$ containing this pair. By orthogonality with $C_{1}^{*}$ and $C_{2}^{*}$, either $d_{0} \in C_{3}$, or $C_{3}$ has an element in $\left\{c_{1}, d_{1}\right\}$ and an element in $\left\{c_{2}, d_{2}\right\}$. In the latter case, by orthogonality with $C_{4}^{*}$ we see that $d_{2} \in C_{3}$. Then, by orthogonality with $C_{3}^{*}$, we see that $d_{1} \in C_{3}$. On the other hand, if $d_{0} \in C_{3}$, then the final element is in $\left\{d_{1}, c_{2}, f_{1}\right\}$, due to the cocircuit $C_{3}^{*}$. But if $C_{3}=\left\{x, e, d_{0}, d_{1}\right\}$, then by circuit elimination with $C_{0}$, there is a circuit contained in $\left\{x, d_{0}, d_{1}, c_{1}, c_{2}\right\}$, which by orthogonality with $C_{4}^{*}$ is $\left\{x, d_{1}, c_{1}, c_{2}\right\}$, so the lemma holds by 
4.4.5. If $C_{3}=\left\{x, e, d_{0}, c_{2}\right\}$, then $C_{0} \cup C_{3}$ is a 5-element plane that intersects $C_{3}^{*}$ in two elements; a contradiction. Therefore we may assume that $C_{3}$ is $\left\{x, e, d_{1}, d_{2}\right\}$ or $\left\{x, e, d_{0}, f_{1}\right\}$.

Suppose that $C_{3}=\left\{x, e, d_{1}, d_{2}\right\}$. Then $d_{1} \in \operatorname{cl}\left(X-d_{1}\right)$. Recall that $M \backslash d_{1} \backslash d_{2} \backslash e$ has an $N$-minor, and observe that $\left\{d_{0}, f_{2}\right\}$ and $\left\{f_{1}, c_{2}\right\}$ are series pairs in this matroid. Thus $\left\{d_{0}, f_{1}\right\}$ is $N$-contractible. The 4 -element circuit $C_{4}$ containing $\left\{d_{0}, f_{1}\right\}$ meets $\left\{e, d_{1}, c_{2}\right\},\left\{e, d_{2}, f_{2}\right\},\left\{x, c_{1}, d_{1}\right\}$ and $\left\{x, c_{2}, d_{2}\right\}$, by orthogonality with $C_{3}^{*}, C_{4}^{*}, C_{1}^{*}$, and $C_{2}^{*}$. Hence $C_{4}$ is either $\left\{d_{0}, f_{1}, e, x\right\}$ or $\left\{d_{0}, f_{1}, d_{1}, d_{2}\right\}$. In either case, $f_{1} \in \operatorname{cl}(X \cup e)$. Recall that $\left\{d_{1}, e, f_{2}\right\} \subseteq \operatorname{cl}\left(X-d_{1}\right)$, so $r(Y) \leq 5$. Now $\lambda(Y) \leq 1$, so $|E(M)| \leq 10$; a contradiction.

So we may assume that $C_{3}=\left\{x, e, d_{0}, f_{1}\right\}$; in particular, $f_{1} \in \operatorname{cl}(X-$ $\left.\left\{d_{1}, d_{2}\right\}\right)$. Now $f_{2}$ and $c_{2}$ are in distinct series pairs of $M \backslash d_{0} \backslash d_{1} \backslash d_{2}$, so $\left\{f_{2}, c_{2}\right\}$ is $N$-contractible. Consider the circuit $C_{5}$ containing $\left\{f_{2}, c_{2}\right\}$. By orthogonality with $C_{3}^{*}, C_{2}^{*}$ and $C_{4}^{*}$, this circuit meets $\left\{d_{1}, e, f_{1}\right\},\left\{x, d_{0}, d_{2}\right\}$ and $\left\{e, d_{0}, d_{2}\right\}$. But if $C_{5}$ meets $C_{1}^{*}$, it does so in two elements; so $C_{5}$ is either $\left\{f_{2}, c_{2}, d_{1}, d_{0}\right\},\left\{f_{2}, c_{2}, e, d_{2}\right\}$ or $\left\{f_{2}, c_{2}, f_{1}, d_{2}\right\}$. In the first case, $c_{2} \in \operatorname{cl}\left(\left(X \cup f_{2}\right)-c_{2}\right)=\operatorname{cl}\left(X-c_{2}\right)$, so $X-c_{2}$ spans $Y$, implying $\lambda(Y) \leq 1$, so $|E(M)| \leq 10$; a contradiction. In the second case, $f_{2} \in \operatorname{cl}\left(\left(X-\left\{x, d_{1}\right\}\right) \cup e\right)=$ $\operatorname{cl}\left(X-\left\{x, d_{1}\right\}\right)$. Due to the circuit $C_{1}$, it follows that $x \in \operatorname{cl}\left(X-\left\{x, d_{1}\right\}\right)$, so $X-\left\{x, d_{1}\right\}$ spans $Y-d_{1}$, implying $r(Y) \leq 5$, so $\lambda(Y) \leq 1$, and $|E(M)| \leq 10$; a contradiction. So we may assume that $C_{5}=\left\{f_{2}, c_{2}, d_{2}, f_{1}\right\}$.

Recall that $C_{1}=\left\{c_{1}, x, d^{\prime}, f_{2}\right\}$ for some $d^{\prime} \in\left\{d_{0}, d_{2}\right\}$. Now, if $d^{\prime}=d_{0}$, then $X-\left\{d_{1}, d_{2}\right\}=\left\{c_{1}, d_{0}, x, c_{2}\right\}$ spans $Y-d_{1}$, in which case $r(Y) \leq 5$, so $\lambda(Y) \leq 1$, and $|E(M)| \leq 10$; a contradiction. So we may also assume that $C_{1}=\left\{c_{1}, x, d_{2}, f_{2}\right\}$.

Let $Y^{\prime}=Y-\left\{d_{1}, d_{2}\right\}$. Now $\left\{c_{1}, c_{2}, d_{0}, x, f_{2}\right\}$ spans $Y^{\prime}$, so $r\left(Y^{\prime}\right) \leq 5$; and $r_{M \backslash d_{2}}^{*}\left(Y^{\prime}\right)=r^{*}\left(Y^{\prime} \cup d_{2}\right)-1=4$, so $\lambda_{M \backslash d_{2}}\left(Y^{\prime}\right)=2$. Moreover, $d_{1} \in$ $\mathrm{cl}_{M \backslash d_{2}}^{*}\left(Y^{\prime}\right)$. We work towards an application of Lemma 4.2, By circuit elimination on $C_{1}$ and $C_{5}$, there is a circuit contained in $\left\{c_{1}, x, c_{2}, f_{1}, d_{2}\right\}$. But this set intersects $C_{4}^{*}$ in a single element, $d_{2}$, so $C_{6}=\left\{c_{1}, x, c_{2}, f_{1}\right\}$ is a circuit. Recall that $M \backslash d_{1} \backslash d_{2} \backslash e / c_{1}$ has an $N$-minor, where $\left\{c_{2}, f_{1}\right\}$ and $\left\{d_{0}, f_{2}\right\}$ are series pairs in this matroid, so $M \backslash d_{1} \backslash d_{2} \backslash e / c_{1} / c_{2} / f_{2}$ has an $N$ minor. Due to the circuit $C_{6}$, the pair $\left\{x, f_{1}\right\}$ is a parallel pair in this matroid. It follows that (ii) holds by Lemma 4.2 .

4.4.8. Suppose $\left|C_{1}^{*} \cap C_{2}^{*}\right|=2$. Then the lemma holds.

Subproof. If $d_{1} \notin C_{2}^{*}$, then the lemma holds by 4.4.7. So we may assume that $d_{1} \in C_{2}^{*}$. Let $C_{1}^{*}=\left\{d_{0}, d_{1}, c_{1}, x\right\}$ and $C_{2}^{*}=\left\{d_{0}, d_{1}, d_{2}, c_{2}\right\}$, and recall that $X=C_{1}^{*} \cup C_{2}^{*}$. As $M \backslash d_{0} \backslash d_{1} \backslash d_{2}$ has an $N$-minor, it follows that there is a 4-element circuit $C_{0}$ containing $\left\{c_{1}, c_{2}\right\}$, and there is a 4-element circuit $C_{1}$ containing $\left\{x, c_{2}\right\}$. If $\left\{c_{1}, c_{2}, x, d_{2}\right\}$ is a circuit, then the lemma holds by 4.4.4. If $\left\{c_{1}, c_{2}, x, d_{1}\right\}$ is a circuit, then we can swap the $N$-labels on $x$ and $d_{1}$, and with $x \in D \cap\left(C_{1}^{*}-C_{2}^{*}\right)$ playing the role of $d_{1}$, the lemma holds by 4.4.7. By symmetry, the lemma also holds if $\left\{c_{1}, c_{2}, x, d_{0}\right\}$ is a circuit. 
Now, if $C_{0}$ and $C_{1}$ are both contained in $X$, then $C_{0} \neq C_{1}$, so $r(X) \leq 4$, implying $\lambda(X)=2$, and (i) holds by 4.4.3. Without loss of generality, $C_{0}$ is not contained in $X$. By orthogonality and symmetry, we may assume that $C_{0}=\left\{c_{1}, c_{2}, d_{1}, e\right\}$ for some $e \in E(M)-X$.

Now $M \backslash d_{0} \backslash d_{2} / c_{1} / c_{2}$ has an $N$-minor, and $\left\{d_{1}, e\right\}$ is a parallel pair in this matroid, so by swapping $N$-labels we may assume that $\left\{d_{0}, e, d_{2}\right\} \subseteq D$. In particular, there exists a 4-element cocircuit $C_{3}^{*}$ containing $\left\{d_{2}, e\right\}$, and a 4element cocircuit $C_{4}^{*}$ containing $\left\{d_{0}, e\right\}$. By orthogonality, each of $C_{3}^{*}$ and $C_{4}^{*}$ meets $\left\{c_{1}, d_{1}, c_{2}\right\}$. We claim that $c_{1} \in C_{3}^{*} \cap C_{4}^{*}$. First suppose $d_{1} \in C_{3}^{*}$. We may assume that $C_{3}^{*} \nsubseteq C_{2}^{*} \cup e$, for otherwise $C_{2}^{*} \cup C_{3}^{*}=C_{2}^{*} \cup e$ is a corank-3 set, contradicting 4.4.2. So $C_{3}^{*}=\left\{d_{2}, e, d_{1}, f\right\}$ for some $f \in E(M)-\left(C_{2}^{*} \cup e\right)$. Now $\left|C_{2}^{*} \cap C_{3}^{*}\right|=2$, with $d_{2} \in C_{2}^{*} \cap C_{3}^{*}, d_{0} \in C_{2}^{*}-C_{3}^{*}$, and $e \in C_{3}^{*}-C_{2}^{*}$, so we can apply 4.4.7, with $C_{3}^{*}$ in the role of $C_{1}^{*}$, in which case the lemma holds. Similarly, if $c_{2} \in C_{3}^{*}$, then $C_{3}^{*} \nsubseteq C_{2}^{*} \cup e$, by 4.4.2, so $C_{3}^{*}=\left\{d_{2}, e, c_{2}, f\right\}$ for some $f \in E(M)-\left(C_{2}^{*} \cup e\right)$. Then, with $C_{3}^{*}$ in the role of $C_{1}^{*}$, the lemma holds by 4.4.7. So $c_{1} \in C_{3}^{*}$. By a similar argument, $c_{1} \in C_{4}^{*}$.

Now let $C_{3}^{*}=\left\{d_{2}, e, c_{1}, f\right\}$ and $C_{4}^{*}=\left\{d_{0}, e, c_{1}, g\right\}$, for some $f \in E(M)-$ $\left\{d_{2}, e, c_{1}\right\}$ and $g \in E(M)-\left\{d_{0}, e, c_{1}\right\}$. Note that $\left|C_{3}^{*} \cap C_{4}^{*}\right| \neq 3$, by 4.4.2. So $C_{3}^{*} \cap C_{4}^{*}=\left\{e, c_{1}\right\}$, with $d_{2} \in C_{3}^{*}-C_{4}^{*}$ and $d_{0} \in C_{4}^{*}-C_{3}^{*}$. Using 4.4.7, with $C_{3}^{*}$ and $C_{4}^{*}$ in the roles of $C_{1}^{*}$ and $C_{2}^{*}$, the lemma holds.

Now, by 4.4.2 and 4.4.8, we may assume that for any triple of elements $d, d^{\prime}, d^{\prime \prime} \in D$, the 4-element cocircuits containing $\left\{d, d^{\prime}\right\},\left\{d, d^{\prime \prime}\right\}$ and $\left\{d^{\prime}, d^{\prime \prime}\right\}$ pairwise intersect in a single element. Dually, for a triple of elements in $C$, two 4-element circuits each containing a pair of this triple meet in a single element.

Let $C_{0}^{*}, C_{1}^{*}$ and $C_{2}^{*}$ be the 4 -element cocircuits containing $\left\{d_{1}, d_{2}\right\}$, $\left\{d_{0}, d_{2}\right\}$, and $\left\{d_{0}, d_{1}\right\}$ respectively, where $\left\{d_{0}, d_{1}, d_{2}\right\} \subseteq D$. Then $\left|C_{i}^{*} \cap C_{j}^{*}\right|=$ 1 for distinct $i, j \in\{0,1,2\}$. Let $\left\{c_{i}, e_{i}\right\} \subseteq C_{i}^{*}$ for each $i \in\{0,1,2\}$, where $c_{0}, c_{1}, c_{2}, e_{0}, e_{1}, e_{2} \in E(M)-\left\{d_{0}, d_{1}, d_{2}\right\}$ are distinct. Since $M \backslash d_{0} \backslash d_{1} \backslash d_{2}$ has an $N$-minor, $M / c_{0} / c_{1} / c_{2}$ has an $N$-minor, up to switching the $N$-labels on $c_{i}$ and $e_{i}$ for each $i$. Thus, for distinct $i, j \in\{0,1,2\}$, the pair $\left\{c_{i}, c_{j}\right\}$ is contained in a 4-element circuit, and these circuits meet in a single element. Let $X=C_{0}^{*} \cup C_{1}^{*} \cup C_{2}^{*}$.

Suppose there is such a circuit that is not contained in $X$. Without loss of generality, let $\left\{c_{1}, c_{2}, g\right\}$ be contained in a 4-element circuit $C_{0}$, for $g \in E(M)-X$. By orthogonality, $C_{0}=\left\{c_{1}, c_{2}, d_{0}, g\right\}$. It now follows that $\left\{g, d_{1}\right\}$ and $\left\{g, d_{2}\right\}$ are $N$-deletable, so they are contained in 4-element cocircuits $C_{3}^{*}$ and $C_{4}^{*}$ respectively. Since $\left|C_{3}^{*} \cap C_{2}^{*}\right|=1$, and by orthogonality, $c_{1} \in C_{3}^{*}$. Similarly, $c_{2} \in C_{4}^{*}$. Now $C_{3}^{*}$ and $C_{4}^{*}$ intersect each of $C_{0}^{*}, C_{1}^{*}$, and $C_{2}^{*}$ in a single element, so $C_{3}^{*}=\left\{g, d_{1}, c_{1}, h_{1}\right\}$ and $C_{4}^{*}=\left\{g, d_{2}, c_{2}, h_{2}\right\}$ for some distinct $h_{1}, h_{2} \in E(M)-(X \cup g)$.

Now consider the 4-element circuit $C_{1}$ containing $\left\{c_{0}, c_{1}\right\}$. By orthogonality with $C_{1}^{*}$, this circuit meets $\left\{d_{0}, d_{2}, e_{1}\right\}$. If $d_{0} \in C_{1}$, then, by orthogonality with $C_{0}^{*}$ and $C_{2}^{*}$, we have $d_{1} \in C_{1}$. If $d_{2} \in C_{1}$, then, by orthogonality with 
$C_{3}^{*}$ and $C_{4}^{*}$, we have $g \in C_{1}$. If $e_{1} \in C_{1}$, then, by orthogonality with $C_{0}^{*}$ and $C_{3}^{*}$, we have $d_{1} \in C_{1}$, but then $\left|C_{1} \cap C_{2}^{*}\right|=1$; a contradiction. So $C_{1}=\left\{c_{0}, c_{1}, d_{0}, d_{1}\right\}$ or $C_{1}=\left\{c_{0}, c_{1}, d_{2}, g\right\}$, but in either case, $\left|C_{0} \cap C_{1}\right|=2$; a contradiction.

So, for distinct $i, j \in\{0,1,2\}$, the 4-element circuit $C_{i, j}$ containing $\left\{c_{i}, c_{j}\right\}$ is contained in $X$. Let $\{i, j, k\}=\{0,1,2\}$. Now, $C_{i, j}$ cannot meet $\left\{c_{k}, e_{k}\right\}$, by orthogonality, as there is no element common to $C_{i}^{*}, C_{j}^{*}$ and $C_{k}^{*}$. Then, by orthogonality with $C_{k}^{*}$, either $C_{i, j}$ contains $\left\{d_{i}, d_{j}\right\}$, or it does not meet this pair, for any $\{i, j, k\}=\{0,1,2\}$. But now if $C_{i, j}$ meets $\left\{d_{0}, d_{1}, d_{2}\right\}$, then neither $C_{i, k}$ nor $C_{j, k}$ meets $\left\{d_{0}, d_{1}, d_{2}\right\}$, since the circuits pairwise intersect in a single element, in which case $C_{i, k}$ and $C_{j, k}$ intersect in the pair $\left\{c_{k}, e_{k}\right\}$; a contradiction. So $C_{i, j}=\left\{c_{i}, e_{i}, c_{j}, e_{j}\right\}$ for all distinct $i, j \in\{0,1,2\}$, which again contradicts that these circuits pairwise intersect in one element. This completes the proof.

Proof of Proposition 4.1. If, up to switching $N$-labels, there exists a pair $\left\{c_{1}, c_{2}\right\} \subseteq C$ or $\left\{d_{1}, d_{2}\right\} \subseteq D$ that is contained in a quad, then the proposition holds by Lemma 4.3. Otherwise, the proposition holds by Lemma 4.4

\section{PARTiCUlar 3-SEPARATORS}

Now we show that when $M$ has a particular 3 -separator $P$, most of the elements that are $N$-labelled for removal must be in $P$, otherwise $M$ has an $N$-detachable pair.

As a warm-up, we first consider the case where $M$ has an augmented quad 3-separator $Q \cup z$. We will later prove a similar result, Lemma 5.4, that handles the case where $M$ has one of the other particular 3-separators.

Lemma 5.1. Let $M$ be a 3-connected matroid, and let $N$ be a 3-connected minor of $M$ such that $|E(N)| \geq 4$, and every triangle or triad of $M$ is $N$-grounded. Suppose that $(Q,\{z\}, S)$ is a cyclic 3-separation of $M$ such that $Q \cup z$ is an augmented quad 3-separator, $M \backslash z$ has an $N$-minor with $|Q \cap E(N)| \leq 1$, and $M \backslash s$ is not 3-connected for each $s \in S$ that is $N$ deletable in $M \backslash z$. If $M$ has no $N$-detachable pairs, then $S \subseteq E(N)$.

Proof. Since $M \backslash z$ has an $N$-minor with $|Q \cap E(N)| \leq 1$, it follows from Lemma 2.11 that, for any distinct $q, q^{\prime} \in Q$, the matroid $M \backslash z \backslash q / q^{\prime}$ has an $N$-minor. In particular, each element of $Q$ is $N$-flexible in $M \backslash z$. Since every triangle or triad of $M$ is $N$-grounded, observe that no triangle or triad meets $Q$. Let $(C, D)$ be an $N$-labelling such that $z \in D$ and at most one element of $Q$ is not $N$-labelled for removal. Let $Q=\left\{q_{1}, q_{2}, q_{3}, q_{4}\right\}$.

First we claim that if $e \in S$ is $N$-labelled for contraction, then $M / e$ is not 3 -connected. Towards a contradiction, suppose that $M / e$ is 3 -connected for some $e \in S$ that is $N$-labelled for contraction. If $e \in \operatorname{cl}(Q)$, then $\operatorname{si}(M / e)$ is not 3-connected, so we may assume that $e \notin \operatorname{cl}(Q)$. As $Q$ is a quad in $M / e$, the matroid $\operatorname{si}\left(M / e / q_{i}\right)$ is 3-connected for each $i \in[4]$, by Lemma 2.8, It 
follows from Lemma 2.11 that any such pair $\left\{e, q_{i}\right\}$ is $N$-contractible in $M \backslash z$. Thus, as $M$ has no $N$-detachable pairs, for each $i \in[4]$ there is a 4-element circuit containing $\left\{e, q_{i}\right\}$. By orthogonality, and since $e \notin \operatorname{cl}(Q)$, each of these circuits intersects $Q$ in precisely two elements. We may assume that $\left\{q_{1}, q_{2}, e, h\right\}$ is a circuit, for some $h \in E(M)-(Q \cup e)$. Now, for $i \in\{1,2\}$ and $j \in\{3,4\}$, the matroid $M \backslash z \backslash q_{j} / q_{i} / e$ has an $N$-minor. Note that if $h=z$, then $(Q \cup z, e, S-e)$ is a vertical 3-separation, so $\operatorname{si}(M / e)$ is not 3connected; a contradiction. Thus $h$ is $N$-deletable in $M \backslash z$. By hypothesis, $M \backslash h$ is not 3-connected. Since each triad of $M$ is $N$-grounded, $h$ is not in a triad, so $\operatorname{co}(M \backslash h)$ is not 3-connected, and $M$ has a cyclic 3-separation $(U,\{h\}, V)$ with $|U \cap Q| \geq 2$. Then, by uncrossing, $(U \cup Q,(V-Q) \cup h)$ is 3-separating. If $e \in U$, then $h \in \operatorname{cl}(U \cup Q) \cap \operatorname{cl}^{*}(U)$, so $\lambda(U \cup Q \cup h) \leq 1$. Hence $|V-Q| \leq 1$, so $|V|=3$. But then $V$ is a triangle containing an $N$ contractible element; a contradiction. So $e \in V$. Since $e$ is $N$-contractible, it follows that $|V| \geq 4$. Now $U \cup Q$ and $U \cup(Q \cup e)$ are exactly 3-separating, so $(U \cup Q,\{e\},(V \cup h)-(Q \cup e))$ is a path of 3-separations where $e$ is a guts element. As $e$ is not in a triangle, $r((V \cup h)-(Q \cup e)) \geq 3$, so the path of 3 -separations is a vertical 3-separation, implying $\mathrm{si}(M / e)$ is not 3-connected; a contradiction.

Now, if $e \in S$ is $N$-labelled for removal, then either $M / e$ is not 3-connected and $e$ is $N$-labelled for contraction, or $M \backslash e$ is not 3-connected and $e$ is $N$ labelled for deletion.

Suppose there is some $e \in S-\operatorname{cl}(Q)$ that is $N$-labelled for contraction. By the foregoing, $M / e$ is not 3 -connected. Moreover, $e$ is not in a triangle, so $\operatorname{si}(M / e)$ is not 3 -connected, and hence $M$ has a vertical 3-separation $(U,\{e\}, V)$ with $|U \cap E(N)| \leq 1$. We may assume, without loss of generality, that $V \cup e$ is closed. By the dual of Lemma 2.12, at most one element in $U$ is not $N$-flexible in $M / e$, and if such an element $x$ exists, then $x \in U \cap \mathrm{cl}^{*}(V)$ and $e \in \operatorname{cl}(U-x)$. But if $u \in S \cup z$ is $N$-flexible, then, as either $\operatorname{si}(M / u)$ or $\operatorname{co}(M \backslash u)$ is 3-connected by Bixby's Lemma, and $u$ is not in an $N$-grounded triangle or triad, either $M / u$ or $M \backslash u$ is 3-connected; a contradiction. So every $N$-flexible element is contained in $Q$, implying $U \subseteq Q$ or $U-x \subseteq Q$ for some $x \in U$ such that $e \in \operatorname{cl}(U-x)$. In either case, $e \in \operatorname{cl}(Q)$; a contradiction.

By a similar argument, if $e \in S$ is $N$-labelled for deletion, then $e \in \operatorname{cl}^{*}(Q)$. Suppose there is some $e \in S \cap \operatorname{cl}^{*}(Q)$. Then $(Q \cup e, z, S-e)$ is a cyclic 3separation, and $e$ is $N$-contractible, by Lemma 2.12(ii), since $e \in \operatorname{cl}^{*}(Q)$. $\operatorname{But}(Q, e,(S-e) \cup z)$ is also a cyclic 3 -separation, and it follows that $M / e$ is 3 -connected; a contradiction. Similarly, if there is an element $s \in S \cap \operatorname{cl}(Q)$, then $(Q \cup s, z, S-s)$ is a cyclic 3-separation, so $M \backslash z$ is 3-connected, and $s$ is $N$-deletable in $M \backslash z$ by Lemma 2.12, a contradiction. We deduce that no elements of $S$ are $N$-labelled for removal, thus completing the proof. 
We now shift our attention to particular 3-separators other than the quad. The next lemma describes how triangles (or, dually, triads) can meet such a particular 3-separator.

Lemma 5.2. Let $M$ be a 3-connected matroid with $P \subseteq E(M)$. Suppose that $P$ contains no triangles, and $P$ has a partition $\left(L_{1}, L_{2}, \ldots, L_{t}\right)$ into pairs, for some $t \geq 3$, such that $L_{i} \cup L_{j}$ is a cocircuit for all distinct $i, j \in[t]$ except perhaps $\{i, j\}=\{1,2\}$. If a triangle $T$ meets $L_{i}$, for some $i \in[t]$, then $L_{i} \subseteq T$.

Proof. Suppose $T$ is a triangle such that $\left|T \cap L_{i}\right|=1$ for some $i \in[t]$. If $i \notin\{1,2\}$, then, by orthogonality, $T$ meets $L_{j}$ for each $j \in[t]-i$. On the other hand, if $i \in\{1,2\}$, then $T$ meets $L_{t}$. If $T \nsubseteq L_{i} \cup L_{t}$, then $\left|T \cap L_{t}\right|=1$ and, in turn, $T$ meets $L_{j}$ for each $j \in[t-1]$. Since $t \geq 3$, we deduce in either case that the triangle $T$ is contained in $P$; a contradiction.

The next lemma guarantees that when a single element in a particular 3-separator is removed, 3-connectivity is preserved up to series or parallel classes. We require the following in the proof of this lemma.

A set $X$ in a matroid $M$ is fully closed if it is closed and coclosed; that is, $\operatorname{cl}(X)=X=\mathrm{cl}^{*}(X)$. The full closure of a set $X$, denoted $\mathrm{fcl}(X)$, is the intersection of all fully closed sets that contain $X$. We say that a 2 separation $(U, V)$ is trivial if $U$ or $V$ is contained in a series or parallel class. It is easily seen that if $(U, V)$ is a non-trivial 2-separation of a connected matroid $M$, then $(\mathrm{fcl}(U), V-\mathrm{fcl}(U))$ is also a 2-separation of $M$.

Lemma 5.3. Let $M$ be a 3-connected matroid, and let $P$ be either

(a) a spike-like 3-separator or a double-quad 3-separator; or

(b) an elongated-quad 3-separator, a skew-whiff 3-separator, or a twisted-cube-like 3-separator of $M$ or $M^{*}$, with $|E(M)-P| \geq 3$.

Then $\operatorname{si}(M / p)$ and $\operatorname{co}(M \backslash p)$ are 3 -connected for each $p \in P$.

Proof. In case (a), every element of $P$ is in a quad, and the result follows from Lemma 2.8 and its dual. For (b), we consider each of the particular 3 -separators in turn.

5.3.1. The lemma holds when $P$ is a skew-whiff 3-separator or an elongatedquad 3-separator.

Subproof. Suppose that $P$ is a skew-whiff 3-separator. By symmetry and duality, it suffices to show that $\operatorname{co}(M \backslash p)$ is 3-connected for some $p \in P$. Let $P=\left\{u_{1}, u_{2}, c, d, v_{1}, v_{2}\right\}$ where $\left\{u_{1}, u_{2}, d, v_{1}\right\},\left\{u_{1}, c, d, v_{2}\right\}$, and $\left\{u_{2}, c, v_{1}, v_{2}\right\}$ are circuits, and $\left\{u_{1}, u_{2}, c, d\right\},\left\{u_{1}, u_{2}, v_{1}, v_{2}\right\}$, and $\left\{c, d, v_{1}, v_{2}\right\}$ are cocircuits.

Towards a contradiction, suppose that $M \backslash d$ has a non-trivial 2-separation $(U, V)$. Without loss of generality, $\left|\left\{u_{1}, u_{2}, c\right\} \cap U\right| \geq 2$, and $U$ is fully closed. So $\left\{u_{1}, u_{2}, c\right\} \subseteq U$. If $U$ meets $\left\{v_{1}, v_{2}\right\}$, then $d \in \operatorname{cl}(U)$, so $(U \cup d, V)$ is a 2-separation of $M$; a contradiction. So $\left\{v_{1}, v_{2}\right\} \subseteq V$. Now $c \in \mathrm{cl}_{M \backslash d}^{*}(V)$, so $(U-c, V \cup c)$ is also a 2-separation of $M \backslash d$. Recall that $|E(M)-P| \geq 3$. 
If $|V-P| \leq 1$, then $|U-P| \geq 2$, so we may assume, up to relabelling, that $|V-P| \geq 2$.

Let $C_{1}$ be the circuit $\left\{u_{2}, c, v_{1}, v_{2}\right\}$. Observe that $\lambda_{M \backslash d}\left(C_{1}\right)=2$, since $d \in \mathrm{cl}^{*}\left(C_{1}\right)$. By submodularity of the connectivity function,

$$
\begin{aligned}
\lambda_{M \backslash d}\left(U \cup C_{1}\right) & \leq \lambda_{M \backslash d}(U)+\lambda_{M \backslash d}\left(C_{1}\right)-\lambda_{M \backslash d}\left(U \cap C_{1}\right) \\
& =1+2-2=1,
\end{aligned}
$$

so $\left(U \cup\left\{v_{1}, v_{2}\right\}, V-\left\{v_{1}, v_{2}\right\}\right)$ is a 2-separation in $M \backslash d$. But $c \in \mathrm{cl}_{M \backslash d}^{*}(U \cup$ $\left.\left\{v_{1}, v_{2}\right\}\right)$, so $\left(U \cup\left\{v_{1}, v_{2}, c\right\}, V-\left\{v_{1}, v_{2}\right\}\right)$ is also a 2-separation of $M$; a contradiction. So $M \backslash d$ has no non-trivial 2-separations, implying $\operatorname{co}(M \backslash d)$ is 3-connected, as required.

A similar argument applies in the case that $P$ is an elongated-quad 3separator; we omit the details.

5.3.2. The lemma holds when $P$ is a twisted-cube-like 3-separator of $M$ or $M^{*}$.

Subproof. It suffices to show that both $\operatorname{si}(M / p)$ and $\operatorname{co}(M \backslash p)$ are 3-connected for each $p \in P$ when $P$ is a twisted-cube-like 3-separator of $M$. Let $P=$ $\left\{s_{1}, s_{2}, t_{1}, t_{2}, q_{1}, q_{2}\right\}$ where $\left\{s_{1}, s_{2}, q_{1}, q_{2}\right\},\left\{t_{1}, t_{2}, q_{1}, q_{2}\right\}$, and $\left\{s_{1}, s_{2}, t_{1}, t_{2}\right\}$ are circuits, and $\left\{s_{1}, t_{1}, q_{1}, q_{2}\right\}$ and $\left\{s_{2}, t_{2}, q_{1}, q_{2}\right\}$ are the non-spanning cocircuits contained in $P$. A similar approach works here as in 5.3.1 we outline the proof, omitting some of the details.

Suppose $M \backslash p$ has a non-trivial 2-separation $(U, V)$, for some $p \in\left\{q_{1}, q_{2}\right\}$. By symmetry, we may assume $p=q_{2}$. Then $\left\{s_{1}, t_{1}, u\right\} \subseteq U$ and $\left\{s_{2}, t_{2}\right\} \subseteq V$, and $|V-P| \geq 2$. By an uncrossing argument with the set $\left\{s_{1}, s_{2}, t_{1}, t_{2}\right\}$, which is 3 -separating in $M \backslash q_{2}$, we deduce that $\left(U \cup\left\{s_{2}, t_{2}\right\}, V-\left\{s_{2}, t_{2}\right\}\right)$ is a 2-separation in $M \backslash q_{2}$, with $q_{2} \in \operatorname{cl}_{M}\left(U \cup\left\{s_{2}, t_{2}\right\}\right)$; a contradiction. So $\operatorname{co}(M \backslash p)$ is 3-connected for $p \in\left\{q_{1}, q_{2}\right\}$.

Suppose $M / p$ has a non-trivial 2-separation $(U, V)$, for some $p \in$ $\left\{s_{1}, s_{2}, t_{1}, t_{2}\right\}$. By symmetry, we may assume $p=t_{2}$. Then, we may assume that $\left|\left\{q_{1}, q_{2}, t_{1}\right\} \cap U\right| \geq 2$ and $U$ is fully closed, but $P-t_{2} \subseteq$ $\mathrm{fcl}_{M / t_{2}}\left(\left\{q_{1}, q_{2}, t_{1}\right\}\right)$, so $P-t_{2} \subseteq U$, and thus $t_{2} \in \mathrm{cl}_{M}^{*}(U)$; a contradiction. So $\operatorname{si}(M / p)$ is 3 -connected for $p \in\left\{s_{1}, s_{2}, t_{1}, t_{2}\right\}$.

Suppose $M / p$ has a non-trivial 2-separation $(U, V)$, for some $p \in\left\{q_{1}, q_{2}\right\}$. By symmetry, we may assume $p=q_{2}$. Then, we may assume that $\left\{s_{1}, s_{2}, q_{1}\right\} \subseteq U$ and $\left\{t_{1}, t_{2}\right\} \subseteq V$, and $|V-P| \geq 2$. By an uncrossing argument with $P-q_{2}$, which is 3 -separating in $M / q_{2}$, we deduce that $\left(U \cup\left\{t_{1}, t_{2}\right\}, V-\left\{t_{1}, t_{2}\right\}\right)$ is a 2-separation in $M / q_{2}$, with $q_{2} \in \mathrm{cl}^{*}\left(U \cup\left\{t_{1}, t_{2}\right\}\right)$; a contradiction. So $\operatorname{si}(M / p)$ is 3-connected for $p \in\left\{q_{1}, q_{2}\right\}$.

Suppose $M \backslash p$ has a non-trivial 2-separation $(U, V)$, for some $p \in$ $\left\{s_{1}, s_{2}, t_{1}, t_{2}\right\}$. By symmetry, we may assume $p=t_{2}$. Then, we may assume that $\left\{s_{2}, q_{1}, q_{2}\right\} \subseteq U$ and $\left\{s_{1}, t_{1}\right\} \subseteq V$. The set $P-t_{2}$ is 3 -separating in $M / t_{2}$. By an uncrossing argument with $U$, when $|V-P| \geq 2$, or with $V$, when $|U-P| \geq 2$, we obtain a 2-separation of $M / t_{2}$ where $t_{2}$ is in the closure of one side; a contradiction. So $\operatorname{si}(M \backslash p)$ is 3 -connected for $p \in\left\{s_{1}, s_{2}, t_{1}, t_{2}\right\}$. $\triangleleft$ 
Recall that when $P$ is a particular 3 -separator with $|E(M)-P| \geq 3$, and $z \in \operatorname{cl}(P)-P$ or $z \in \mathrm{cl}^{*}(P)-P$, we say $P \cup z$ is an augmentation of $P$. The next lemma shows that when a matroid $M$ with no $N$-detachable pairs has a particular 3-separator $P$, either $E(M)-E(N) \subseteq P$, or there is an augmentation $P \cup z$ of $P$ such that $E(M)-E(N) \subseteq P \cup z$. The proof follows a similar approach to Lemma 5.1, but there are some extra subtleties to handle.

Let $N$ be a 3 -connected minor of $M$. An augmented 3 -separator $P \cup z$ is problematic with respect to $N$ if

(a) $z \in \operatorname{cl}(P)$ and $z$ is $N$-contractible but not $N$-deletable, or $z \in \mathrm{cl}^{*}(P)$ and $z$ is $N$-deletable but not $N$-contractible; and

(b) $E(M)-E(N) \subseteq P \cup z$.

Lemma 5.4. Let $M$ be a 3-connected matroid, and let $N$ be a 3-connected minor of $M$ such that $|E(N)| \geq 4$, and every triangle or triad of $M$ is $N$ grounded. Suppose that there exists $d \in E(M)$ such that $M \backslash d$ is 3-connected and has a cyclic 3-separation $\left(Y,\left\{d^{\prime}\right\}, Z\right)$ where $M \backslash d \backslash d^{\prime}$ has an $N$-minor with $|Y \cap E(N)| \leq 1$, and there is a subset $X$ of $Y$ such that for some $c \in \mathrm{cl}_{M \backslash d}^{*}(X)-X$, one of the following holds:

(a) $X \cup\{c, d\}$ is contained in a spike-like 3-separator $P$ of $M$, where $P$ is maximal subject to $|P \cap E(N)| \leq 1$,

(b) $P=X \cup\{c, d\}$ is a skew-whiff 3-separator of $M$,

(c) $P=X \cup\{c, d\}$ is a twisted-cube-like 3-separator of $M$ or $M^{*}$,

(d) $P=X \cup\{c, d\}$ is an elongated-quad 3-separator of $M$, or

(e) $P=X \cup\{a, b, c, d\}$ is a double-quad 3-separator of $M$ with associated partition $\{X,\{a, b, c, d\}\}$ for some distinct $a, b \in E(M)-(X \cup\{c, d\})$.

If $M$ has no $N$-detachable pairs, then either

(i) $|E(M)| \leq 10$ or $M$ is a quad-flower,

(ii) $E(M)-E(N) \subseteq P$, or

(iii) there is some $z \in E(M)-P$ such that $P \cup z$ is an augmentation of $P$ that is problematic with respect to $N$.

Proof. The set $P$ is a particular 3-separator of $M$ that is either a spikelike 3-separator, a skew-whiff 3-separator, an elongated-quad 3-separator, a double-quad 3-separator, or a twisted-cube-like 3-separator of $M$ or $M^{*}$. By definition, each of these particular 3-separators has a partition $\left(L_{1}, L_{2}, \ldots, L_{t}\right)$, for some $t \geq 3$, such that $\left|L_{i}\right|=2$ for each $i \in[t]$, and $L_{i} \cup L_{j}$ is a cocircuit for all distinct $i, j \in[t]$, except when $P$ is a twistedcube-like 3 -separator of $M$ in which case $L_{i} \cup L_{j}$ is a cocircuit for all distinct $i, j \in[t]$ except $\{i, j\}=\{1,2\}$. Dually, $P$ has a partition $\left(K_{1}, K_{2}, \ldots, K_{t}\right)$ such that $\left|K_{i}\right|=2$ for each $i \in[t]$, and $K_{i} \cup K_{j}$ is a circuit for all distinct $i, j \in[t]$, except when $P$ is a twisted-cube-like 3-separator of $M^{*}$ in which case $K_{i} \cup K_{j}$ is a circuit for all distinct $i, j \in[t]$ except $\{i, j\}=\{1,2\}$. 
Let $\left(L_{1}, \ldots, L_{t}\right)$ and $\left(K_{1}, \ldots, K_{t}\right)$ be the partitions of $P$ as described. For the arguments that follow, we require a particularly convenient $N$-labelling, as described in 5.4.2. We start with the following:

5.4.1. There is an $N$-labelling such that $|P \cap E(N)| \leq 1$ when $P$ is not a double-quad 3-separator, and $|P \cap E(N)| \leq 2$ otherwise.

Subproof. Suppose $P$ is not a double-quad 3-separator. Clearly $|P \cap E(N)| \leq$ 1 when $c \in Y \cup d^{\prime}$, since $|Y \cap E(N)| \leq 1$ in $M \backslash d \backslash d^{\prime}$. So assume that $c \in Z$. As $(Y, Z)$ is a 2-separation of $M \backslash d \backslash d^{\prime}$, and $c \in \mathrm{cl}_{M \backslash d}^{*}(X)$, it follows that $(Y \cup c, Z-c)$ is also a 2-separation of $M \backslash d \backslash d^{\prime}$. Note that $|(Z-c) \cap E(N)|>1$, since $|Y \cap E(N)| \leq 1$ and $|E(N)| \geq 4$. Hence, by Lemma 2.11, $\mid(Y \cup c) \cap$ $E(N) \mid \leq 1$ in $M \backslash d$, and $|P \cap E(N)| \leq 1$ as required.

Now suppose $P$ is a double-quad 3-separator. If $d^{\prime} \notin\{a, b, c\}$, then $(Y \cup$ $c, Z-c)$ is a 2-separation of $M \backslash d \backslash d^{\prime}$, and, in the same manner, we deduce that $|(Y \cup c) \cap E(N)| \leq 1$ in $M \backslash d \backslash d^{\prime}$. Moreover, up to swapping $a$ and $b$, the partition $(Y,\{c\},\{b\},\{a\}, Z-\{a, b, c\})$ is a path of 2-separations in $M \backslash d \backslash d^{\prime}$ and, iteratively repeating this process, we deduce that $\mid(Y \cup\{a, b, c\}) \cap$ $E(N) \mid \leq 1$ in $M \backslash d$, as required. On the other hand, if $d^{\prime} \in\{a, b, c\}$, then $|X \cap E(N)| \leq 1$ in $M \backslash d \backslash d^{\prime}$, and $\{a, b, c\}-d^{\prime}$ is a series pair in this matroid, so $|P \cap E(N)| \leq 2$, as required.

5.4.2. There is an $N$-labelling $(C, D)$ such that, up to $N$-label switches on elements in $P$,

(i) for every $i \in[t]$ except perhaps $s \in[t]$, there is an element in $L_{i}$ that is $N$-labelled for contraction; and

(ii) for every $i \in[t]$ except perhaps $s^{\prime} \in[t]$, there is an element in $K_{i}$ that is $N$-labelled for deletion.

Moreover, if such an $s$ exists, then $P$ is an elongated-quad 3-separator or a twisted-cube-like 3-separator of $M$ or $M^{*}$, and $P-L_{s}$ is independent; while if such an $s^{\prime}$ exists, then $P$ is an elongated-quad 3-separator or a twistedcube-like 3-separator of $M$ or $M^{*}$, and $P-K_{s^{\prime}}$ is coindependent.

Subproof. Let $(C, D)$ be an $N$-labelling such that at most two elements of $P$ are $N$-labelled for removal. Suppose $(C, D)$ does not satisfy 5.4.2(i). Then there exist distinct $i, s \in[t]$ such that $L_{i} \cup L_{s}$ does not contain an element that is $N$-labelled for contraction. Then $L_{i} \cup L_{s}$ contains at least two elements that are $N$-labelled for deletion. Apart from when $P$ is a twisted-cube-like 3 -separator of $M$ and $\{i, s\}=\{1,2\}$, the set $L_{i} \cup L_{s}$ is a 4 -element cocircuit, so there is, up to an $N$-label switch, an element in $L_{i} \cup L_{s}$ that is $N$-labelled for contraction, as required. In the exceptional case, $|P \cap E(N)| \leq 1$, so $L_{1} \cup L_{2}$ contains at least three elements that are $N$-labelled for deletion, and $L_{1} \cup L_{2}$ is contained in a 5-element cocircuit; so, again, an element in $L_{i} \cup L_{s}$ is $N$-labelled for contraction after an $N$-label switch. This proves 5.4.2(i). We obtain 5.4.2(ii) by a dual argument.

Suppose that $P$ is an elongated-quad 3-separator or twisted-cube-like 3separator of $M$, so $|P \cap E(N)| \leq 1$. For ease of notation, let $s=t=3$; 
that is, for each $i \in\{1,2\}$ there is an element in $L_{i}$ that is $N$-labelled for contraction. Towards a contradiction, suppose that $P-L_{3}$ is a circuit (when $P$ is an elongated-quad 3-separator, $P-L_{3}$ is a quad; whereas when $P$ is a twisted-cube-like 3 -separator, $P-L_{3}$ is a coindependent circuit). As $|P \cap E(N)| \leq 1$, there is an element $d_{3} \in L_{3}$ that is $N$-labelled for deletion. Let $L_{3}=\left\{d_{3}, x\right\}$. There are elements $c_{1} \in L_{1}$ and $c_{2} \in L_{2}$ that are $N$ labelled for contraction. Since $P-L_{3}$ is a circuit, up to an $N$-label switch we can locate an element $d_{1} \in P-L_{3}$ that is $N$-labelled for deletion. Since $M \backslash d_{3} \backslash d_{1}$ has an $N$-minor, and $x$ is in a series pair in this matroid, we can perform an $N$-label switch so that $x \in L_{3}$ is $N$-labelled for contraction, as required. A similar argument applies in the dual.

In the case that $P$ is a double-quad 3 -separator or spike-like 3-separator, then $P-L_{i}$ contains a circuit for any $i \in[t]$, and a similar argument applies. Finally, if $P$ is a skew-whiff 3 -separator, then it is readily checked that starting from any $N$-labelling satisfying [5.4.2, we can, by performing $N$ label switches, obtain an $N$-labelling where, for each $i \in[3]$, the pair $L_{i}$ contains an element that is $N$-labelled for contraction.

The next claim follows from 5.4.1, Lemma 5.2 and its dual, and the fact that every triangle or triad of $M$ is $N$-grounded. We omit the details.

5.4.3. No element of $P$ is in a triangle or triad of $M$.

Let $(C, D)$ be the $N$-labelling described in 5.4.2, Assume that $M$ has no $N$-detachable pairs. If $r(E(M)-P) \leq 2$, then $Z$ contains a triangle, which, by 5.4.3, is disjoint from $P$. So $|E(M)-P| \geq 3$.

5.4.4. Suppose that there exists some $e \in E(M)-\operatorname{cl}(P)$ that is $N$-labelled for contraction. If $M / e$ is 3-connected, then, for each $i \in[t]-s$, there exists an element $g_{i}$ such that $L_{i} \cup\left\{e, g_{i}\right\}$ is a circuit, where $g_{i}$ is $N$-deletable.

Subproof. Since $e \notin \operatorname{cl}(P)$, we have that $P$ is a particular 3 -separator of $M / e$. For each $i \in[t]-s$, as $L_{i}$ contains an element $x$ that is $N$-labelled for contraction, Lemma 5.3 implies that either $M / e / x$ is 3-connected, in which case $\{e, x\}$ is an $N$-detachable pair, or $x$ is in a triangle of $M / e$. As we are under the assumption that $M$ has no $N$-detachable pairs, $L_{i}$ is contained in a triangle of $M / e$ for each $i \in[t]-s$, by Lemma [5.2. As each $L_{i}$ is not contained in a triangle in $M$, by 5.4.3, there exists an element $g_{i}$ such that $L_{i} \cup\left\{e, g_{i}\right\}$ is a circuit of $M$. Now, as $M / e / x$ has an $N$-minor and $g_{i}$ is in a parallel pair in this matroid, $g_{i}$ is $N$-deletable.

We obtain the following by a dual argument:

5.4.5. Suppose that there exists some $e \in E(M)-\mathrm{cl}^{*}(P)$ that is $N$-labelled for deletion. If $M \backslash e$ is 3-connected, then, for each $i \in[t]-s^{\prime}$, there exists an element $h_{i}$ such that $K_{i} \cup\left\{e, h_{i}\right\}$ is a cocircuit, where $h_{i}$ is $N$-labelled for contraction up to an $N$-label switch.

Next we prove the following: 
5.4.6. If there exists an element $e \in E(M)-P$ that is $N$-labelled for contraction, then either $\operatorname{si}(M / e)$ is not 3-connected, $|E(M)| \leq 10$, or $M$ is a quad-flower.

Subproof. Suppose there exists an element $e \in \operatorname{cl}(P)-P$ that is $N$-labelled for contraction. Let $Q=E(M)-(P \cup e)$, and observe that $(P,\{e\}, Q)$ is a path of 3-separations with $|Q| \geq 2$. If $r(Q) \leq 2$, then $e$ is an $N$-contractible element in a triangle; a contradiction. So $r(Q) \geq 3$, in which case the path of 3 -separations is a vertical 3-separation, implying $\mathrm{si}(M / e)$ is not 3-connected, as required.

Suppose $e \in E(M)-\operatorname{cl}(P)$ and $\operatorname{si}(M / e)$ is 3-connected. As $e$ is $N$ contractible, it is not in an $N$-grounded triangle, so $M / e$ is 3 -connected. By 5.4.4, for each $i \in[t]-s$ there exists an element $g_{i}$ such that $L_{i} \cup\left\{e, g_{i}\right\}$ is a circuit, and $g_{i}$ is $N$-labelled for deletion up to an $N$-label switch.

Suppose $\operatorname{co}\left(M \backslash g_{i}\right)$ is not 3-connected for some $i \in[t]-s$. We claim that there is a cyclic 3-separation $\left(U,\left\{g_{i}\right\}, V\right)$ of $M$ such that $L_{i} \subseteq U$. There certainly exists some cyclic 3-separation $\left(U^{\prime},\left\{g_{i}\right\}, V^{\prime}\right)$ of $M$. Suppose $L_{i}$ meets both $U^{\prime}$ and $V^{\prime}$; let $L_{i}=\{u, v\}$ with $u \in U^{\prime}$ and $v \in V^{\prime}$. If $\left|P \cap U^{\prime}\right|=1$, then $u \in \operatorname{cl}\left(V^{\prime}\right)$, so $\left(V^{\prime} \cup u,\left\{g_{i}\right\}, U^{\prime}-u\right)$ is a cyclic 3-separation of $M$ with $L_{i} \subseteq V^{\prime} \cup u$. Similarly, if $\left|P \cap V^{\prime}\right|=1$, then $\left(U^{\prime} \cup v,\left\{g_{i}\right\}, V^{\prime}-v\right)$ is a cyclic 3-separation of $M$ with $L_{i} \subseteq U^{\prime} \cup v$. Now assume that $\left|P \cap U^{\prime}\right| \geq 2$ and $\left|P \cap V^{\prime}\right| \geq 2$. As $|E(M)-P| \geq 3$, we may assume, without loss of generality, that $\left|V^{\prime}-P\right| \geq 2$. By uncrossing, $\left(U^{\prime} \cup P,\left\{g_{i}\right\}, V^{\prime}-P\right)$ is a path of 3-separations. If $r^{*}\left(V^{\prime}-P\right) \leq 2$, then $g_{i}$ is in a triad; a contradiction. We deduce that there exists a cyclic 3 -separation $\left(U,\left\{g_{i}\right\}, V\right)$ with $L_{i} \subseteq U$, as claimed.

Now, if $e \in U$, then $g_{i} \in \operatorname{cl}(U)$, so $(U, V)$ is a 2-separation of $M$; a contradiction. So $e \in V$. As $e \in \operatorname{cl}\left(U \cup g_{i}\right)$ and $|V| \geq 3$, the sets $U \cup g_{i}$ and $U \cup\left\{g_{i}, e\right\}$ are exact 3 -separations. In particular, $e \in \operatorname{cl}(V-e)$, so $r(V-e)>2$, since $e$ is not in a triangle. So $\left(U \cup g_{i},\{e\}, V-e\right)$ is a vertical 3 -separation, and hence $\operatorname{si}(M / e)$ is not 3-connected; a contradiction.

We deduce that $\operatorname{co}\left(M \backslash g_{i}\right)$ is 3-connected for each $i \in[t]-s$. As each $g_{i}$ is $N$-deletable, it is not contained in an $N$-grounded triad, so $M \backslash g_{i}$ is 3connected. We claim that there exists some $j \in[t]-s^{\prime}$ such that $L_{i} \cap K_{j}=\emptyset$. The claim follows immediately from 5.4.2, except when $P$ is an elongatedquad 3-separator. In such a case, suppose, without loss of generality, that $L_{1} \cup L_{2}=K_{1} \cup K_{2}$ is a quad; then $s, s^{\prime} \in\{1,2\}$, since $P-L_{s}$ is independent and $P-K_{s^{\prime}}$ is coindependent. Now $L_{3}=K_{3}$ is disjoint from $K_{j}$ for $j \in$ $\{1,2\}-s^{\prime}$, or $L_{i}$ for $i \in\{1,2\}-s$, satisfying the claim. After choosing such a $j$, 5.4.5 implies that there is an element $h_{j}$ such that $K_{j} \cup\left\{g_{i}, h_{j}\right\}$ is a cocircuit. Since the circuit $L_{i} \cup\left\{e, g_{i}\right\}$ meets the cocircuit $K_{j} \cup\left\{g_{i}, h_{j}\right\}$, it follows, by orthogonality, that $e=h_{j}$. Then $g_{i} \in \operatorname{cl}(P \cup e) \cap \mathrm{cl}^{*}(P \cup e)$ for each $i \in[t]-s$. If, say, $s \notin\{1,2\}$ and $g_{1} \neq g_{2}$, then $\lambda\left(P \cup\left\{e, g_{1}, g_{2}\right\}\right) \leq 1$, so $|E(M)-P| \leq 4$. Since the $N$-deletable element $g_{i}$ is not in a triad, and the $N$-contractible element $e$ is not in a triangle, $|E(M)-P|=4$, and 
$E(M)-P$ is a quad. Thus, if $P$ is not a double-quad 3 -separator or a spikelike 3-separator, then $|E(M)| \leq 10$. In the case that $P$ is a double-quad 3-separator, $|E(M)|=12$, and it is readily checked that $M$ is a quad-flower. In the case that $P$ is a spike-like 3 -separator, then $L_{i}=K_{i}$ for all $i \in[t]$, the set $L_{1} \cup\left\{e, g_{1}\right\}$ is a circuit, and $K_{3} \cup\left\{e, g_{1}\right\}$ and $K_{3} \cup\left\{e, g_{2}\right\}$ are cocircuits. But the latter two cocircuits imply $L_{3} \cup\left\{e, g_{1}, g_{2}\right\}$ is a 5-element coplane that intersects the circuit $L_{1} \cup\left\{e, g_{1}\right\}$ in two elements; a contradiction.

Now let $g_{i}=g_{j}=g$ for all $i, j \in[t]-s$. Suppose $P$ is a spike-like 3separator; then we may assume that $L_{i}=K_{i}$ for all $i \in[t]$, and there is no $s \in[t]$ for which $L_{s}$ has no element $N$-labelled for contraction. Then $L_{i} \cup\{e, g\}$ is a quad for all $i \in[t]$. Moreover, $e$ is $N$-labelled for contraction and, up to an $N$-label switch, $g$ is $N$-labelled for deletion. Therefore $P$ is not maximal; a contradiction.

Now $P$ is not a spike-like 3 -separator. We claim that $P \cup\{e, g\}$ is spanned by a $(t+1)$-element set. If $P$ is an elongated-quad 3 -separator or a twistedcube-like 3-separator of $M$, then $L_{s} \subseteq \operatorname{cl}\left(P-L_{s}\right)$, so by choosing an element from $L_{i}$ for each $i \in[t]-s$, together with $\{e, g\}$, we have such a set. Otherwise, $L_{i} \subseteq \operatorname{cl}\left(P-L_{i}\right)$ for any $i \in[t]$, so we take $\{e, g\}$ together with an element from $L_{i}$ for each $i \in[t-1]$. So $r(P \cup\{e, g\}) \leq t+1$. As $e \notin \operatorname{cl}(P)$, it now follows that $r(P) \leq t$; a contradiction.

5.4.7. No element in $E(M)-P$ is $N$-flexible.

Subproof. Suppose $x \in E(M)-P$ is $N$-flexible. Either $\operatorname{si}(M / x)$ or $\operatorname{co}(M \backslash x)$ is 3-connected, by Bixby's Lemma, which contradicts 5.4.6 or its dual. $\triangleleft$

5.4.8. No element in $E(M)-\operatorname{cl}(P)$ is $N$-labelled for contraction.

Subproof. Suppose there is an element $e \in E(M)-\operatorname{cl}(P)$ that is $N$-labelled for contraction. By 5.4.6, $\mathrm{si}(M / e)$ is not 3-connected. So $M$ has a vertical 3-separation $(U,\{e\}, V)$, with $|U \cap E(N)| \leq 1$, by Lemma 2.11. Without loss of generality, $V \cup e$ is closed. By the dual of Lemma 2.12, at most one element in $U$ is not $N$-flexible, and if such an element $x$ exists, then $x \in U \cap \mathrm{cl}^{*}(V)$ and $e \in \operatorname{cl}(U-x)$. By 5.4.7, we deduce that $U-x \subseteq P$ if such an $x$ exists, and $U \subseteq P$ otherwise. In either case, $e \in \operatorname{cl}(P)$; a contradiction.

5.4.9. There is at most one element in $\operatorname{cl}(P)-P$ that is $N$-labelled for contraction.

Subproof. Suppose $e, f \in \mathrm{cl}(P)-P$ are both $N$-labelled for contraction. If $r(E(M)-P) \geq 3$, then it follows that $(P \cup f,\{e\}, E(M)-(P \cup\{e, f\}))$ is a vertical 3-separation. By the dual of Lemma 2.12(ii), and as $f \in \operatorname{cl}(E(M)-$ $(P \cup\{e, f\}), f$ is also $N$-deletable, so $f$ is $N$-flexible. But this contradicts 5.4.7. So $r(E(M)-P) \leq 2$. Since $|E(M)-P| \geq 3$, the pair $\{e, f\}$ is contained in a triangle. As $M / e$ has an $N$-minor, it follows that $f$ is $N$ deletable, and hence $N$-flexible, contradicting 5.4.7.

By duality, 5.4 .8 and 5.4 .9 also imply the following: 
5.4.10. No element in $E(M)-\mathrm{cl}^{*}(P)$ is $N$-labelled for deletion, and there is at most one element in $\mathrm{cl}^{*}(P)-P$ that is $N$-labelled for deletion.

Finally, suppose that there exist distinct elements $g \in \operatorname{cl}(P)-P$ and $h \in \mathrm{cl}^{*}(P)-P$ such that $g$ is $N$-labelled for contraction and $h$ is $N$-labelled for deletion. Now $(P \cup g,\{h\}, E(M)-\{g, h\})$ is a cyclic 3-separation, with $g \notin \mathrm{cl}^{*}(E(M)-g)$, so $g$ is $N$-deletable by Lemma 2.12(i), contradicting 5.4.7. That completes the proof of the lemma.

\section{Proof of the THEOREM}

We first recall the main result from the first two papers in the series [4, Theorem 6.1].

Theorem 6.1. Let $M$ be a 3-connected matroid and let $N$ be a 3-connected minor of $M$ where $|E(N)| \geq 4$, and every triangle or triad of $M$ is $N$ grounded. Suppose, for some $d \in E(M)$, that $M \backslash d$ is 3-connected and has a cyclic 3-separation $\left(Y,\left\{d^{\prime}\right\}, Z\right)$ with $|Y| \geq 4$, where $M \backslash d \backslash d^{\prime}$ has an $N$-minor with $|Y \cap E(N)| \leq 1$. Then either

(i) $M$ has an $N$-detachable pair; or

(ii) there is a subset $X$ of $Y$ such that for some $c \in \mathrm{cl}_{M \backslash d}^{*}(X)-X$, one of the following holds:

(a) $X \cup\{c, d\}$ is a spike-like 3-separator of $M$,

(b) $X \cup\{c, d\}$ is a skew-whiff 3-separator of $M$,

(c) $X \cup\{c, d\}$ is a twisted-cube-like 3-separator of $M$ or $M^{*}$,

(d) $X \cup\{c, d\}$ is an elongated-quad 3-separator of $M$, or

(e) $X \cup\{a, b, c, d\}$ is a double-quad 3-separator of $M$ with associated partition $\{X,\{a, b, c, d\}\}$ for some distinct $a, b \in E(M)-(X \cup$ $\{c, d\})$.

We now prove the main result.

Theorem 6.2. Let $M$ be a 3-connected matroid with $|E(M)| \geq 11$, and let $N$ be a 3-connected minor of $M$ such that $|E(N)| \geq 4$, every triangle or triad of $M$ is $N$-grounded, and $|E(M)|-|E(N)| \geq 5$. Then either

(i) $M$ has an $N$-detachable pair;

(ii) there is some $P \subseteq E(M)$ such that $E(M)-E(N) \subseteq P$, and $P$ is

(a) a spike-like 3-separator,

(b) a skew-whiff 3-separator,

(c) a twisted-cube-like 3-separator of $M$ or $M^{*}$,

(d) an elongated-quad 3-separator,

(e) a double-quad 3-separator,

(f) a quad 3-separator,

or an augmentation of one of these particular 3-separators; or

(iii) $|E(M)|=12$, and $M$ is either a quad-flower or a nest of twisted cubes. 
Proof. By Lemma 3.3, if (i) does not hold, then, up to replacing $(M, N)$ by $\left(M^{*}, N^{*}\right)$, either

(I) there exists $d \in E(M)$ such that $M \backslash d$ is 3-connected and has a cyclic 3-separation $\left(Y,\left\{d^{\prime}\right\}, Z\right)$ with $|Y| \geq 4$, where $M \backslash d \backslash d^{\prime}$ has an $N$-minor with $|Y \cap E(N)| \leq 1$; or

(II) $M$ has an augmented quad 3-separator $Q \cup z$ such that $z \in \mathrm{cl}^{*}(Q)$ and $M \backslash z$ has an $N$-minor with $|Q \cap E(N)| \leq 1$; or

(III) there is an $N$-labelling $(C, D)$ of $M$ such that, for every switchingequivalent $N$-labelling $\left(C^{\prime}, D^{\prime}\right)$,

(a) $M / c$ and $M \backslash d$ are 3-connected for every $c \in C^{\prime}$ and $d \in D^{\prime}$,

(b) each pair $\left\{c_{1}, c_{2}\right\} \subseteq C^{\prime}$ is contained in a 4-element circuit, and

(c) each pair $\left\{d_{1}, d_{2}\right\} \subseteq D^{\prime}$ is contained in a 4-element cocircuit.

In case (III), if neither (ii) nor (iii) holds, then (I) or (II) holds by Proposition 4.1, Suppose (I) does not hold. Then (II) holds and, moreover, there is no element $e \in E(M)-(Q \cup z)$ such that $\{e, z\}$ is $N$-deletable and $M \backslash e$ is 3-connected, otherwise $(Q,\{z\}, E(M)-(Q \cup z))$ is a cyclic 3-separation satisfying (I), So, by an application of Lemma 5.1, (ii) holds.

Now we may assume that (I) holds. By Theorem 6.1, either (i) holds, or $Y$ contains a set $X$ such that, for some $c \in \mathrm{cl}_{M \backslash d}^{*}(X)-X$, either $X \cup\{c, d\}$ is a particular 3-separator $P$ that is a skew-whiff 3 -separator, a twisted-cube-like 3 -separator, or an elongated-quad 3-separator, or $X \cup\{c, d\}$ is contained in a double-quad 3 -separator $P$, or a maximal spike-like 3 -separator $P$. Thus, we can apply Lemma 5.4, and deduce that in the case that (i) does not hold, either $M$ is a quad-flower, so (iii) holds, or $E(M)-E(N) \subseteq P^{\prime}$ where $P^{\prime}=P$ or $P^{\prime}$ is an augmentation of $P$, so (ii) holds.

Using Lemma 2.15, we can relax the condition that every triangle or triad is $N$-grounded if we allow at most one $\Delta-Y$ or $Y-\Delta$ exchange.

Corollary 6.3. Let $M$ be a 3-connected matroid with $|E(M)| \geq 11$, and let $N$ be a 3 -connected minor of $M$ such that $|E(N)| \geq 4$ and $|E(M)|-|E(N)| \geq$ 5. Then either

(i) $M$ has an $N$-detachable pair;

(ii) there is a matroid $M^{\prime}$ obtained by performing a single $\Delta-Y$ or $Y-\Delta$ exchange on $M$ such that $M^{\prime}$ has an $N$-detachable pair; or

(iii) there is some $P \subseteq E(M)$ such that $E(M)-E(N) \subseteq P$, and $P$ is

(a) a spike-like 3 -separator,

(b) a skew-whiff 3-separator,

(c) a twisted-cube-like 3-separator of $M$ or $M^{*}$,

(d) an elongated-quad 3-separator,

(e) a double-quad 3-separator,

(f) a quad 3-separator, or an augmentation of one of these particular 3-separators; or

(iv) $|E(M)|=12$, and $M$ is either a quad-flower or a nest of twisted cubes. 
In particular, Corollary 6.3 implies Theorem 1.1.

\section{EPILOGUE}

Detachable pairs: the chain theorem. A spike is a matroid on $2 r$ elements, for some $r \geq 3$, with a partition $\left(L_{1}, L_{2}, \ldots, L_{r}\right)$ of the ground set into pairs such that $L_{i} \cup L_{j}$ is a quad for all distinct $i, j \in[r]$. Note that what we refer to as a spike is sometimes called a "tipless spike". A pair of elements $\left\{x_{1}, x_{2}\right\}$ in a matroid $M$ are detachable if either $M / x_{1} / x_{2}$ or $M \backslash x_{1} \backslash x_{2}$ is 3 -connected. Alan Williams proved the following chain theorem for detachable pairs in his Ph.D. thesis [12].

Theorem 7.1. Let $M$ be a 3-connected matroid with $|E(M)| \geq 13$. Then either

(i) $M$ has a detachable pair,

(ii) there is a matroid $M^{\prime}$ obtained by performing a single $\Delta-Y$ or $Y-\Delta$ exchange on $M$ such that $M^{\prime}$ has a detachable pair, or

(iii) $M$ is a spike.

His proof of this theorem takes a similar approach as the first two papers of this series [3, 4]; however, a number of the results in these two papers have simpler analogues when there is no $\mathrm{N}$-minor to worry about.

Alternatively, Theorem 7.1 can be obtained as a consequence of Theorem [1.1. Tutte's Wheels-and-Whirls Theorem [10] implies that every 3connected matroid with at least four elements has either $U_{2,4}$ or $M\left(K_{4}\right)$ as a minor. Thus, we can choose $N$ to be either $U_{2,4}$ or $M\left(K_{4}\right)$, in which case $|E(M)|-|E(N)| \geq 9$ or $|E(M)-| E(N) \mid \geq 7$ respectively, and then apply Theorem 1.1 and analyse the remaining structures. We omit the details.

$N$-detachable pairs in graphic matroids. We now consider the implications of Theorem 1.1 for graphic matroids. It is easy to check that the particular 3-separators in case (iii) of Theorem 1.1 cannot appear in graphic matroids. Hence, Theorem 1.1 has the following corollary:

Theorem 7.2. Let $M$ be a 3-connected graphic matroid, and let $N$ be a 3-connected minor of $M$ such that $|E(M)|-|E(N)| \geq 5$. Then either

(i) $M$ has an $N$-detachable pair, or

(ii) there is a matroid $M^{\prime}$ obtained by performing a single $\Delta-Y$ or $Y-\Delta$ exchange on $M$ such that $M^{\prime}$ has an $N$-detachable pair.

Let $G$ be a graph on at least four edges with no isolated vertices. It is well known that the cycle matroid $M(G)$ is 3-connected if and only if $G$ is 3connected and simple. Moreover, $\Delta-Y$ exchange in matroids generalises the corresponding operation in graphs, and a matroid obtained by performing a $\Delta-Y$ exchange on a triangle of $M(G)$ is graphic. However, graphic matroids are not closed under $Y-\Delta$ exchange; if $M(G)$ is a graphic matroid, and $M^{\prime}$ is obtained from $M(G)$ by a $Y$ - $\Delta$ exchange on a triad $T^{*}$, then $M^{\prime}$ is graphic if and only if $T^{*}$ is not a separating triad. Nevertheless, we expect that 
a careful analysis of Lemma 2.15 for graphic matroids would confirm the following conjecture:

Conjecture 7.3. Let $G$ be a simple 3-connected graph, and let $H$ be a simple 3 -connected minor of $G$ such that $|E(G)|-|E(H)| \geq 5$. Then either

(i) $G$ has a pair of edges $\{x, y\}$ such that either $G / x / y$ or $G \backslash x \backslash y$ is simple, 3-connected, and has an isomorphic copy of $H$ as a minor, or

(ii) there is a graph $G^{\prime}$ obtained by performing a single $\Delta-Y$ or $Y-\Delta$ exchange on $G$ such that $G^{\prime}$ has a pair of edges $\{x, y\}$ for which either $G^{\prime} / x / y$ or $G^{\prime} \backslash x \backslash y$ is simple, 3-connected, and has an isomorphic copy of $H$ as a minor.

Similarly, we conjecture the following analogue of Theorem 7.1 .

Conjecture 7.4. Let $G$ be a simple 3-connected graph such that $|E(G)| \geq$ 10. Then either

(i) $G$ has a pair of edges $\{x, y\}$ such that either $G / x / y$ or $G \backslash x \backslash y$ is a simple 3-connected graph, or

(ii) there is a graph $G^{\prime}$ obtained by performing a single $\Delta-Y$ or $Y-\Delta$ exchange on $G$ such that $G^{\prime}$ has a pair of edges $\{x, y\}$ for which either $G^{\prime} / x / y$ or $G^{\prime} \backslash x \backslash y$ is a simple 3-connected graph.

Eliminating $\Delta-Y$ s. A natural question is what additional structures arise if no $\Delta-Y$ or $Y-\Delta$ exchange is permitted.

As a starting point, we consider here an analogue of Tutte's Wheels-andWhirls Theorem; that is, an analogue of Theorem 7.1 where case (ii) is not allowed. Certainly, if $M$ is a wheel or a whirl, then it follows from Tutte's Wheels-and-Whirls Theorem that $M$ has no detachable pairs. It is not hard to see that $M\left(K_{3, k}\right)$ and, dually, $M^{*}\left(K_{3, k}\right)$ also have no detachable pairs, for any $k \geq 3$.

A spike has a unique single-element extension by an element that is in the closure of each of the legs, and a unique single-element coextension by an element that is in the coclosure of each of the legs; we call a matroid with both a spike with tip and cotip. If $M$ is a spike with tip and cotip, then $M$ has no detachable pairs. In fact, these matroids are examples of a more general family of matroids with no detachable pairs, which we now describe.

Let $M$ be a spike with tip $t$ and cotip $s$, whose $q$ legs are the pairs $\left\{x_{i}, y_{i}\right\}$ for $i \in[q]$, where $q \geq 2$. Note that $M$ has rank $q+1$, and we consider the rank-3 matroid $Q_{6}$ to be the unique tipped and cotipped spike with two legs. Let $0 \leq p \leq q$. For each $i \in[p]$, attach a wheel of rank at least three along the triangle $\left\{x_{i}, y_{i}, t\right\}$, where $t$ and $y_{i}$ are spokes of the wheel, by generalised parallel connection. Finally, delete $x_{i}$ for each $i \in[p]$. We say that such a matroid is constructed by attaching wheels to a spike with tip and cotip. Observe in particular that $M$ consists of $q$ maximal fans, each of even size and with ends $t$ and $s$ (see [3, Section 2] for the definition, and connectivity 
properties, of fans). It follows that any such matroid $M$ has no detachable pairs.

Finally, let $M$ be a paddle $\left(P_{1}, \ldots, P_{n}\right)$, for $n \geq 3$, with spine $\{s, t\}$ (that is, $\operatorname{cl}\left(P_{1}\right) \cap \cdots \cap \operatorname{cl}\left(P_{n}\right)=\{s, t\}$, see [7]) where for each $i \in[n]$, the set $P_{i} \cup\{s, t\}$ is an odd maximal fan with ends $s$ and $t$. Such a matroid can be constructed as follows. Let $L$ be a line containing the pair $\{s, t\}$, and consisting of at least three elements. Attach at least three wheels, each of rank at least three, and each along some triangle containing $\{s, t\}$, by generalized parallel connection. Finally, delete $L-\{s, t\}$. We say that such a matroid can be constructed by attaching wheels with common spokes $s$ and $t$. If we also delete $s$, we obtain a matroid with no detachable pairs. The simplest example of such a matroid is $M\left(K_{3, n}^{\prime}\right)$, where $K_{3, n}^{\prime}$ is obtained from $K_{3, n}$ by adding a single edge between vertices in the part of size three.

We conjecture the following:

Conjecture 7.5. Let $M$ be a 3-connected matroid with $|E(M)| \geq 13$. Then either

(i) $M$ has a detachable pair,

(ii) $M$ is a spike,

(iii) $M \cong M\left(K_{3, k}\right)$ or $M \cong M^{*}\left(K_{3, k}\right)$ for some $k \geq 5$,

(iv) $M$ is the cycle matroid of a wheel, or a whirl,

(v) $M$ can be constructed by attaching wheels to a spike with tip and cotip, or

(vi) $M$ or $M^{*}$ can be constructed by attaching wheels with common spokes $s$ and $t$, and then deleting $s$.

We note that the matroids in (v), and matroids similar to those in (vi), appear in the work of Oxley and Wu on matroids with exactly two nonessential elements [8]. An element $e \in E(M)$ is essential if neither $M \backslash e$ nor $M / e$ is 3-connected. Oxley and $\mathrm{Wu}$ showed that the class of 3-connected matroids with precisely two non-essential elements, one of which must be deleted and one of which must be contracted, is the class of matroids that can be constructed by attaching wheels to a spike with tip and cotip [8, Theorem 1.4]. Meanwhile, the class of 3-connected matroids with precisely two non-essential elements, both of which must be deleted, are matroids that can be constructed by attaching wheels with common spokes $[8$, Theorem 1.3]. A matroid of case (vi) is obtained by deleting a further element from the spine of the paddle.

We now focus on simple 3-connected graphs. By interpreting Conjecture 7.5 for graphic matroids, we obtain a conjecture regarding the existence of "detachable pairs" in simple 3-connected graphs.

We first require some definitions; these follow 8. Consider a copy of $K_{4}$ where the edges $e$ and $f$ are non-adjacent, and let $u$ and $v$ be vertices such that $u$ incident to $f$, and $v$ is incident to $e$. A twisted wheel is a graph that can be obtained by subdividing $e$ so that $j \geq 0$ new vertices are introduced, 
adding $j$ edges between each of these vertices and $u$; then subdividing $f$ so that $k \geq 0$ new vertices are introduced, and adding $k$ edges between each of the $k$ new vertices and $v$.

A multi-dimensional wheel is constructed as follows: begin with the 3vertex path $u h v$, and add $k \geq 3$ parallel edges between $u$ and $v$. For each of the $k$ parallel edges, subdivide it so that one or more new vertices are introduced, and join each new vertex to $h$. A graph obtained by then removing the edge $u h$ is an unhinged multi-dimensional wheel.

We conjecture the following:

Conjecture 7.6. Let $G$ be a simple 3-connected graph with $|E(G)| \geq 13$. Then either

(i) $G$ has a pair of edges $\{x, y\}$ such that either $G \backslash x \backslash y$ or $G / x / y$ is a simple 3-connected graph,

(ii) $G$ is a wheel,

(iii) $G \cong K_{3, k}$ for some $k \geq 5$,

(iv) $G$ is a twisted wheel, or

(v) $G$ is an unhinged multi-dimensional wheel.

\section{REFERENCES}

[1] R. E. Bixby. A simple theorem on 3-connectivity. Linear Algebra and its Applications, 45:123-126, 1982.

[2] N. Brettell and C. Semple. A Splitter Theorem relative to a fixed basis. Annals of Combinatorics, 18(1):1-20, 2014.

[3] N. Brettell, G. Whittle, and A. Williams. $N$-detachable pairs in 3-connected matroids I: unveiling X. Journal of Combinatorial Theory, Series B, 141:295-342, 2020.

[4] N. Brettell, G. Whittle, and A. Williams. N-detachable pairs in 3-connected matroids II: life in X. Journal of Combinatorial Theory, Series B, 2020.

[5] B. Clark. Fragility and excluded minors. Ph.D. thesis, Victoria University of Wellington, 2015.

[6] J. Oxley. Matroid Theory, volume 21 of Oxford Graduate Texts in Mathematics. Oxford University Press, New York, second edition, 2011.

[7] J. Oxley, C. Semple, and G. Whittle. The structure of the 3-separations of 3-connected matroids. Journal of Combinatorial Theory, Series B, 92(2):257-293, 2004.

[8] J. Oxley and H. Wu. Matroids and graphs with few non-essential elements. Graphs and Combinatorics, 16(2):199-229, 2000.

[9] P. D. Seymour. Decomposition of regular matroids. Journal of Combinatorial Theory, Series B, 28(3):305-359, 1980.

[10] W. T. Tutte. Connectivity in matroids. Canadian Journal of Mathematics, 18:13011324, 1966.

[11] G. Whittle. Stabilizers of classes of representable matroids. Journal of Combinatorial Theory, Series B, 77(1):39-72, 1999.

[12] A. Williams. Detachable Pairs in 3-Connected Matroids. Ph.D. thesis, Victoria University of Wellington, 2015.

School of Mathematics and Statistics, Victoria University of Wellington, New Zealand

Email address: nbrettell@gmail.com

Email address: geoff.whittle@vuw.ac.nz

Email address: ayedwilliams@gmail.com 